\title{
Metallurgy Division Fy 2004 Programs and Accomplishments
}
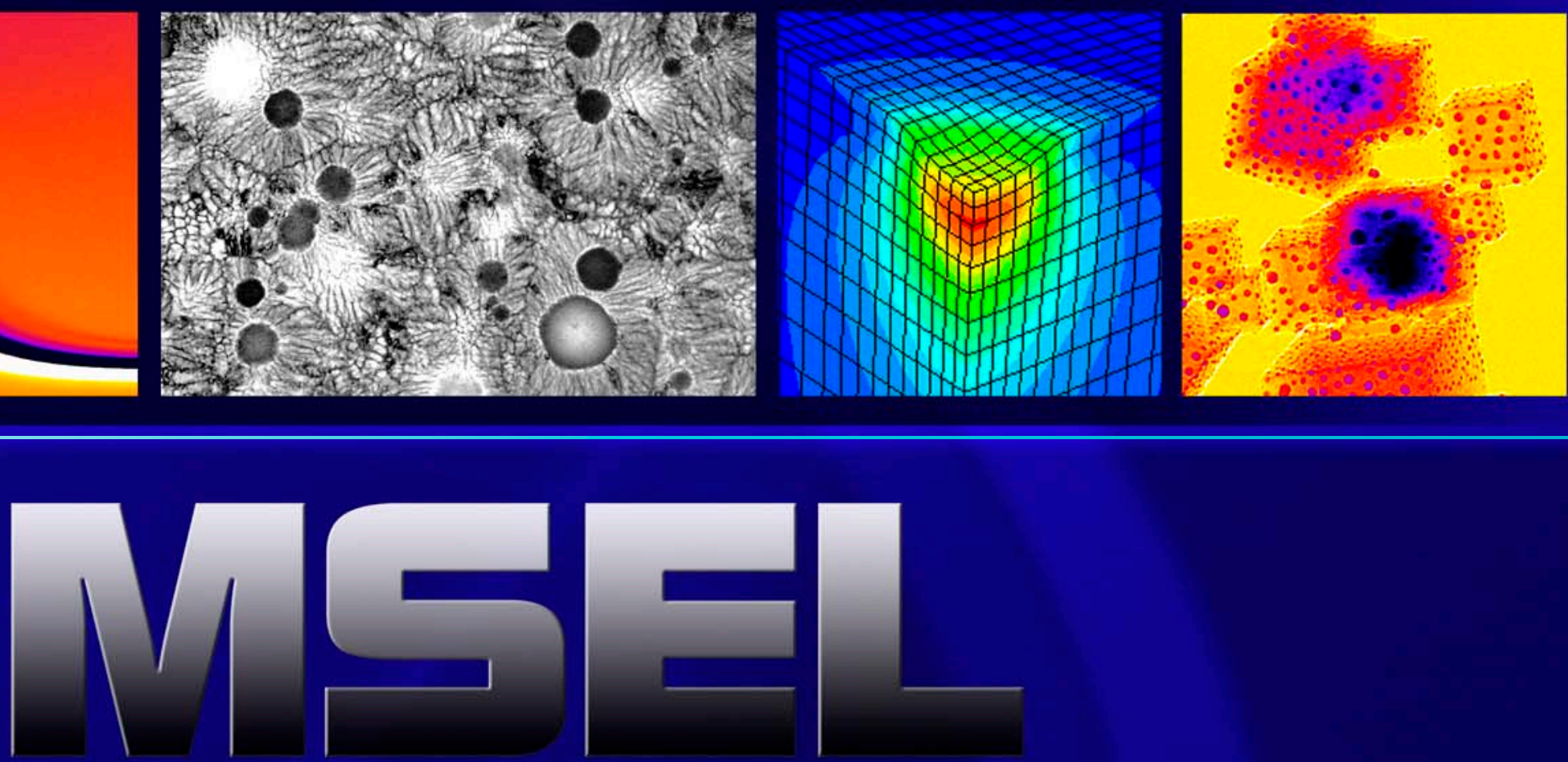

Materials Science and Engineering Laboratory

\section{NGT}

National Institute of Standards and Technology

Technology Administration

U.S. Department of Commerce

NISTIR 7127

September 2004 


\section{On the Cover:}

Fig. 1 - (on left, on back cover) Ferromagnetic resonance measurements of a Permalloy film on a specially roughened copper substrate as a function of applied field direction. These measurements are part of a study to determine the effects of roughness on magnetization dynamics.

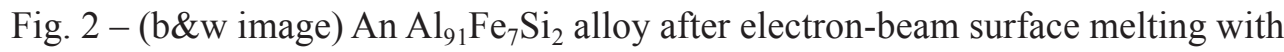
scan velocity of $50 \mathrm{~cm} / \mathrm{sec}$ (transmission electron micrograph). This observation of a glassy phase which has characteristics of a first-order transformation (as do normal crystalline phases) holds promise for producing stable metallic glass phases.

Fig. 3 - FEM model of a rigid $100 \mathrm{~nm}$ diameter sphere indenting an Al sample to a depth of $10 \mathrm{~nm}$. The FEM mesh is fine enough to use the predicted elastic displacement fields to generate boundary conditions and initial atom positions for an atomistic simulation using classical potentials.

Fig. 4 - (near edge of front cover) $\mathrm{MgO}$ cubes with surfaces decorated by small gold particles. These particle ensembles are used in model studies of 3D chemical imaging at the nanoscale (electron tomography). 
National Institute of

Standards and Technology

Arden L. Bement, Jr.

Director

Technology

Administration

Phillip J. Bond

Undersecretary of

Commerce for Technology

U.S. Department

of Commerce

Donald L. Evans

Secretary

\section{Materials Science and Engineering Laboratory}

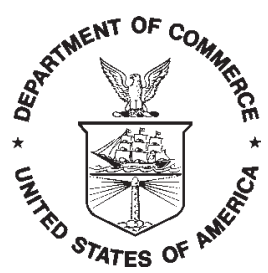

\section{FY 2004 Programs and ACCOMPlishmENTS}

\section{Metallurgy \\ Division}

Carol A. Handwerker, Chief

Frank W. Gayle, Deputy

NISTIR 7127

September 2004 
Certain commercial entities, equipment, or materials may be identified in this document in order to describe an experimental procedure or concept adequately. Such identification is not intended to imply recommendation or endorsement by the National Institute of Standards and Technology, nor is it intended to imply that the entities, materials, or equipment are necessarily the best available for the purpose. 


\section{Table of Contents}

Executive Summary 1

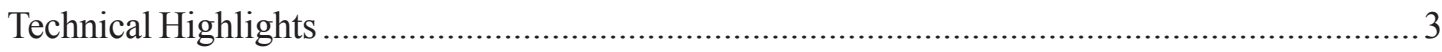

Formation of Glass by a First Order Transition ............................................................ 4

Interactions with Industry: Delivering Data and

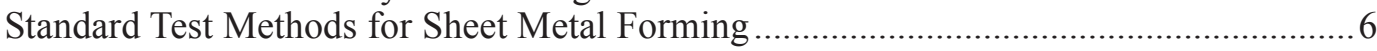

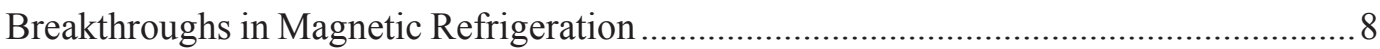

Grand Challenges in Nanomagnetics: High Coercivity FePt

Alloys for Future Perpendicular Magnetic Data Storage

On-Chip Interconnects:

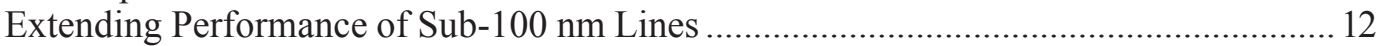

The Structural Steel of the World Trade Center Towers ..................................................... 14

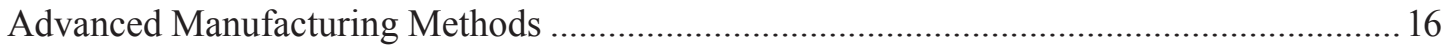

Sheet Metal Forming for Automotive Applications:

Anelasticity and Springback Prediction

Microstructural Origins of Surface Roughening

and Strain Localizations 18

Plasticity, Fabrication Processes, and Performance ……….............................................. 19

Standard Tests and Data for Sheet Metal Formability .....................................................2 20

The NIST Center for Theoretical and Computational

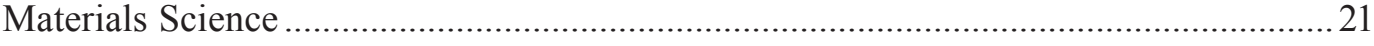

Underlying Processes of Plastic Deformation in Metal Alloys ........................................22

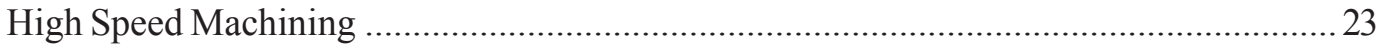

Mechanisms for Delivery of Thermodynamic and Kinetic Data ....................................24

Phase Field Modeling of Materials Systems:

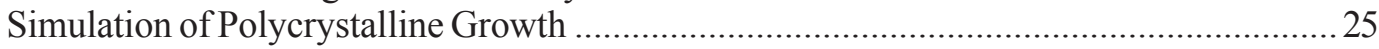

Hardness Standardization: Rockwell, Vickers, Knoop ....................................................26

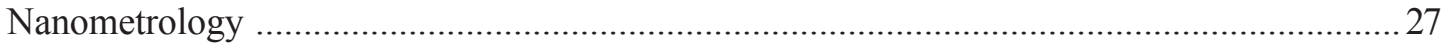

Nanoscale Characterization by Electron Microscopy ……..............................................2 28

Nanostructure Fabrication Processes:

Patterned Electrodeposition by Surfactant-Mediated Growth ..........................................2 29

Nanostructure Fabrication Processes:

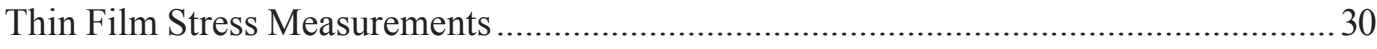

Nanomechanics: Coupling Modeling with Experiments ................................................... 31 


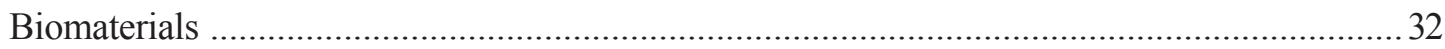

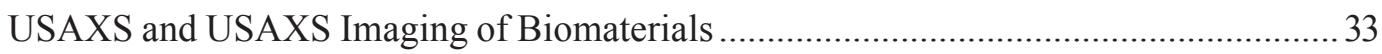

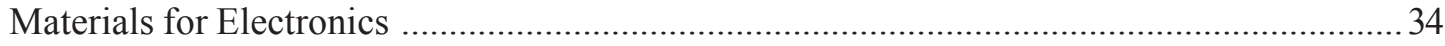

Combinatorial/Phase Diagram Approach for

Metallization to Wide-Band-Gap Semiconductors .............................................................. 35

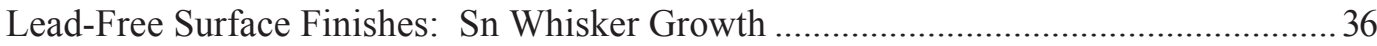

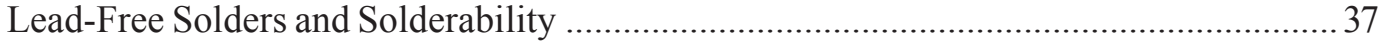

Electrical Properties of On-Chip Interconnections ............................................................ 38

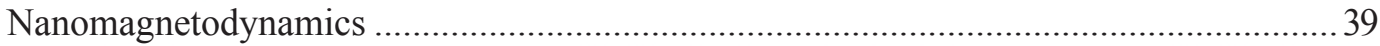

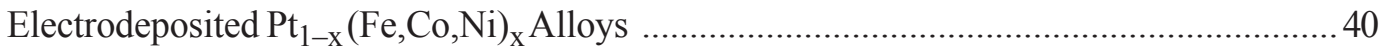

Novel Magnetic Materials for Sensors and

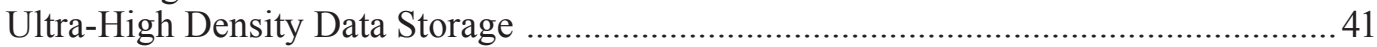

Discovery of Spin Density Waves in a Ferromagnet: Fe-Al ......................................... 42

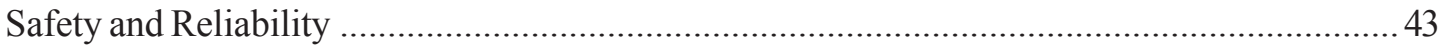

Analysis of Structural Steel in the World Trade Center ...................................................... 44

Standard Test Methods for Fire-Resistant Steel ............................................................. 45

Pipeline Safety: Corrosion, Fracture and Fatigue ………................................................. 46

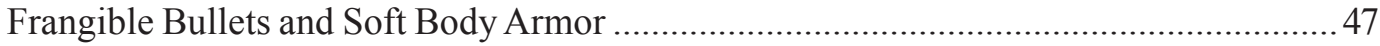

Marine Forensics and Preservation of Historic Shipwrecks ........................................... 48

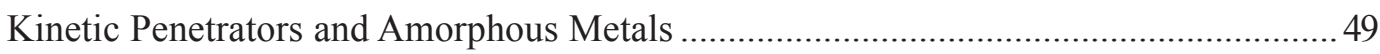

Metallurgy Division FY04 Annual Report Publication List ................................................. 51

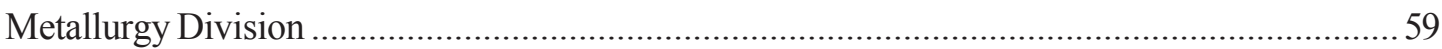

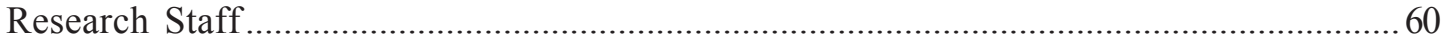

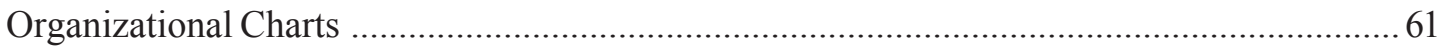




\section{Executive Summary}

This report describes the major technical activities and accomplishments in the Metallurgy Division of NIST Materials Science and Engineering Laboratory in FY 2004 (October 2003 through September 2004). In this report, we have tried to provide insight into how the capabilities of the NIST Metallurgy Division are being used to solve problems important to the national economy and the materials metrology infrastructure, and how we establish new programs in response to changes in national priorities. We welcome feedback and suggestions on how we can better serve the Nation and encourage increased collaboration to this end.

\section{Mission}

Our mission is to provide critical leadership in the development of measurement methods, standards, and fundamental understanding of materials behavior needed by U.S. materials users and producers to become or remain competitive in the changing global marketplace. As a fundamental part of this mission, we are responsible not only for developing new measurement methods with broad applicability across materials classes and industries, but also for working with other federal agencies and industrial sectors to develop and integrate measurements, standards, software tools, and evaluated data for specific, important applications.

\section{Establishing Priorities}

We examine a wide range of research opportunities and set priorities based on the following criteria: the match to the NIST mission, the magnitude and immediacy of industrial need, the determination that the NIST contribution is critical for success, the anticipated impact relative to our investment, the ability to respond in a timely fashion with high-quality output, and the opportunity to advance mission science. We make such decisions using a variety of methods, including roadmapping activities, workshops, technical meetings, standards committee participation, and consultation with individuals representing U.S. industry and other federal agencies.

Technology trends strongly influence the technical directions addressed by NIST. We prefer to work in rapidly evolving technologies, where advances in measurement science are needed to understand the limitations on system behavior, and, thus, our contributions are likely to have a substantial impact on the course of technology. For NIST as a whole and the Metallurgy Division in particular, we are committed to having an impact on nanotechnology, homeland security, health care, and information technology.
Over the last two years, we have shifted substantial resources into the areas of nanomagnetics, nanomechanics, and the application of thermodynamics and kinetics to nanostructure fabrication, and into a new MSEL Program in Safety and Reliability of Infrastructure Materials with a focus on homeland security and critical infrastructure protection. The Division highlights and the individual project descriptions demonstrate our rapid progress in these and other longer-standing areas.

\section{Research Portfolio}

Our 2004 research portfolio focuses on fulfilling current and future measurement needs of the magnetic data storage, microelectronics packaging, automotive, optoelectronics, and energy distribution industries, on establishing national traceable hardness standards needed for international trade, and on developing new measurements that could underpin nanotechnology development. Our output consists of a variety of forms, from a fundamental understanding of materials behavior to measurement techniques conveyed through the scientific literature and oral presentations, standard reference materials, evaluated data and online databases, software tools, and sensors for on-line process control.

\section{Division Structure and Expertise}

The Division is composed of 34 scientists, supported by 5 technicians, 6 administrative staff members, and more than 80 guest scientists, and it is organized into five groups that represent the Division's core expertise in Metallurgical Processing, Electrochemical Processing, Magnetic Materials, Materials Structure and Characterization, and Materials Performance. However, by virtue of the interdisciplinary nature of materials problems in the industrial and metrology sectors that we serve, Program teams are assembled across group, division and laboratory boundaries and with external partners to best meet project goals. We are committed to assembling the expertise and resources to fulfill our technical goals with the speed and quality necessary to have the desired impact.

\section{Recognition for Division Staff}

We are proud of the accomplishments of the Metallurgy Division staff in delivering the mission science tools - measurements, standards, data, modeling — needed by our customers. In FY2004, Division members were recognized for the impact and quality of their work by a wide range of organizations, 
including the award of two Gold Medals, the highest honor conferred by the Department of Commerce, and one Silver Medal from the Department of Commerce and NIST.

- Bill Egelhoff was awarded a 2003 Department of Commerce Gold Medal for his world leadership in the science and engineering of magnetic thin films. His contributions to the understanding of magnetism, electron transport and thin film deposition have been made possible by his Magnetic Engineering Research Facility, which has enabled him to measure and control the deposition processing conditions better than anyone else in the world. Bill has earned an international reputation as a leader in this field and has produced significant economic benefits to the U.S. magnetic data storage industry.

- Carol Handwerker, Ursula Kattner, Bill Boettinger, Maureen Williams, Frank Gayle, Frank Biancaniello, and Tom Siewert were awarded the Department of Commerce Gold Medal for their scientific and technical leadership in providing the microelectronics industry with the critical measurements, modeling, and data necessary for the successful world-wide conversion to lead-free solder in microelectronics manufacturing.
- Richard Fields was awarded the Department of Commerce Silver Medal for his important contributions to the science of materials property measurements and modeling. His work has resulted in the recognition of NIST as an important source of expertise and leadership by other government agencies and the industrial sector when materials performance questions need solutions.

- Ursula Kattner was named a Fellow of ASM International for her outstanding achievement in thermodynamics and the application of phase diagrams to important industrial metallurgical processes.

- Bob McMichael was named an IEEE Magnetics Society Distinguished Lecturer for 2004.

\author{
Carol A. Handwerker \\ Chief, Metallurgy Division
}




\section{Technical Highlights}

The following Technical Highlights section includes expanded descriptions of research projects that have broad applicability and impact. These projects generally continue for several years. The results are the product of the efforts of several individuals. The Technical Highlights include:

- Formation of Glass by a First Order Transition

- Interactions With Industry: Delivering Data and Standard Test Methods for Sheet Metal Forming

- Breakthroughs in Magnetic Refrigeration

- Grand Challenges in Nanomagnetics: High Coercivity FePt Alloys for Future Perpendicular Magnetic Data Storage

- On-Chip Interconnects: Extending Performance of Sub-100 nm Lines

- The Structural Steel of the World Trade Center Towers 


\section{Formation of Glass by a First Order Transition}

\begin{abstract}
Recently the aerospace, military and electronics industries have devoted much effort to the development of practical materials that are $100 \%$ bulk metallic glass but do not require extremely rapid quenching. Most glassy solids are thought to be kinetically frozen liquids, thus the usual approach is to cool fast enough to prevent formation of crystals. We have presented evidence that an isotropic non-crystalline metallic phase (dubbed "q-glass") in the Al-Fe-Si system forms during rapid cooling of the melt by a first order transition, like a crystalline phase, suggesting the possibility of low-energy, non-periodic structures and the formation of glasses by relatively slow growth.
\end{abstract}

\section{Leonid A. Bendersky and John W. Cahn}

$M$ uch DoD research has focused recently on the development of bulk metallic glasses (e.g., DARPA SAM program) for use as both armor materials and kinetic penetrators. Production of such glasses requires moderate rates of melt quenching in contrast to the rapid quenching required for conventional metallic glasses. These studies have all assumed that the glassy phases form only as a frozen liquid. In order to gain an understanding of the processing routes available to control these and related materials, it is essential to determine how these structures actually form. Such distinctions in the formation of various glassy phases may play an important role in their relative ductility, corrosion resistance and stability. The compositional and structural nature of the glassy phases is, thus, a question of more general interest.

\section{An Unusual Microstructure Found in Rapidly Solidifed Al-Fe-Si}

Recently we presented evidence that an isotropic non-crystalline metallic phase (dubbed "q-glass") could be formed by growth from a melt. This phase is the first phase, or "primary phase," to form in the Al-Fe-Si system during rapid cooling of the melt. ${ }^{[1]}$ There is strong evidence that this phase forms from the melt by a first order transition. According to transmission electron microscope (TEM) observations, Figure 1a, the q-glass nucleates from the melt as isolated particles, which initially grow as spheres (nodules). The diffraction pattern taken from a nodule shows it to be an isotropic glassy phase, Figure 1c. Dark field,
Figure $1 \mathrm{~b}$ and high-resolution TEM imaging, Figure 2, also support the isotropic, but highly speckled, nature of the phase. There is a nucleation barrier, which implies an interface between the glass and the melt. Further evidence for the first order transition is found in the solute partitioning at this interface; the q-glass rejects $\mathrm{Al}$ into the melt, which ultimately solidifies as a eutectic composed mainly of crystalline fcc aluminum and more q-glass. Presumably glass and melt are distinct phases with an interface having a positive, surface-free energy. Such solidification microstructures are familiar and well understood, except that here the glass is the first phase growing from the melt and eventually becomes surrounded by eutectic, instead of being the last phase (sometimes the only phase) formed when atomic motion ceases in the melt.
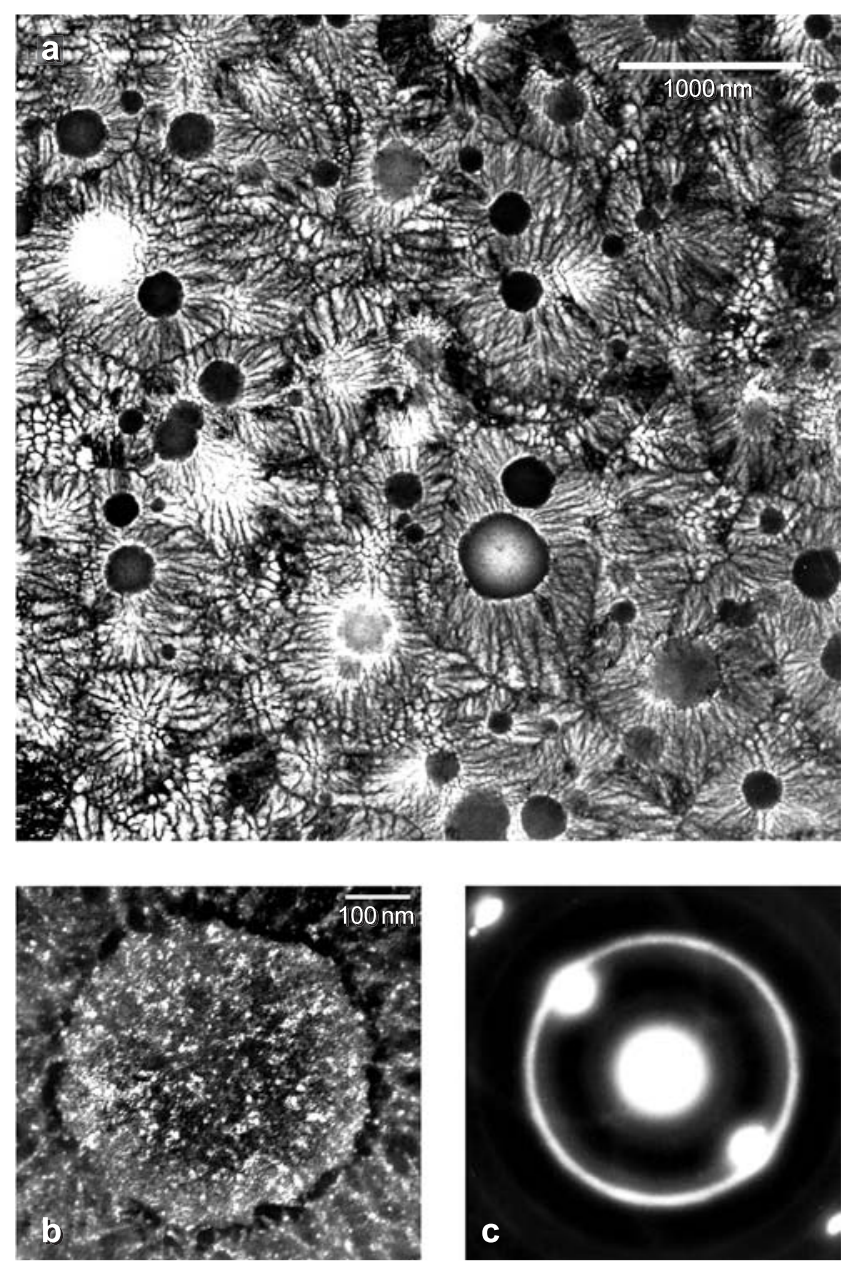

Figure 1: a) Microstructure of an $\mathrm{Al}_{91} \mathrm{Fe}_{7} \mathrm{Si}_{2}$ alloy after electron-beam surface melting with scan velocity of $50 \mathrm{~cm} / \mathrm{sec}$. b) Dark field image of a nodule and the glassy phase in the monotectic, obtained by positioning a SAD aperture on the strongest diffuse ring in c). 


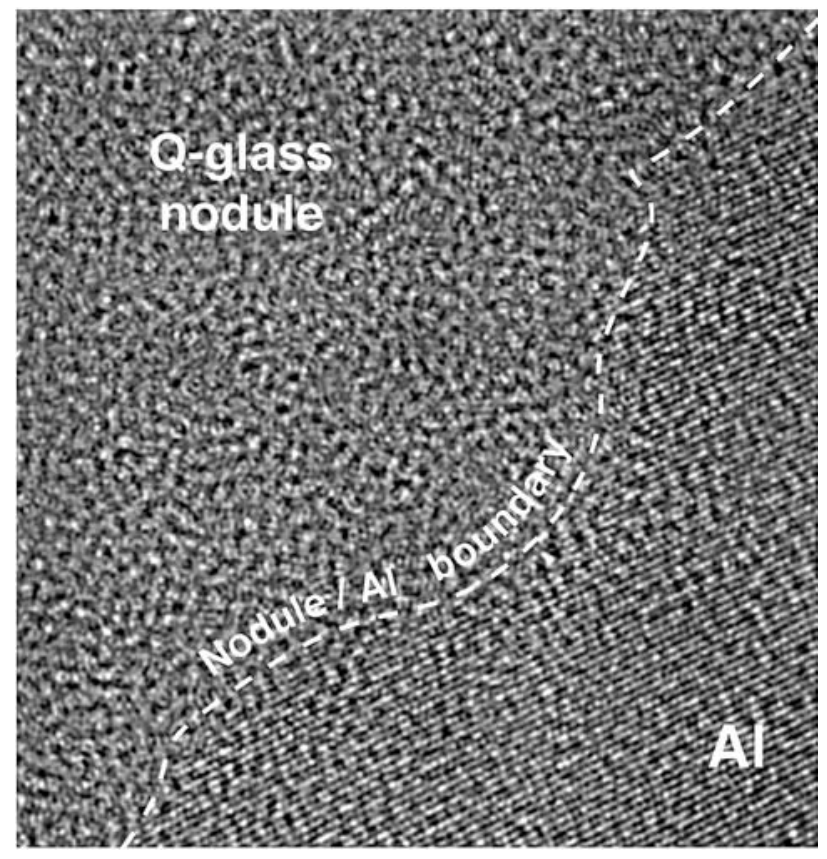

Figure 2: High-resolution TEM image showing an interface between a glassy nodule (q-glass) and Al. Note the isotropic phase contrast of the q-glass.

\section{Q-Glass Properties}

Stability. A lower entropy implies a higher degree of order, which in turn is consistent with a process of growth that selects some components and rejects others. This seems to be the case for the q-glass where $\mathrm{Al}$ is rejected into the melt. The lower entropy implies that at low temperatures the q-glass may have greater stability than a conventional glass (frozen liquid) of the same composition, and that there would be a latent heat associated with its formation which, as is true for all incongruent freezing, is spread over a freezing range.

The q-glass in $\mathrm{Al}-\mathrm{Fe}-\mathrm{Si}$ is metastable, as were the original AlMn quasicrystals. Based on the quasicrystal experience, it is expected that, in time, fully stable q-glasses will be found in systems with more components.

Structure and Order. The implied order and narrower composition range are closely related. We expect q-glasses to have a chemical order with a smaller set of local environments. Recently, there have been many advances in creating orderly arrangements which are not crystalline.[2] This work suggests that it might be possible that a glassy structure is composed of unit cells which are not parallelepipeds and cannot be packed in a periodic or quasiperiodic way. Unlike the pinwheel tilings in two dimensions which take on every orientation but need matching rules to prevent them from packing periodically, there are 3-D Schmitt-Conway-Danzig tiles which can fill space only in an aperiodic way. ${ }^{[2]}$ If atoms pack into such a unit cell, q-glasses could be stoichiometric and have low entropy, as do crystalline compounds.

There is some confirmation for such order in hydrogen-storage alloy glasses. For these glasses, the charging and discharging pressure have a long plateau, just as for crystals. This unusual behavior for glasses implies a highly ordered glass structure in which there is only one type of site for the stored hydrogen.

\section{On-Going Work}

The concept of q-glass was introduced at the MRS meeting last year at "Symposium MM: Amorphous and Nanocrystalline Metals."'[1] Since then, our work has received support from the Air Force Office of Scientific Research. Current work is focused on three areas:

1. Metastable Al-Fe-Si phase diagram. The ternary Al-Fe-Si phase diagram has been extensively studied. Thermodynamic principles of metastable phase equilibria are well developed, and much is already known about metastable crystalline phases in this ternary system, but not about the q-glass. We are investigating conditions for q-glass formation and its subsequent conversion to other phases to map out the bounds of q-glass on the metastable phase diagram. In addition, calorimetric studies are being conducted to evaluate the thermodynamics of q-glass formation.

2. Other glass forming Al-based systems. Judging from the current literature, it is possible that the q-glass forms in other Al-based systems. ${ }^{[3]}$ We use morphological criterion to distinguish the formation of q-glass from conventional glass, with a special focus on systems known for forming metallic glasses.

3. Other methods of growing q-glass. Feasibility studies to fabricate and study the formation and stability of metallic glasses will be conducted using combinatorial methods. Libraries of amorphous metal thin films will be fabricated using the new Metallurgy Division e-beam evaporation system by depositing elemental wedge-shaped layers controlled by moving shutters. This combinatorial approach will be applied to measuring the stability and control of glasses.

\section{For More Information on this Topic}

1. J.W. Cahn and L.A. Bendersky, "An isotropic glass phase in Al-Fe-Si formed by a first order transition," MRS 2003 Proceedings.

2. M. Senechal, Quasicrystals and Geometry, New York: Cambridge University Press, Chap. 7, (1995).

3. A. Inoue, M. Watanabe, H.M. Kimura, A. Nagata, and T. Masumoto, Matls. Trans., 8, 723 (1992). 


\section{Interactions with Industry: Delivering Data and Standard Test Methods for Sheet Metal Forming}

\begin{abstract}
The automotive industry's push to incorporate lighter sheet metal parts into new car designs has been hampered by lack of detailed data on mechanical behavior for forming. The Metallurgy Division has developed new metrology and test methods to address specific problems identified by industry as inhibiting broad adaptation of high-strength steel and aluminum sheet in automotive applications.
\end{abstract}

\section{Tim Foecke, Mark Stoudt, and Mark ladicola}

$\mathrm{T}$ The Sheet Metal Forming program within the Metallurgy Division has been designed from the outset to be relevant to the American manufacturing sector. Surveys conducted by NIST staff through the Industrial Liaison Office (ILO) sought to determine which aspects of sheet metal forming were most in need of better data and metrology. The results revealed that industry had a clear understanding of how NIST expertise could be applied and predicted a high potential impact if the research was successfully implemented.

The U.S. automotive industry estimates that the costs associated with the inability of the finite element models to adequately predict friction, multiaxial hardening, or springback are on the order of hundreds of millions of dollars annually. These shortcomings are the direct result of insufficient property data and the physical understanding that such data can reveal. The projects described here are specifically designed to address the quality of the sheet metal property data and models used to develop predictive finite element models of forming operations.

\section{The Microstructural Origin of Roughening and Strain Localization}

Surface roughness is a major contributor to friction during sheet metal forming, and thus must be well controlled in order to obtain a consistent product. Furthermore, the surface qualities of the finished product determine ultimate aesthetic characteristics of the vehicle. Ability to accurately characterize surface roughness is, therefore, of critical importance to the industry.

There are many sources of error in surface roughness measurements. Recent studies of the discrepancies between measured and predicted surface roughness show that the greatest error is introduced by the numerical tools used routinely by industry to interpret the roughness data. Parameters such as the rms roughness $(\mathrm{Rq})$ compress complex surface details into singular expressions that do not adequately represent the surface features involved (Figure 1). Changes in the surface feature arrangement, which are not captured by the rms roughness, have a greater influence on friction. Hence, any prediction of friction that is based solely on a tool such as the rms roughness is likely to yield erroneous results.

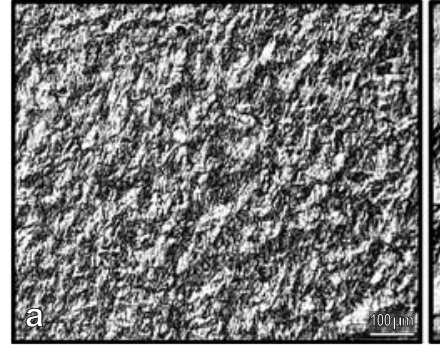

$\mathrm{Rq}=0.142 \mu \mathrm{m} \pm 0.023 \mu \mathrm{m}$

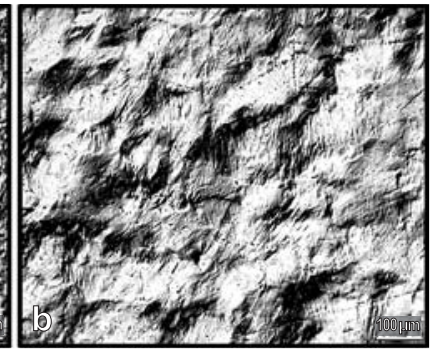

$\mathrm{Rq}=0.144 \mu \mathrm{m} \pm 0.007 \mu \mathrm{m}$
Figure 1: Two surface conditions exhibiting the same roughness mathematically, but very different appearances and frictional behaviors.

These results were presented to the automotive industry at several major research conferences in the past year. Invited presentations included talks at the "2004 Society of Automotive Engineers World Congress" in Detroit, MI, the "2004 North American Deep Drawing Research Group" spring meeting at NIST, the "Springback Compensation Program" and the "Agile Flexible Binder Control" quarterly meetings held at the U.S. Council for Automotive Research (USCAR) in Southfield, MI, as well as the "Automotive Sheet Steels Symposium" in Chicago, IL. The results were also published in both archival journal and in conference proceeding formats.

Overall, the industrial response has been very positive, and there is a high level of interest in the outcome of new studies. The high-resolution surface topography data obtained with the Metallurgy Division's scanning laser confocal microscope has been particularly well received and has stimulated several new opportunities identified by our industrial contacts for direct collaboration with the automotive industry.

\section{Multiaxial Flow Surfaces and Stress-Based Forming Limit Curves}

An X-ray stress measuring system was recently installed on the sheet metal formability station. This unique instrumentation allows for the direct, in situ 


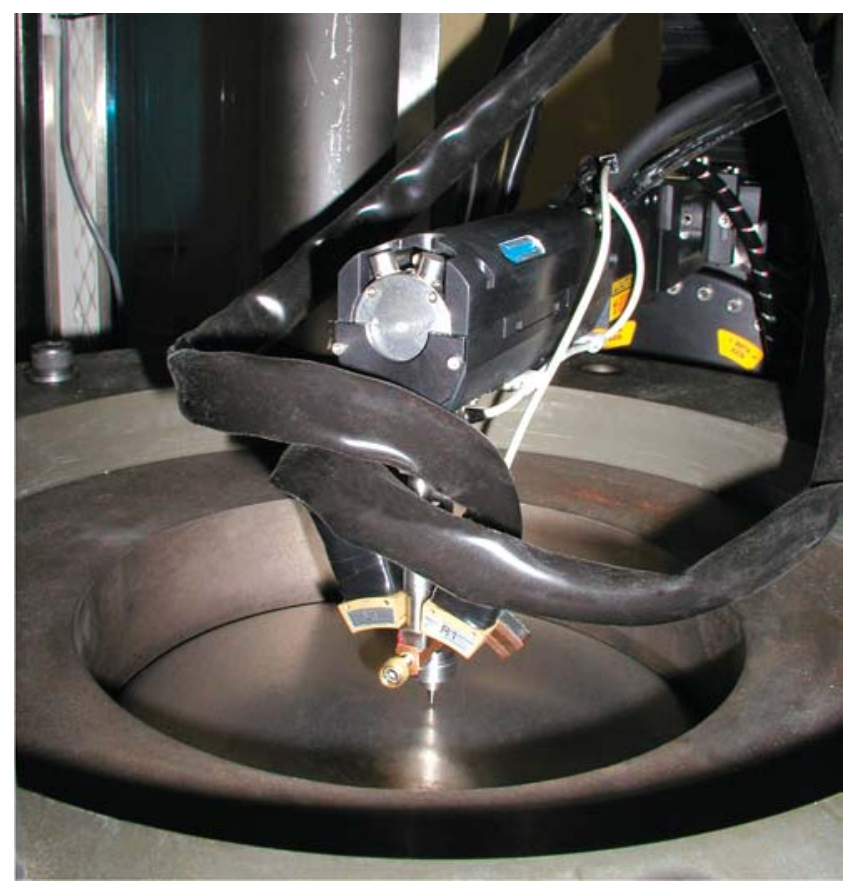

Figure 2: Newly installed X-ray stress measuring system as it takes data on sheet metal being deformed in biaxial tension.

measurement of the stress in a given direction while the sample is under multiaxial load. This year, the multiaxial stress-strain surfaces have been mapped for three aluminum sheet alloys of particular interest to the automotive industry: AA5182, AA6111 and AA6022. With this experimental system, shown in Figure 2, we have made the first-ever recorded measurements of the biaxial hardening exponent. These data will provide a substantial advantage to industry, allowing the replacement of current practices that estimate biaxial flow behavior from a series of extrapolated uniaxial tensile tests. This new approach is expected to enable more accurate designs and greater cost savings.

Press releases and technical articles have brought these new capabilities to the attention of industry. Visits and inquiries from companies such as ISG-International Steel Group, Volvo, and Avesta-Polarit have stimulated the exploration of mutually beneficial research that can produce enabling data and new insights into formability.

Accumulated plastic strain has been used for decades to describe the deformation limits of sheet metal. However, this formalism does not account for path dependence (i.e., how deformation response depends on how it is deformed). Stress, on the other hand, is an intrinsic property of metals, and the ability to describe a process in terms of critical stresses rather than strains would remove the dependence on path. Recent work at GM suggests that this approach would greatly improve finite element predictions by better defining the safe working envelope of the materials. Still needed are direct measurements of multiaxial flow surfaces, measurements that only NIST can provide.
A collaboration with GM has been developed using these new data to remove the pure isotropic and kinematic hardening models currently in use. Improvements to the modeling and limit formalisms will be directly transferred to the auto industry.

\section{NUMISHEET 2005}

A triennial international conference focusing on simulation techniques for sheet metal forming die design will be held in Detroit in 2005. An integral part of this conference is a round-robin simulation exercise where various modeling groups try to predict the final shape of a prototypical forming operation. NIST has been asked to take the key role of measuring material properties and then forming the prototype part. This year, the new NIST capability to measure stresses in situ has allowed an increase in the complexity and sophistication of the round-robin simulation. Prediction of both through-thickness residual stresses in a sheet that has undergone multiple forming operations and the stresses in orthogonal directions in a sheet under load in a die have become vital components of the benchmark for the conference, representing a "next-level" challenge for modelers to predict.

\section{Springback Prediction}

"Springback," during and after forming operations, limits the accuracy and consistency of the final part. The cup test for measuring springback is a fairly recent development and has not been completely characterized. NIST development and testing of the springback cup test has now been largely completed. A collaboration with U.S. Steel has shown that computer models that have been recently modified to account for springback can now accurately predict the final geometry of the sample. More significantly, the models come close to predicting the residual stress profiles that NIST has measured with neutron and X-ray diffraction.

Working with ASTM and industry, NIST has submitted the flat-bottomed springback cup test for consideration as a standard measurement protocol for the capacity for elastic springback. A committee, led by Ford and including both material suppliers and end-users, will consider this new test. Data generated by NIST on the robustness of the test and the sensitivity to experimental variations will form the basis of round-robin analyses.

\section{For More Information on this Topic}

L.E. Levine (Metallurgy Division, NIST); T. Gnäupel-Herold, H. Prask (NIST Center for Neutron Research); M. Shi (USS); E. Chu (ALCOA); C. Xia (Ford); T. Stoughton, M. Wenner, C.T. Wang (GM); A. Lin (Univ. of Illinois) 


\section{Breakthroughs in Magnetic Refrigeration}

In the past 10 to 15 years, there has been a substantial effort to find alternative cooling technologies to vapor compression systems. This has been driven largely by the need to reduce the high energy costs of present systems along with their use of harmful CFC refrigerants. Magnetic refrigeration technology is one such alternative. In principal, because it is a reversible process, magnetic cooling should be more efficient than the competing irreversible vapor compression systems. However, magnetic cooling technology has historically been relegated to cryogenic, high-magnetic field applications since efficiency was so poor at higher temperatures. In 2004, we made progress in identifying materials with greatly improved performance close to room temperature. For U.S. industry to take advantage of these new materials, their behavior needs to be understood and methods of proper measurement determined. We are providing the metrology to do that.

\section{Robert Shull, Virgil Provenzano, and Alexander Shapiro}

Tn 1997 a large ("giant") magnetocaloric effect was reported in the $\mathrm{Gd}_{5} \mathrm{Ge}_{2} \mathrm{Si}_{2}$ compound between $270 \mathrm{~K}$ and $300 \mathrm{~K}$, thereby generating a great deal of interest as a potential near-room temperature magnetic refrigerant. Unfortunately, large hysteretic losses occur in the same temperature range where this compound exhibits its large magnetocaloric effect, making the magnetic refrigeration with the material inefficient.

In Nature [Vol. 429, p. 853 (2004)], we reported on the discovery of an effective and straightforward method for greatly reducing (by more than 90 percent) the large hysteresis losses in $\mathrm{Gd}_{5} \mathrm{Ge}_{2} \mathrm{Si}_{2}$. This is accomplished by alloying the compound with a small amount of iron, which has the additional benefit of shifting the magnetic entropy change $\left(\Delta \mathrm{S}_{\mathrm{m}}\right)$ peak to higher temperature, from $275 \mathrm{~K}$ to $305 \mathrm{~K}$, and broadening its width. Although the addition of Fe yields comparable refrigerant capacity $(\mathrm{RC})$ values, a greater resultant value is obtained for the Fe-containing alloy when the hysteresis losses are subtracted. The Fe-containing alloy is clearly better for near-room temperature magnetic refrigeration applications.

Figure 1 shows the magnetization (M) vs. applied magnetic field $(\mathrm{H})$ data measured in the important temperature range of peak magnetocaloric effects

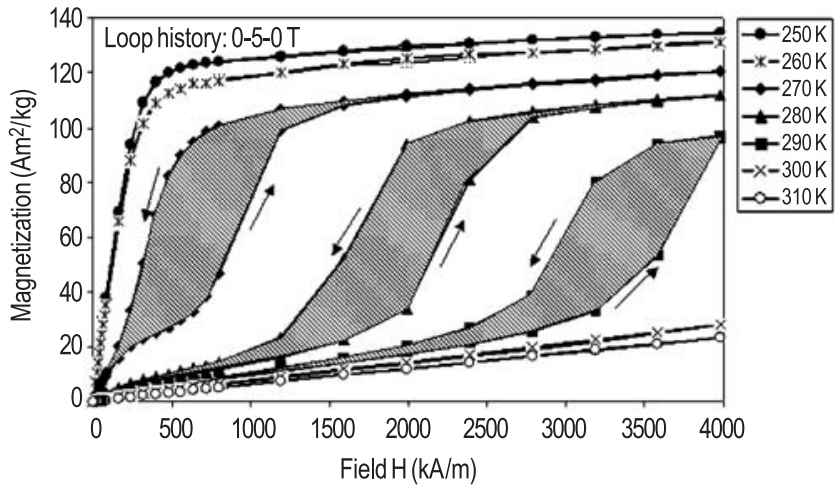

Figure 1: Magnetic hysteresis measurements showing a field-induced ferromagnetic transition and large hysteresis in the "giant magnetocaloric effect" $\mathrm{Gd}_{5} \mathrm{Ge}_{2} \mathrm{Si}_{2}$ compound.

for the $\mathrm{Gd}_{5} \mathrm{Ge}_{2} \mathrm{Si}_{2}$ compound. In this material, the enhanced values of the magnetocaloric effect have been ascribed to the existence of a field-induced crystallographic phase change in the material from a low-field paramagnetic structure to a high-field ferromagnetic crystal structure. As a consequence of this field-induced phase change, there is hysteresis in its transformation, shown by the shaded areas enclosed within the field cycles, corresponding to the cost in energy to make one cycle. The magnitude must be assessed when determining whether a particular refrigerant is useful in a given application.

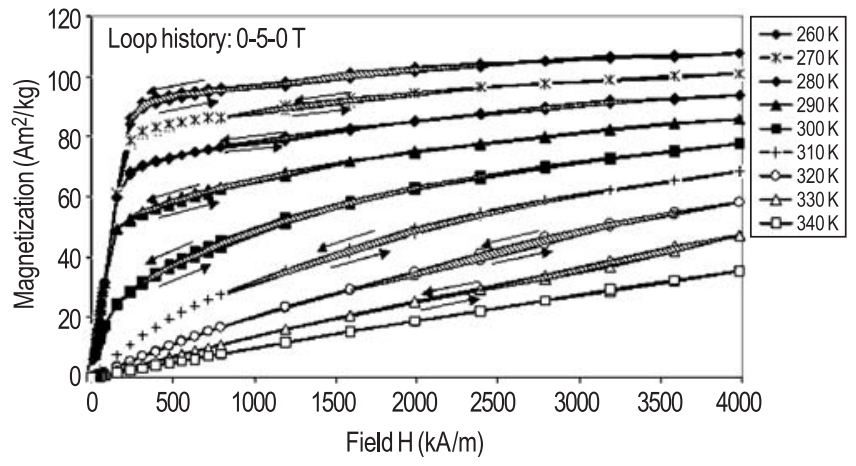

Figure 2: Magnetic hysteresis measurements showing no field-induced ferromagnetic transition and nearly zero hysteresis in the $\mathrm{Gd}_{5} \mathrm{Ge}_{1.9} \mathrm{Si}_{2} \mathrm{Fe}_{0.1}$ alloy.

We have found that by adding a small amount of Fe to the $\mathrm{Gd}_{5} \mathrm{Ge}_{2} \mathrm{Si}_{2}$ compound, the hysteresis may be significantly reduced. This effect is shown in the $\mathrm{M} v s . \mathrm{H}$ data for this alloy in the important temperature range of potential use in Figure 2, where the shaded hysteresis regions are nearly zero. 


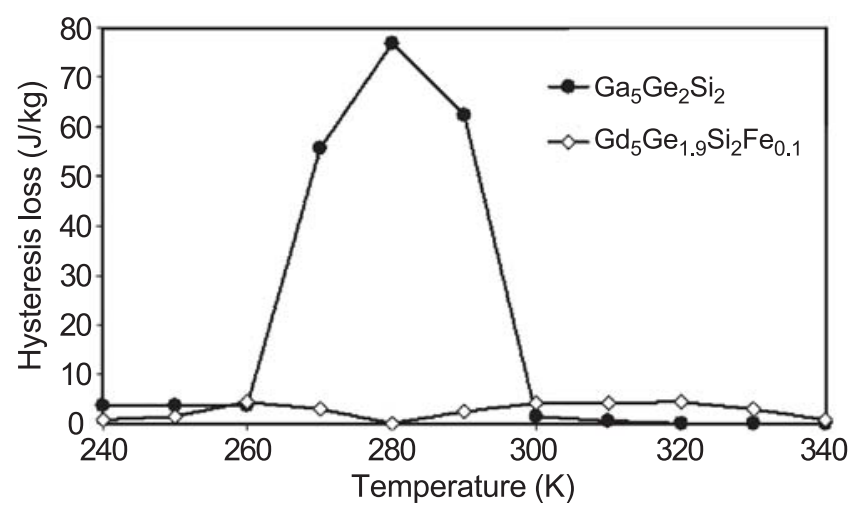

Figure 3: Hysteresis energy loss as a function of temperature for the $\mathrm{Gd}_{5} \mathrm{Ge}_{2} \mathrm{Si}_{2}$ and $\mathrm{Gd}_{5} \mathrm{Ge}_{1.9} \mathrm{Si}_{2} \mathrm{Fe}_{0.1}$ alloys.

A magnetic field is alternately applied and removed from a refrigerant during a single cooling cycle in a magnetic refrigerator, thus any magnetic hysteresis will reduce the usefulness of a material. Figure 3 shows exactly how much of a difference in hysteresis there is between the $\mathrm{Gd}_{5} \mathrm{Ge}_{2} \mathrm{Si}_{2}$ and $\mathrm{Gd}_{5} \mathrm{Ge}_{1.9} \mathrm{Si}_{2} \mathrm{Fe}_{0.1}$ alloys.

The reduced hysteresis in the quaternary alloy is due to a lack of field-induced paramagnetic-to-ferromagnetic crystallographic phase change. This change in behavior is caused by the $\mathrm{Fe}$ combining with primarily the $\mathrm{Si}$ in the ternary compound to form an Fe-rich secondary phase, thereby reducing the Si content in the majority Gd-Ge-Si phase. As a consequence, the thermodynamics of the Gd-Ge-Si alloy were changed from that of the precursor $\mathrm{Gd}_{5} \mathrm{Ge}_{2} \mathrm{Si}_{2}$ compound.

The magnetic entropy change $\left(\Delta \mathrm{S}_{\mathrm{m}}\right)$ for a $5 \mathrm{~T}$ applied field as a function of temperature for both alloys is shown in Figure 4. The computed refrigerant capacities (RC) are indicated on the figures and shown by the shaded areas. Compared to $\mathrm{Gd}_{5} \mathrm{Ge}_{2} \mathrm{Si}_{2}$, the iron-containing compound exhibits a smaller, but broader $\Delta \mathrm{S}_{\mathrm{m}}$ peak, and a $\Delta \mathrm{S}_{\mathrm{m}}$ peak at a higher temperature. Purely on this basis, $\mathrm{Gd}_{5} \mathrm{Ge}_{2} \mathrm{Si}_{2}$ is the superior material, but the Fe-containing alloy might be attractive for higher temperature applications due to its higher temperature peak.

One method to account for the hysteresis losses of each alloy is to simply subtract it from the corresponding refrigerant capacity value. Subtraction of the average hysteresis in the temperature range of the $\Delta \mathrm{S}_{\mathrm{m}}$ peak for each alloy $(\sim 65 \mathrm{~J} / \mathrm{kg}$ and $\sim 4 \mathrm{~J} / \mathrm{kg}$, respectively, for the ternary and quaternary alloys) from the corresponding refrigerant capacity values yielded approximate values of $240 \mathrm{~J} / \mathrm{kg}$ for the Fe-free alloy and $355 \mathrm{~J} / \mathrm{kg}$ for the Fe-containing alloy. Consequently, when the hysteresis losses are taken into account, it is clear that the $\mathrm{Gd}_{5} \mathrm{Ge}_{1.9} \mathrm{Si}_{2} \mathrm{Fe}_{0.1}$ alloy is a much
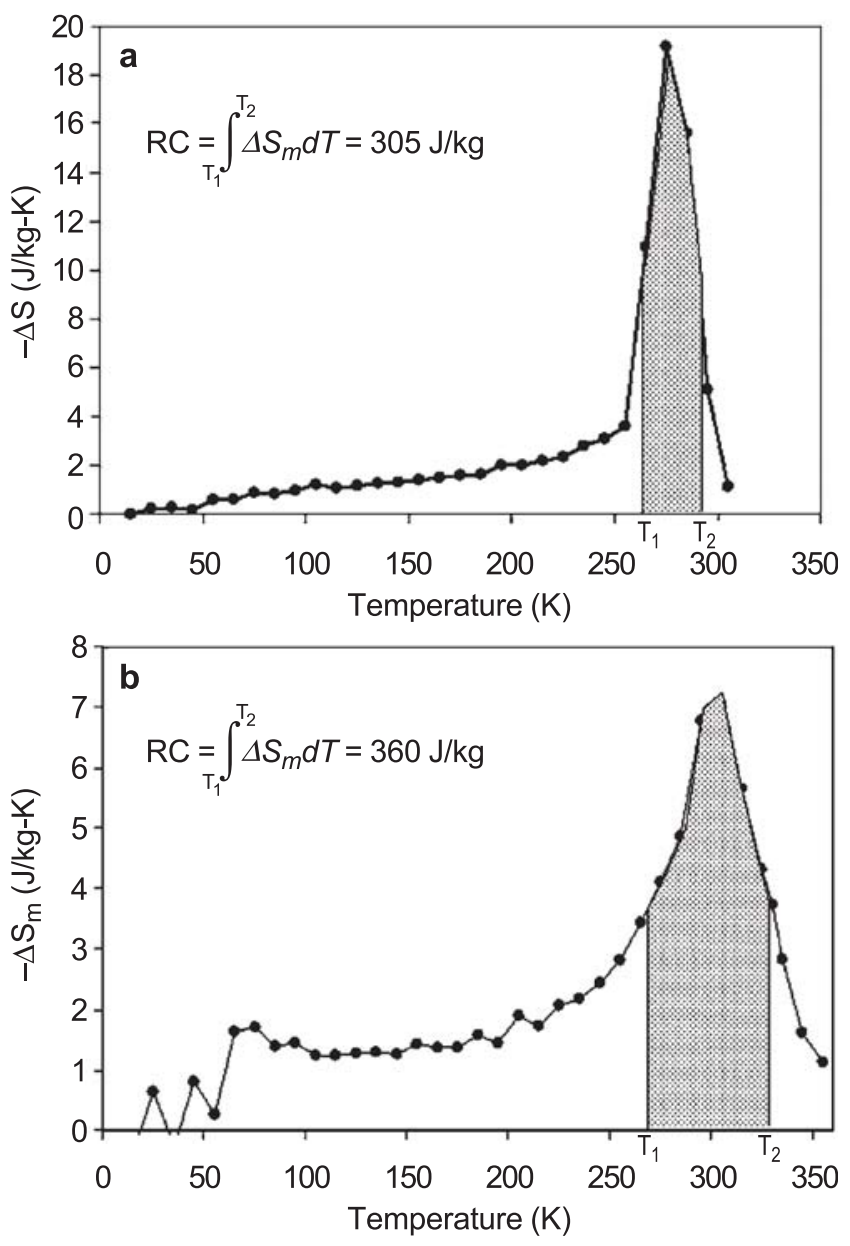

Figure 4: Computed $\Delta S_{m}[$ for $\Delta H=3980 \mathrm{kA} / \mathrm{m}(5 \mathrm{~T})]$, normalized with respect to sample mass, of the $\mathrm{Gd}_{5} \mathrm{Ge}_{2} \mathrm{Si}_{2}$ compound (top) and of the $\mathrm{Gd}_{5} \mathrm{Ge}_{1.9} \mathrm{Si}_{2} \mathrm{Fe}_{0.1}$ alloy (bottom), plotted as a function of temperature showing the presence of peaks centered near $270 \mathrm{~K}$ and $305 \mathrm{~K}$ respectively for $\mathrm{Gd}_{5} \mathrm{Ge}_{2} \mathrm{Si}_{2}$ and $\mathrm{Gd}_{5} \mathrm{Ge}_{1.9} \mathrm{Si}_{2} \mathrm{Fe}_{0.1}$.

better magnetic refrigerant than the $\mathrm{Gd}_{5} \mathrm{Ge}_{2} \mathrm{Si}_{2}$ compound despite its lower $\Delta \mathrm{S}_{\mathrm{m}}$ peak value. Most prior evaluations of magnetic refrigerants have disregarded the material hysteresis losses, and in some materials these losses can be quite large; this example shows the weakness in that approach.

"The results are pretty amazing," states Michael DiPirro [Science News 165, 405 (2004)], a cryogenic engineer at the NASA Goddard Space Flight Center in Greenbelt, Maryland.

\section{For More Information on this Topic}

Virgil Provenzano, Robert D. Shull, and Alexander J. Shapiro, "Reduction of hysteresis losses in the magnetic refrigerant $\mathrm{Gd}_{5} \mathrm{Ge}_{2} \mathrm{Si}_{2}$ by the addition of Iron," Nature 429, 853 (2004). 


\section{Grand Challenges in Nanomagnetics: High Coercivity FePt Alloys for Future Perpendicular Magnetic Data Storage}

The magnetic data storage industry is seeking to sustain the 30-year trend of exponentially increasing storage density. This must be achieved at the same time as maintaining the stability of the recorded data. The Metallurgy Division at NIST is collaborating with Seagate Technology to develop the processes required to produce patterned media of high-magnetic coercivity that will meet this challenge.

Jonathan J. Mallett, Thomas P. Moffat, and William F. Egelhoff, Jr.

$\mathrm{C}$ onventional magnetic data storage media are based on granular films in which many fine grains are used to define each magnetic bit. The minimum number of grains per bit is limited by the irregularity of the shape and spacing of the grains, which produces irregular boundaries between bits. This irregularity results in noise in the read signal and can only be constrained at an acceptable level by maintaining a fixed minimum number of grains per bit. The natural approach to increasing data storage density has been to reduce the grain size, while maintaining the same number of grains per bit. The limit to this approach occurs when the magnetic energy barrier to the reversal of magnetization of each grain approaches the energy of random thermal fluctuations. At this point, the medium is no longer stable against spontaneous magnetization reversal and consequential data loss.

The grain size at which this limit occurs depends on the magnetocrystalline anisotropy energy of the

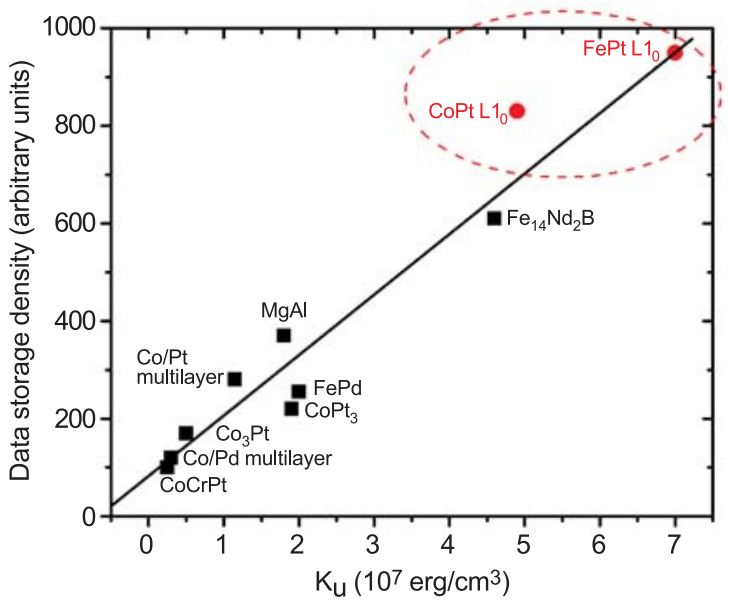

Figure 1: The dependence of storage density on magnetocrystalline anisotropy energy. The most promising materials are FePt and CoPt. magnetic material. FePt in its $\mathrm{L}_{0}$ phase has a sufficiently large anisotropy energy to allow magnetically stable grains of $5 \mathrm{~nm}$ diameter (Figure 1). Furthermore, FePt deposited by a variety of means typically forms grains approaching this size. Unfortunately, the annealing treatment required to transform the as-deposited A1-structured FePt to the desirable $\mathrm{L} 1_{0}$ phase is widely found to result in an increase in grain size to $100 \mathrm{~nm}$. Current efforts at NIST focus on developing a method of electrochemically depositing FePt into a regular patterned template, circumventing the problem of grain growth. Simultaneously, the imposed regularity of the magnetic cells opens the possibility of addressing single cells (i.e., of using one "grain" per bit). The estimated achievable density from such an approach is 7 Tbits per square inch, which is 100 times higher than current storage densities. Electrodeposition is an obvious choice for deposition into high aspect ratio templates, since it does not suffer from the shadowing effect that characterizes vacuum deposition techniques.

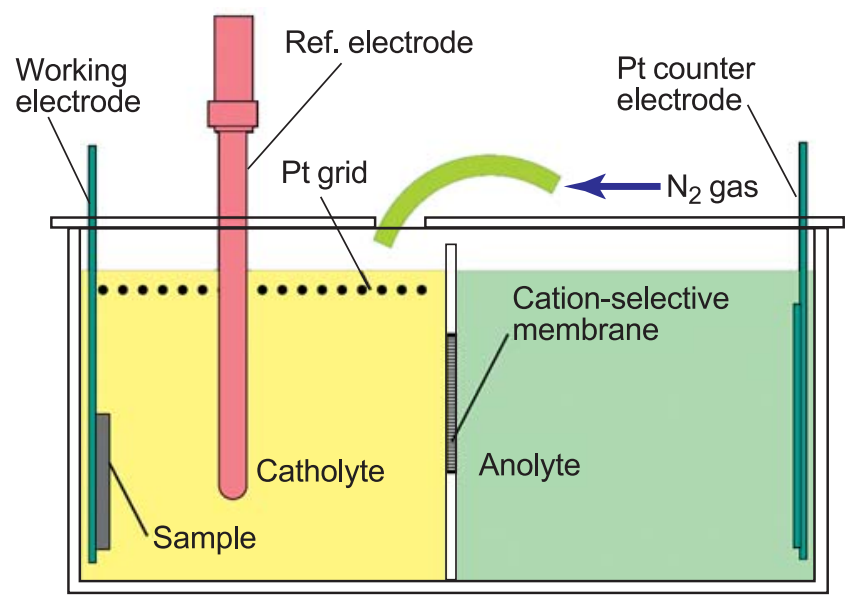

Figure 2: The double cell, designed to minimize the concentration of $\mathrm{Fe}^{3+}$ and dissolved $\mathrm{O}_{2}$ in the FePt plating solution.

Electrodeposition of FePt from aqueous solutions presents many challenges. The double cell shown in Figure 2 has been developed to address the problem of the instability of $\mathrm{Fe}^{2+}$ solutions, which readily oxidize to produce insoluble $\mathrm{Fe}(\mathrm{OH})_{3}$. The plating solution, containing $\mathrm{FeCl}_{2}$ and $\mathrm{PtCl}_{4}$ (left) is separated from the anode by a cation selective membrane, preventing the oxidation of $\mathrm{Fe}^{2+}$ to $\mathrm{Fe}^{3+}$ that would otherwise occur at the anode. $\mathrm{Fe}^{3+}$ formation by oxidation with air is also minimized by blanketing the cell in nitrogen, and residual $\mathrm{Fe}^{3+}$ is reduced to $\mathrm{Fe}^{2+}$ by an auxiliary platinum grid electrode. 


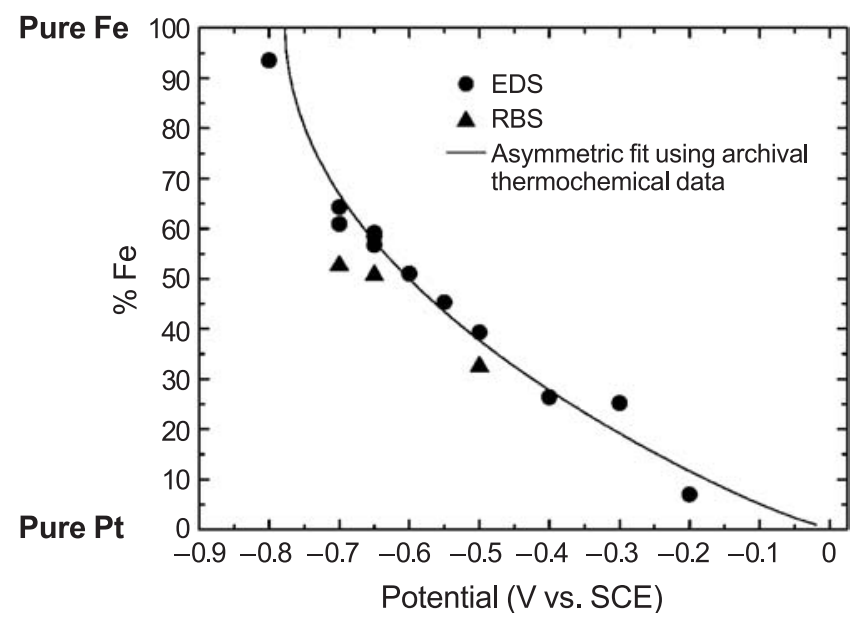

Figure 3: The theoretical and experimental dependence of composition on the applied potential.

The alloy composition of $300 \mathrm{~nm}$ thick films deposited from this solution was found to depend mainly on the applied potential and to be insensitive to the concentration of the solution components. A thermodynamic regular solution model was used to describe the dependence of film composition on applied potential. The comparison of theory to experimental data can be seen in Figure 3.

The deposited films were found to be remarkably smooth, with RMS roughness values less than $5 \mathrm{~nm}$ for micron-thick deposits. This was surprising given the large platinum overpotential (supersaturation), which usually results in growth instabilities and consequent roughening.

The control of crystal orientation is an essential consideration for high-density recording applications. A transition in the recording industry is currently in progress to perpendicular media, in which the bits are magnetized perpendicular to the plane of the medium. Maximum advantage can be derived from $\mathrm{L} 1_{0} \mathrm{FePt}$ as a perpendicular medium when it is correctly aligned with its magnetic easy-axis perpendicular to the substrate plane. A careful choice of substrate and annealing parameters is required to recrystallize the as-deposited random fcc alloy to appropriately oriented $\mathrm{L}_{0}$.
(001)

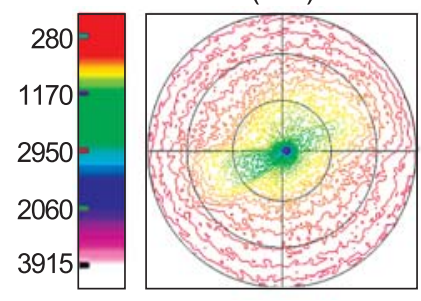

(110)
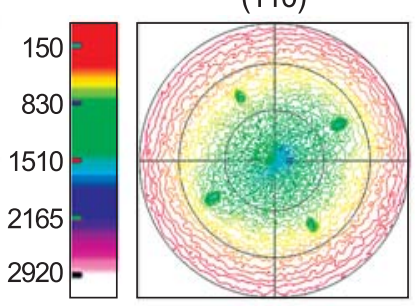

Figure 4: X-ray pole figures showing favorably oriented FePt $L 1_{0}$.
FePt electrodeposited onto a $\mathrm{Cu}(001)$ substrate has recently shown great promise. X-ray diffraction revealed the transition to $\mathrm{L}_{0}$ upon annealing, and $\mathrm{X}$-ray pole figures indicated favorable orientations. Figure 4 shows FePt $\mathrm{L}_{0}$ (001) and (110) pole figures. The (001) figure indicates perpendicular orientation of the magnetic easy-axis (the c-axis), while the (110) figure indicates an in-plane texture. The in-plane texture is an added advantage, as it results in a narrower switching field distribution.

Copper additions to the alloy have been found to lower the $\mathrm{A} 1 / \mathrm{L}_{0}$ phase transition temperature by up to $90{ }^{\circ} \mathrm{C}$. It may be speculated that interdiffusion of copper from the substrate during the anneal allowed the recrystallization to proceed from the interface with the substrate. It is likely that this would allow the film to replicate the orientation of the substrate.

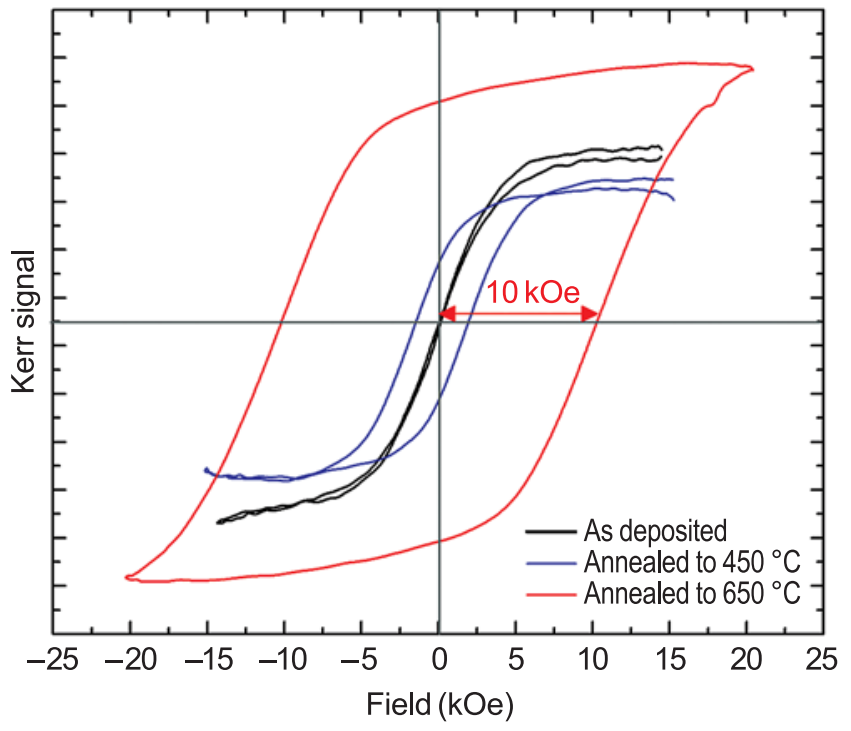

Figure 5: Magnetic hysteresis measurements showing a $10 \mathrm{kOe}$ coercivity.

Magnetic hysteresis measurements performed using a Kerr magnetometer are shown in Figure 5. The magnetic coercivity of $10 \mathrm{kOe}$ is comparable to figures quoted in the literature for vacuum-deposited FePt. It is believed to be the highest value reported for an electrodeposited film.

Current efforts focus on controlling the interdiffusion between substrate and film to minimize loss of magnetization in the alloy, and on moving from planar films to through-mask deposited arrays of FePt pillars.

\section{For More Information on this Topic}

W.F. Egelhoff, Jr. (Metallurgy Division, NIST); E.B. Svedberg (Seagate Research, Pittsburgh) 


\section{On-Chip Interconnects: Extending Performance of Sub-100 nm Lines}

Conductors in on-chip metallizations are now reaching dimensions so small that defective seed layers are impacting manufacturing yields, and electron scattering on surfaces and grain boundaries is reducing electrical transport in the buried wires. Our goal is to provide tools to overcome these barriers. Recent efforts have quantified sources of the increased resistivity in wires made of silver, the most conductive of all metals, demonstrated seedless processing routes for copper wires, and improved understanding and modeling of the superfill fabrication process.

\section{Daniel Josell and Thomas P. Moffat}

$\mathrm{T}_{\mathrm{i}}^{\mathrm{h}}$ he steady reduction of transistor dimensions in integrated circuits has been accompanied by similar size reductions of the on-chip interconnects that carry electrical signals, pushing the industrial technology for copper seed deposition close to its limit for defect-free sidewall coverage. Defects in seed layers, which arise from limitations in existing sputter technology, lead to voiding during electrodeposition of the copper metallization.

Additionally, with the dimensions of the copper wires in these metallizations now approaching the intrinsic mean-free-path length of the conduction electrons, scattering on the wire surfaces has begun to significantly reduce electron transport and, thus, the associated electrical conductance of the wires. With grains in these conductors similarly sized, a reduction is also to be expected from grain boundary scattering. While the penalty for both effects is increased power dissipation and reduced clock speed, the appropriate approach for mitigation requires quantitative determination of the relative sizes of the contributions.
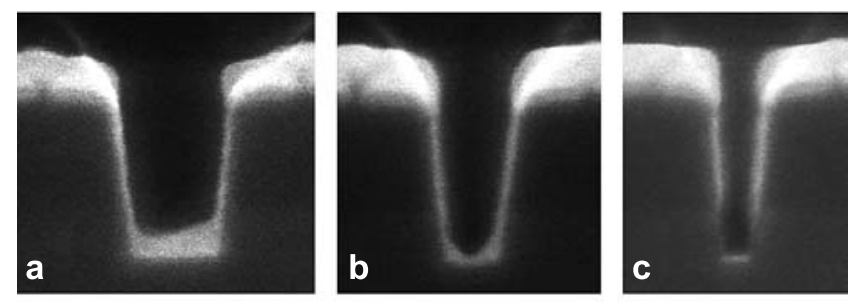

$100 \mathrm{~nm}$

Figure 1: $\mathrm{Ti} / \mathrm{Pd} / \mathrm{Ag}$ seed layer in sub-100 $\mathrm{nm}$ deep trenches.

\section{Technical Details}

A tri-layer titanium, palladium and silver seed (Figure 1) was shown to yield smooth, conductive surfaces for the electrodeposition of silver wires for the electrical properties study. The poor seed coverage that is visible toward the bottom of the smallest trench (Figure 1c) is a technical challenge noted in the International Technology Roadmap for Semiconductors. Such seed defects motivated the "seedless" ruthenium barrier-based process detailed in last year's report and continued by this year's demonstration of seedless copper superfill in trenches with ruthenium or iridium barriers (Figure 2) deposited by perfectly conformal atomic layer deposition (ALD).

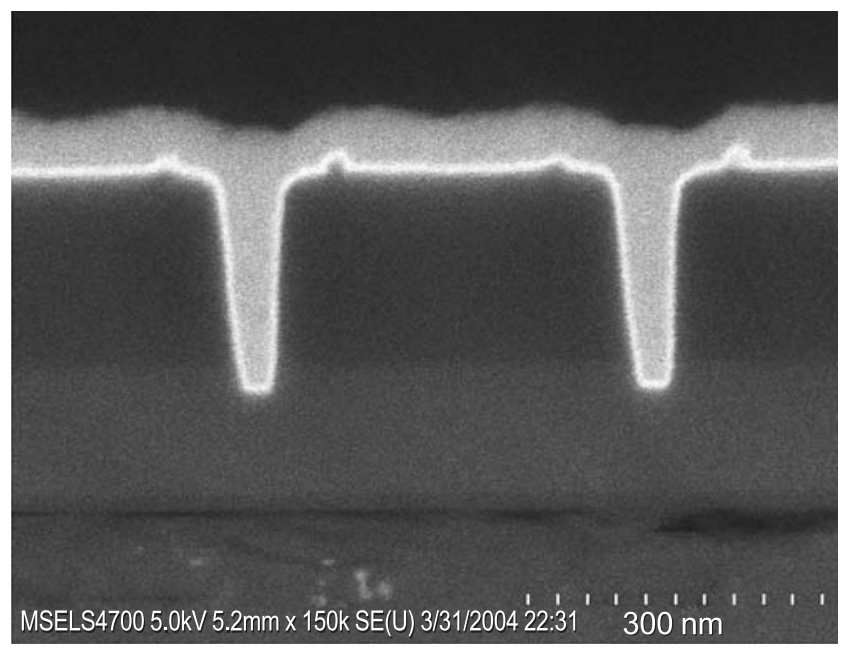

Figure 2: Trenches containing copper that was electrodeposited directly on an ALD iridium barrier (thin bright layer).

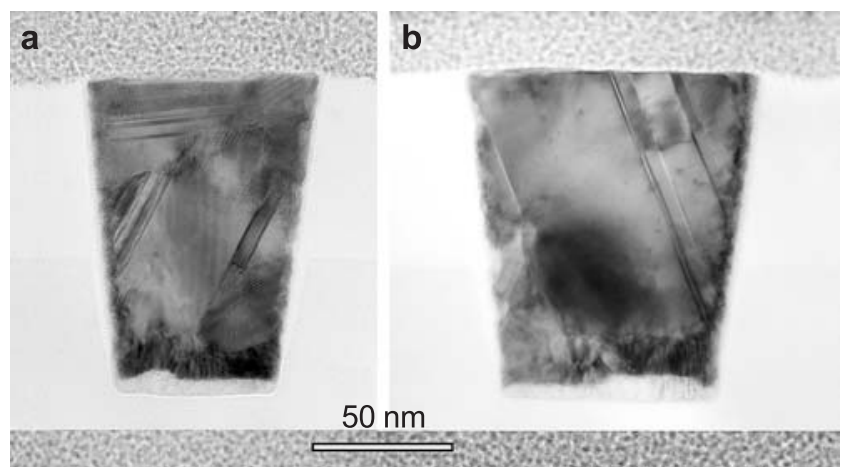

Figure 3: Cross-section views of silver wires fabricated by electrodeposition in the seeded trenches followed by removal of the metal from the field (transmission electron microscope). 
For the electrical studies, silver wires (Figure 3) were fabricated by silver electrodeposition on the tri-layer seeds (Figure 1), using a superfill process developed in the Metallurgy Division, followed by removal of the metal in the field adjacent to the wires through chemicalmechanical planarization and ion polishing.

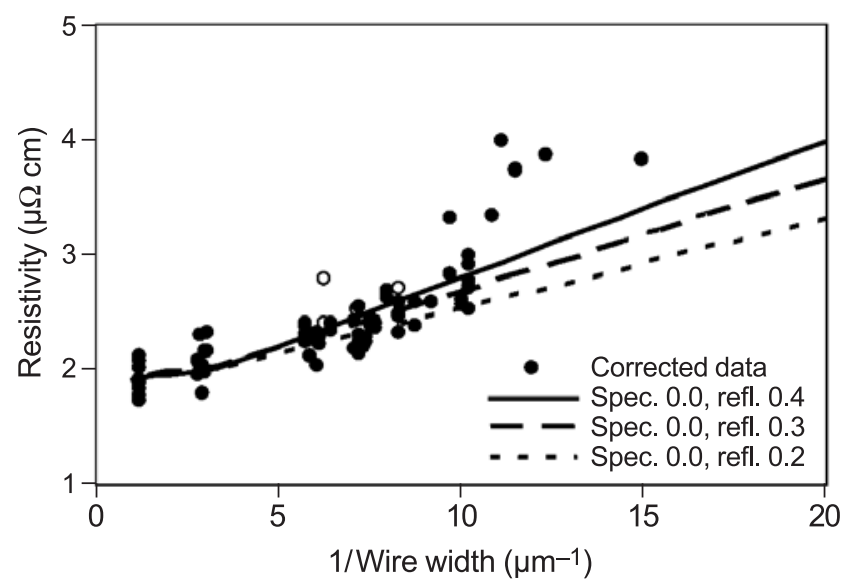

Figure 4: Electrical resistivities of $300 \mathrm{~nm}$ tall silver wires compared to predictions that account for surface scattering with varying amounts of grain boundary scattering. Resistivities for wires less than $100 \mathrm{~nm}$ wide are impacted by defects.

The wires were studied in a standard four-point probe geometry that permitted measurement of wire resistances. Resistivities, obtained from the resistances using measured wire dimensions, increased significantly with decreasing wire width (Figure 4). To assess the origin of the resistivity increase, the Fuchs-Sondheimer analysis for diffuse scattering of electrons on surfaces was extended to permit analysis in the presence of both specular and diffuse scattering. The resulting equations, along with a previously published equation for grain boundary scattering, permitted quantitative evaluation of the experimental data. Significantly, modeling of the data showed that the increase of resistivity with decrease of wire size arises as much from scattering on grain boundaries as from scattering on the wire surfaces. This indicates that efforts to mitigate size effects must increase grain size as well as surface specularity if they expect to be more than modestly successful.

The continuing industrial need for predictive simulation of feature filling spurred experiment and modeling of the superfill process itself. Consumption of adsorbed accelerator, a detrimental deviation from the surface segregation behavior that is responsible for the superfill process, was measured. Inclusion of such consumption in our Curvature Enhanced Accelerator Coverage (CEAC) model and computer code have made the filling predictions even more accurate.

This research has continued to impact industry, indicated by requests for our superfill code from Intel,

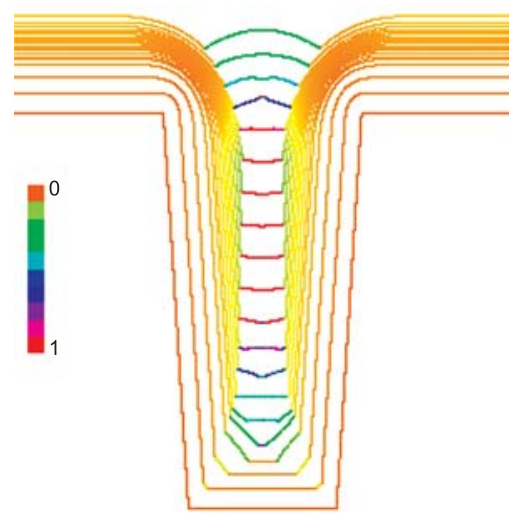

Figure 5: Simulations of superconformal feature filling now account for consumption (incorporation) of adsorbed catalyst.

Applied Materials, ST Microelectronics, and ATMI; an invited article on superfill in the IBM Journal of Research and Development; and invited presentations given at Cookson-Enthone, in addition to publications and presentations at conferences.

\section{Selected Project Publications for FY2004}

T.P. Moffat, D. Wheeler, M. Edelstein and D. Josell, "Superconformal Film Growth: Mechanism and Quantification," IBM J. Res. and Dev., in press.

D. Josell, C. Burkhard, Y. Li, Y.-W. Cheng, R.R. Keller, C.A. Witt, D. Kelley, J.E. Bonevich, B.C. Baker, T.P. Moffat, "Electrical Properties of Superfilled Sub-Micrometer Silver Metallizations," J. Appl. Phys. 96 (1), 759-768, (2004).

D. Wheeler, T.P. Moffat, G.B. McFadden, S. Coriell and D. Josell, "Influence of Catalytic Surfactant on Roughness Evolution During Film Growth," J. Electrochem. Soc. 151 (8), C538-C544, (2004).

T.P. Moffat, D. Wheeler, and D. Josell,

"Electrodeposition of Copper in the SPS-PEG-Cl Additive System: I. Kinetic Measurements: Influence of SPS," J. Electrochem. Soc. 151 (4), C262-C271, (2004).

W.J. Evans, D.G. Giarikos, D. Josell, and J.W. Ziller, "Synthesis and Structure of Polymeric Networks of Silver Hexafluoroacetylacetonate Complexes of THF, Toluene, and Vinyltrimethylsilane," Inorg. Chem. 42, 8255-8261, (2003).

\section{For More Information on this Topic}

D. Josell, T.P. Moffat (Metallurgy Division, NIST); G. McFadden (Mathematical and Computational Sciences Division, NIST); R.R. Keller, Y.-W. Cheng (Materials Reliability Division, NIST); C. Witt (Cookson-Enthone); C. Burkhard, Y. Li (Clarkson University); D. Wheeler (University of Maryland); T.K. Aaltonen, M. Ritala, M. Leskelä (University of Helsinki, Finland) 


\section{The Structural Steel of the World Trade Center Towers}

\begin{abstract}
In August of 2002, NIST took responsibility for the investigation of the World Trade Center disaster. The investigation objectives include determining: why the buildings collapsed; the procedures and practices used in the design, construction, operation and maintenance of the buildings; and areas in codes and practices that warrant revision. The eight interdependent projects include Project 3 - Analysis of Structural Steel, led by the Materials Science and Engineering Laboratory. The objective of the project is to determine and analyze the properties and quality of the steel, weldments, and connections from steel recovered from the World Trade Center.
\end{abstract}

Stephen W. Banovic, Richard J. Fields, Timothy J. Foecke, William E. Luecke, J. David McColskey, Christopher N. McCowan, Thomas A. Siewert, and Frank W. Gayle

\footnotetext{
Tn terms of the steel used, the World Trade Center was very complex. Plans called for steels of fourteen different yield strengths between $36 \mathrm{ksi}$ and $100 \mathrm{ksi}$. Many of these steels were proprietary and were not supplied to any existing ASTM specification. To further complicate matters, four different fabricators provided steel for the upper stories, and each used steel from more than one supplier. Most of the records that documented the steel actually used were either not preserved or destroyed in the collapse.
}

The MSEL Metallurgy and Materials Reliability Divisions have determined properties of the steel recovered from the WTC site and characterized failure modes associated with pre-collapse damage. This information has been provided for use in models of the building response to airplane impact and fire. In 2004, all major tasks were completed.

\section{Task 1 - Collect and Catalog Physical Evidence}

The Structural Engineers Association of New York (SEAoNY), later supplemented by NIST personnel, spent countless hours selecting steel in the New Jersey recycling yards for later forensic analysis. After shipment to NIST, the Metallurgy Division cataloged the 236 recovered steel components. Since many pieces were stamped and painted with unique serial numbers correlated with the building plans, it was often possible to identify the exact original location in the building.

As a second part of this task, we also researched contemporaneous construction documents and steel specifications. This task helped identify steel suppliers and fabricators, as well as material substitutions.

\section{Task 2 - Categorize Failure Mechanisms Based on Visual Evidence}

The damage to the steel components of the buildings represents an important check on the accuracy of the fire and impact modeling, with the caveat that the recovered steel could have been damaged before the collapse, during the collapse, or during the salvage efforts.

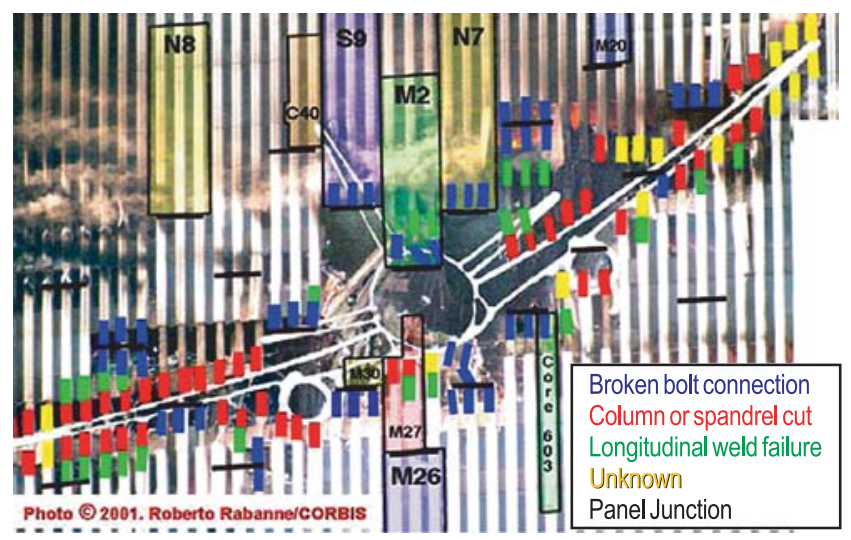

Figure 1: Enhanced image of the impact zone has allowed determination of failure modes in the perimeter columns. An outline of the plane is superimposed. Steel panels recovered for the investigation are highlighted in color.

Image enhancement techniques were used to study photographs of the impact zones of the towers to establish the failure mechanisms of the perimeter columns. By making montages of many different images, it was possible to produce a nearly smoke-free, composite view of the columns that the aircraft struck. The columns failed by several different mechanisms. One important result was the demonstration that several key impact-zone columns in the NIST inventory were damaged primarily in the aircraft impact, and not in the subsequent collapse or recovery efforts. Figure 1 is a map of the impact zone of WTC 1 that illustrates the nature of the damage to the individual columns.

\section{Tasks 3 \& 4 - Determine Steel Properties to Support Airplane Impact and Structure Performance Studies; Correlate Properties with Those Specified in the Construction}

One important part of this task was to assess the quality of steel and determine whether it met the original specifications. In support of this goal, the group conducted more than 200 room-temperature tensile tests on samples from 39 different building components, which represented 
all the steels relevant to the investigation. Tests indicate that the steels generally met or exceeded the specified minimum strengths (Figure 2).

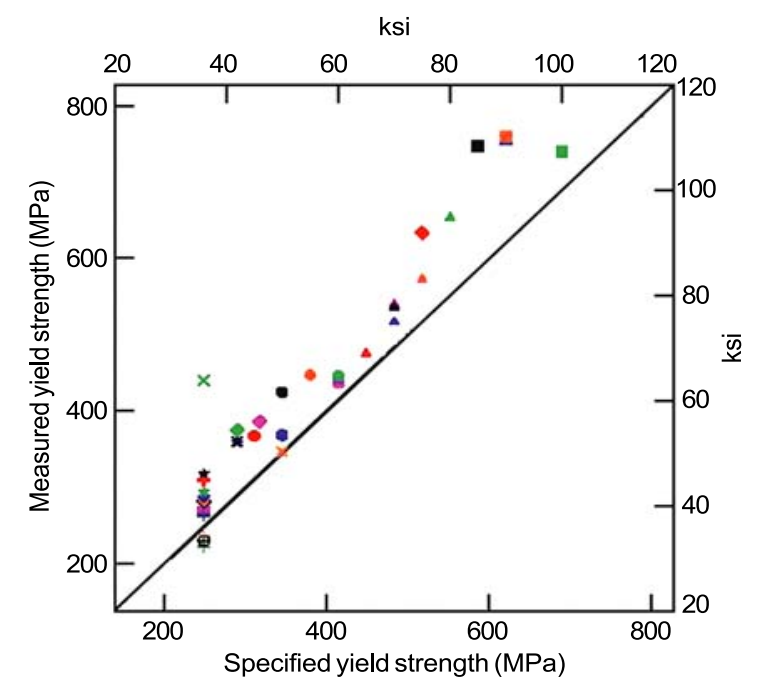

Figure 2: Yield strength - measured vs. specified minimum. The few anomalous data can be attributed to damage incurred in the collapse that removed the yield point behavior, or to the natural and accepted strength variability of structural steel.

Steel specifications also regulate the chemical compositions. More than 350 different specimens, representing nearly every relevant steel component in the building, were analyzed for chemical composition and all of the sections relevant to the investigation. The chemical analysis helped confirm archival information that an American steel mill supplied steel for parts of the perimeter columns. The analyses also support the conclusion that the steels in the buildings met the specifications called for in the building plans.

The strength of steel increases as the deformation rate increases. Accurately modeling these rate effects is essential for estimating the energy and momentum of the aircraft remnants which damaged the core columns. To establish the strain rate sensitivity of the relevant steels, we have conducted more than 100 tests on specimens from both the perimeter and core columns at rates up to $2000 \mathrm{~s}^{-1}(200,000 \%$ deformation per second).

Ultimately, the fires in the towers weakened the steel structure leading to collapse. We conducted more than 100 high-temperature tensile and creep tests to provide models of the deformation of structural steel at elevated temperatures for use in models of the building response to the fires.

\section{Task 5 - Analyze Steel to Estimate Temperature}

Just as the nature of the deformation and failure of the recovered steel reveals information about the impact and collapse, the steel can also contain evidence of its exposure to the elevated temperatures in the fire. The situation is made more complex because much of the steel was also exposed to extended fires in the rubble before recovery. Several different methods, both conventional and novel, were examined for estimating high-temperature excursions seen by the steel. Only one method proved to be robust and easy to implement: paint on steels that reached temperatures over $250^{\circ} \mathrm{C}$ cracked from the difference in thermal expansion between the paint and the steel.

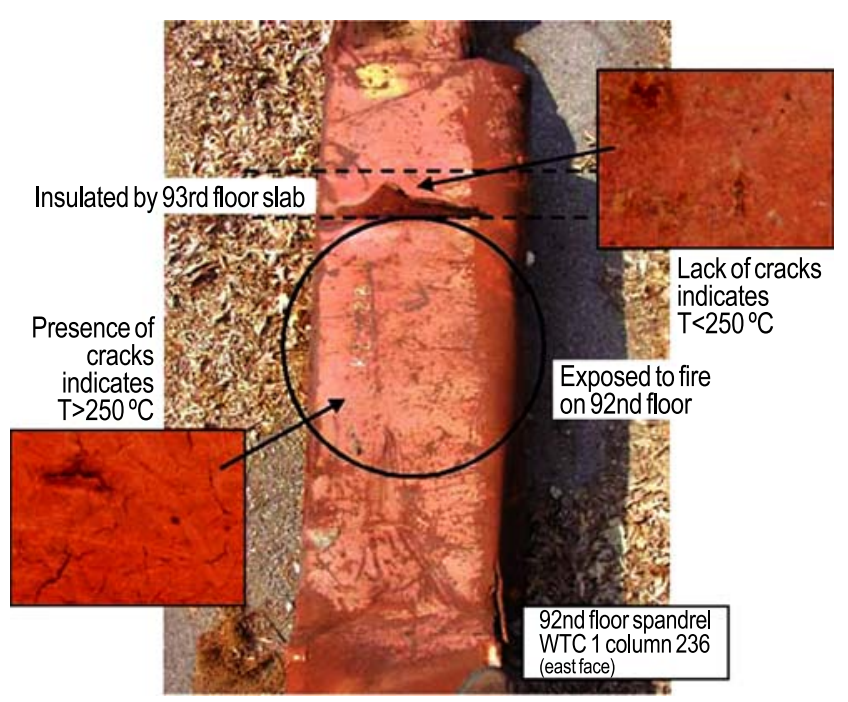

Figure 3: Illustration of the method to establish the temperature excursions of recovered steel. The segment of the column protected by the floor slab remained at lower temperature.

From photographs showing fire in windows, all recovered columns that may have been exposed to pre-collapse fire were identified and characterized using the paint crack technique. Figure 3 illustrates one of the columns that showed evidence of having exceeded $250{ }^{\circ} \mathrm{C}$.

\section{Outputs}

Outputs for the project include parts of the June 2004 Progress Report on the World Trade Center Disaster (NIST SP 1000-5) and numerous memos for contractors that explain how to employ Project 3 data and deformation models. The final NIST investigation report will be released in the spring of 2005 .

\section{For More Information on this Topic}

T.A. Siewert (Materials Reliability Division, NIST); F.W. Gayle (Metallurgy Division, NIST) 


\section{Advanced Manufacturing Processes}

The competitiveness of U.S. manufacturers depends on their ability to create new product concepts and to speed the translation from concept to market while decreasing product cost. This is equally true for well-established "commodity" industries, such as automotive and aerospace, and rapidly growing or emerging industries, such as biotechnology and nanotechnology. For existing products, manufacturing is a critical step in reducing product cycle time. Rapid, low-cost development of manufacturing processes is needed to incorporate new materials into complex product shapes with higher performance at equivalent or lower cost as the competing, established materials and methods. For innovative product concepts, new materials with increasing functionality are needed to translate these concepts to reality.

To realize such improvements in materials and manufacturing, MSEL is developing robust measurement methods, models, standards, and materials and process data needed for design, monitoring, and control of manufacturing processes. A growing challenge is being able to design, monitor, and control such materials and manufacturing processes at size scales from nanometers to meters. The Advanced Manufacturing Processes Program focuses on the following high-impact areas:

- Combinatorial, high-throughput methods for materials ranging from thin films and nanocomposites to micro- and macroscale material structures;

- Industry-targeted R\&D centered on unique measurement facilities in forming of lightweight metals for automotive applications, polymer processing, and high-speed machining;

- Innovative testbeds for emerging materials, including carbon nanotubes and fuel cells;

- National traceable standards having a major impact on trade, such as hardness standards for metals and process standards for polymers; and

- Innovative, physics-based process modeling tools.

Our research is often conducted in close collaboration with industrial consortia and standards organizations. These collaborations not only ensure the relevance of our research, but also promote rapid transfer of our research to industry for implementation. Three projects focused on Advanced Manufacturing Processes are highlighted below.

\section{NIST Combinatorial Methods Center (NCMC)}

The NCMC develops novel high-throughput measurement techniques and combinatorial experimental strategies specifically geared towards materials research.
These tools enable rapid acquisition and analysis of physical and chemical data, thereby accelerating the pace of materials discovery and knowledge generation. By providing measurement infrastructure, standards, and protocols, and expanding existing capabilities relevant to combinatorial approaches, the NCMC lowers barriers to the widespread industrial implementation of this new R\&D paradigm. MSEL uses a two-pronged strategy for accelerating the development and implementation of these approaches: an active intramural R\&D program that demonstrates the ability of combinatorial methods to produce cutting-edge scientific research and an ambitious outreach activity; key to this effort is the validation of these approaches with respect to traditional "one at a time" experimental strategies.

\section{Forming of Lightweight Metals}

Automobile manufacturing is a materials intensive industry that involves about $10 \%$ of the U.S. workforce. In spite of the use of the most advanced, cost-effective technologies, this globally competitive industry has major productivity issues related to measurement science and data. Chief among these is the difficulty of designing stamping dies for sheet metal forming. An ATP-sponsored workshop (“The Road Ahead,' June 20-22, 2000) identified production of working die sets as the main obstacle to reducing the time between accepting a new design and actual production of parts. This is also the largest single cost (besides labor) in car production. To benefit from weight savings enabled by new high-strength steels and aluminum alloys, a whole new level of formability measurement methods, models, and data is needed, together with a better understanding of the physics behind metal deformation. MSEL is working with U.S. automakers and their suppliers to fill this need.

\section{Polymer Processing}

Polymers have become ubiquitous in the modern economy because of their processability, high functionality, and low cost. However, these materials can exhibit complex and sometimes catastrophic responses to the forces imposed during manufacturing, thereby limiting processing rates and the ability to predict ultimate properties. The focus of our polymer research is on microfluidics and microscale processing, modeling of processing instabilities, and on-line process monitoring of polymers. Our unique extrusion visualization facility combines in-line microscopy and light scattering for the study of polymer blends, extrusion instabilities, and the action of additives. These measurements are carried out in close collaboration with interested industrial partners.

Contact: Carol A. Handwerker (Metallurgy Division) 


\section{Sheet Metal Forming for Automotive Applications: Anelasticity and Springback Prediction}

Increased computing power coupled with finite element modeling methods (FEM) has brought springback-compensated die design within reach of the automotive industry. In developing this technology, industry has determined that better measurement methods and an improved understanding of the influence of large plastic strains on the properties and behavior of materials during springback is required. This project seeks to develop the understanding, measurements, and data to meet this need.

\section{Richard E. Ricker}

\begin{abstract}
U.S. Council for Automotive Research (USCAR) A Consortium on Springback Prediction has developed finite element springback prediction models to reduce die design and tryout costs. However, these models consistently underpredict springback. Three alloy properties could vary with plastic strain and contribute to prediction errors: (1) elastic modulus, (2) anelastic modulus, and (3) strain-dependent anelastic behavior (reverse creep or plastic hysteresis). Currently, FEM modelers compensate for these errors with arbitrary constants estimated from trials or prior experience. The objectives of this study are to determine the origin of these errors, evaluate relevant measurement methods, and provide materials data that improves FEM springback prediction codes.
\end{abstract}

In FY 2003, we demonstrated that the large biaxial strains typical of forming can significantly reduce the elastic modulus of $\mathrm{Al}$ alloys $(18 \%)$ and that texture changes cannot explain this behavior. In FY 2004, finite element models were used to analyze the results of NIST 3-point bend springback measurements. Combining these results with modulus data obtained in FY 2003, it was determined that the strain and elastic modulus variations are too small to measure in these experiments. During FY 2004 these results were

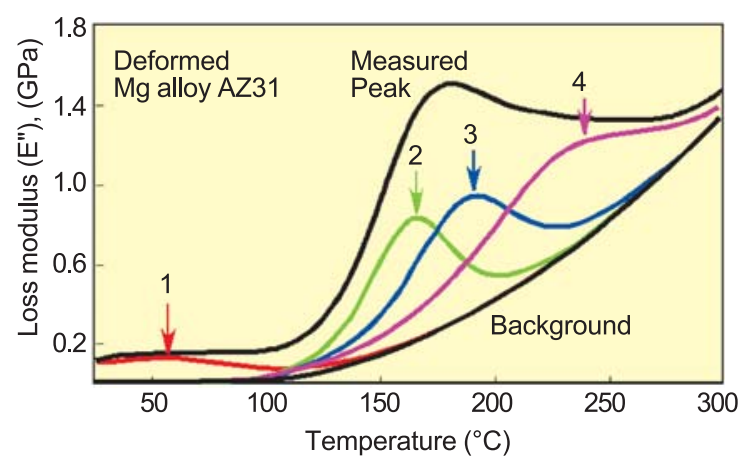

Figure 1: DMA peak in deformed Mg alloy AZ31. presented at industry workshops (USCAR Consortium, Deep Drawing Research Group) and at metallurgy and physics conferences (ASM, TMS, APS).

Also in FY 2004, Mg and Mg alloys were studied to determine how widespread this anomalous modulus behavior is and to gain additional clues as to its origin. $\mathrm{Mg}$ is an extremely lightweight HCP metal that would be a prime candidate for automotive applications if not for its poor formability and high chemical reactivity. Biaxial strain experiments did not produce a significant change in the elastic modulus, but the limited ductility prevented measurements at the high strains required to unambiguously reduce the modulus in $\mathrm{Al}$ alloys. Dynamic modulus analysis (DMA) found that plastic strain produced a broad peak in the imaginary component of the complex modulus. Previous researchers attributed this peak to grain boundary sliding, but plastic strain and twinning were found to be responsible for this peak which analysis indicated contains 4 subpeaks (Figure 1).

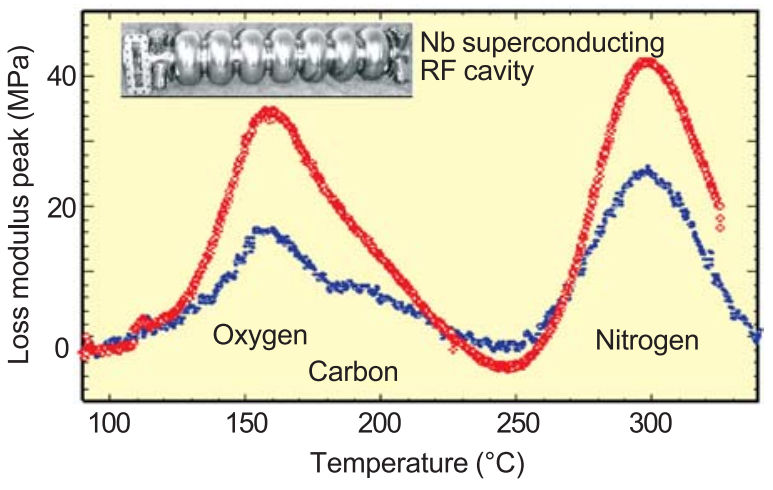

Figure 2: DMA peaks from two lots of $\mathrm{Nb}$ compared to the calculated locations for $O, C$, and $N$.

In a separate study, niobium is a BCC metal that DOE's Jefferson Laboratory (JL) uses to fabricate superconducting structures by stamping and forming operations similar to those used by the automotive industry to form BCC steel. The Jefferson Laboratory has funded NIST to investigate the catastrophic effect of impurities on the forming behavior of high purity $\mathrm{Nb}$. DMA experiments detected variations in the interstitial impurity content of this high purity metal (Figure 2), which will now be correlated with forming behavior.

\section{Contributors and Collaborators}

S.W. Banovic, R.J. Fields, L. Ma, D.J. Pitchure (Metallurgy Division, NIST); D. Dayan, A. Munitz (NRC-Negev); V. Luzin (NCNR); G. Myneni (JL); E. Chu (Alcoa); USCAR Springback Consortium (Alcoa, DaimlerChrysler, Ford, GM, LSTC, U.S. Steel) 


\section{Microstructural Origins of Surface Roughening and Strain Localizations}

The existing data, measurement methods, and basic understanding of metallurgical factors that influence friction, tearing, and surface finish during sheet metal fabrication are insufficient to meet the predictive modeling requirements of the automotive industry. This project addresses these needs by exploring the microstructural origins of the distribution of slip, surface roughening, and strain localization during plastic straining. The primary focus of these investigations is the relationships between the initial material characteristics and the deformation behavior of $\mathrm{Al}$ and $\mathrm{Fe}$ base sheet materials.

\section{Mark R. Stoudt and Stephen W. Banovic}

$\mathrm{R}$ eplacement of conventional steel sheet by aluminum alloys and high-strength low-alloy steels would significantly reduce automobile weight and increase fuel efficiency. However, wide spread application of these materials by the automotive industry is limited primarily due to formability issues and deficiencies in material databases/constitutive laws affecting finite element modeling. This project examines the underlying structure property relationships associated with formability in an attempt to improve the numerical simulations that predict deformation behavior of materials.

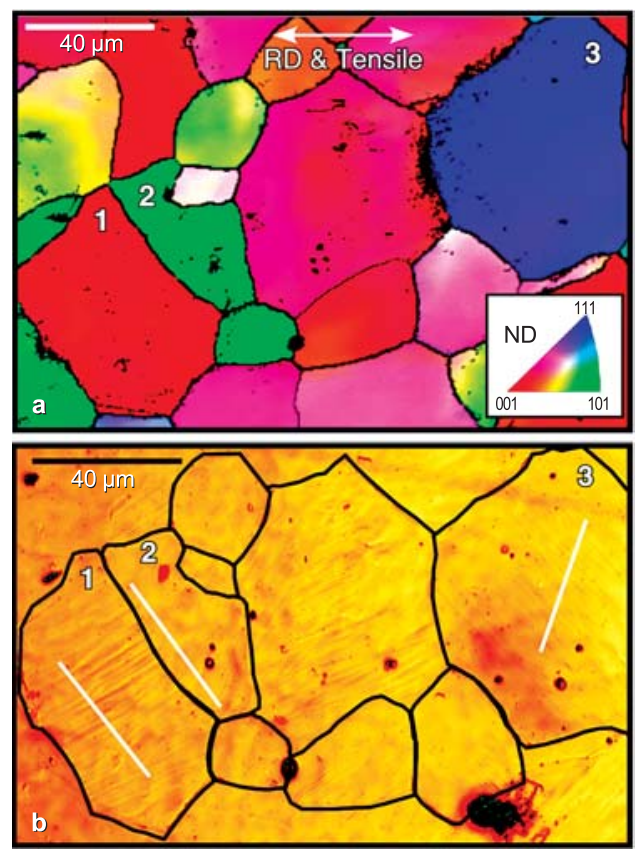

Figure 1: Surface Characterization of 6xxx series aluminum deformed to 0.08 uniaxial strain. a) EBSD generated grain orientation map; b) SLCM surface topograph of same region.
In FY 2004, several experimental techniques were used together to explore the relationships between strain, local crystallographic texture and surface roughening of aluminum alloys. Figure 1a is an electron backscattered diffraction (EBSD) map showing the variations in surface grain orientation on a $6 \times x x$ series aluminum sample after applying a uniaxial strain of 0.08 . As reflected in the figure inset, the surfaces of grains 1,2 and 3 have $<001>,<101>$ and $<111>$ orientations, respectively. A 3D topograph of the same region obtained with a scanning laser confocal microscope (SLCM) is shown directly below Figure 1a in Figure 1b. The labels indicate the same grains in both figures. There are clear differences in the slip band structures of the grains. The roughness profiles shown in Figure 2 were acquired from the SLCM data along the white lines in Figure 1b. The different crystallographic orientations in the three grains produced distinctly different roughness characters for the same level of macroscopic uniaxial strain. New statistical analysis techniques are currently being developed to investigate correlations in these data.

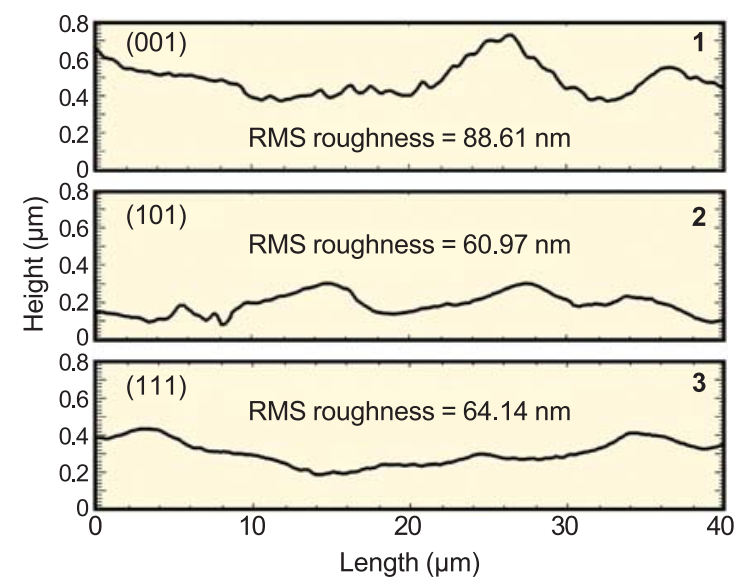

Figure 2: Roughness profiles obtained from the three individual grain orientations shown in Figure 1.

Other topics explored during this fiscal year include: 1) statistical analyses of surface roughness data aimed at determining what roughness measures would be most useful for industry; 2) EBSD/SLCM studies of AlMg binary alloys as a function of strain; and 3) exploratory work on new methods for measuring friction under conditions designed to emulate actual forming operations.

\section{Contributors and Collaborators}

J. Liu, T. Foecke, R.E. Ricker, L.E. Levine (Metallurgy Division, NIST); M.D. Vaudin (Ceramics Division, NIST); T. Gnäupel-Herold, (NIST Center for Neutron Research); J.B. Hubbard (CSTL, NIST); R. Reno, E. Moore (UMBC) 


\section{Plasticity, Fabrication Processes, and Performance}

\begin{abstract}
Predicting the final shape of a part produced using a complex fabrication process can be extremely challenging. This is particularly true when microstructure and residual stresses play significant roles. Understanding the interplay of these factors in industrially important test cases is the goal of this project. Work on this project was completed this fiscal year.
\end{abstract}

\section{Lyle E. Levine and Hank Prask (856)}

$\mathrm{D}$ iffraction provides a powerful means of very accurately measuring both microstructure and mechanical behavior in a way that provides insight simultaneously into both, and offers particular opportunities for understanding plasticity. This project collaborated with the developers of the NIST Object Oriented Finite Element (OOF) program to implement a plasticity model into OOF and devise critical experimental and computational benchmarks to validate OOF using the NCNR neutron diffractometer to measure residual stress, texture and elastic properties. Conventional X-ray sources were also used where stress states measured at or near surfaces were important for understanding the behavior of materials or to compare with predictions. Synchrotron X-rays were used to deal with subsurface residual stresses in the thin, highly textured samples that result from simulated forming operations. Specifically, the following tasks were part of this project:

- Experimentally validate calculation of the important role played by residual stresses on high-precision machining and forming operations (includes collaborative contributions from MEL, ALCOA and Boeing Corporation);

- Provide technical assistance and validation testing to CTCMS staff engaged in adding 3D plasticity to OOF;

- Develop the ability to characterize near-surface residual stresses; and

- Further develop neutron and synchrotron radiation sources to provide high-resolution data needed to relate microstructure, residual stresses, and their effect on springback.

\section{Overall Project Accomplishments}

- Validation of Distortion Prediction Method: In 2002, we validated residual stress measurement and

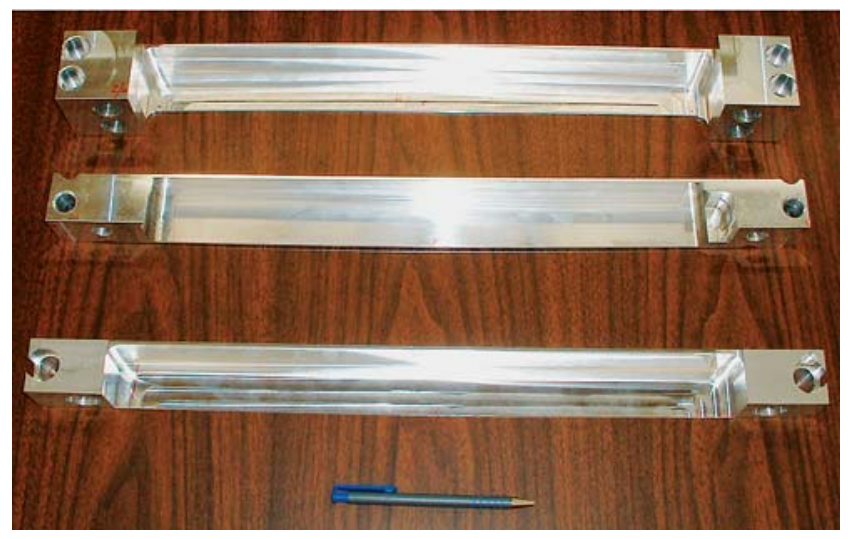

Figure 1: Aluminum parts machined to test shape changes caused by relaxation of residual stresses.

prediction methods used by industry. In 2003 and 2004, we used the NCNR to measure residual stress distributions within 7000 series aluminum bars and used high-speed machining to produce test parts. The shapes of the final parts were measured using a contact measuring machine and ALCOA will compare these shapes to their model predictions.

- Test of OOF: In FY 2003, textured polycrystalline samples were tested for elastic modulus dependence on texture. These results were compared with predictions using Electron Backscatter Diffraction and OOF.

- Measurement of residual stresses in standard springback cups in FY 2002: Measurements were provided for the project on Standard Tests and Data for Sheet Metal Formability. In addition, analyses were done to understand the origin of these stresses.

- Incorporation of plasticity: Project personnel worked with CTCMS staff to provide algorithms and techniques for incorporating plasticity into OOF.

Although this project is ending this fiscal year, outgrowths of the work on residual stress measurements of deformed sheet metal and plasticity modeling for OOF continue in other projects.

\section{Contributors and Collaborators}

R. Fields, R. deWit (Metallurgy Division, NIST); T. Gnäupel-Herold, V. Luzin (NIST Center for Neutron Research); R. Ivester, R. Polvani (Manufacturing Metrology Division, NIST); D. Bowden (Boeing); E. Chu (ALCOA); R. Reno (University of Maryland) 


\section{Standard Tests and Data for Sheet Metal Formability}

To meet the PNGV goals for fuel efficiency, the U.S. automotive industry is moving to lighter, high-strength materials for auto bodies. NIST has surveyed industry and found that providing designers with accurate material properties, and methods to incorporate them into finite element models of sheet metal forming dies, is a critical need for the US auto industry. This project seeks to develop new standard tests and metrology to accurately determine sheet metal mechanical response under forming conditions.

\section{Tim Foecke and Mark ladicola}

$F^{o}$ or the U.S. automotive industry to transition to new materials for formed sheet metal parts, they must be able to mechanically characterize the starting materials under realistic forming conditions, and input this information into die design models. The Metallurgy Division is conducting research to develop two sheet metal formability tests, along with associated metrology, that can be standardized and used by industry.

Springback is the elastic shape change to a part associated with the residual stresses that develop during the stamping process. This shape change complicates assembly and accurate fit-up; thus, the automotive industry has a strong desire to be able to either avoid it, or at least be able to predict its magnitude and design dies to account for it. The proposed test for springback consists of splitting open a ring cut from the sidewall of a deep drawn cup.

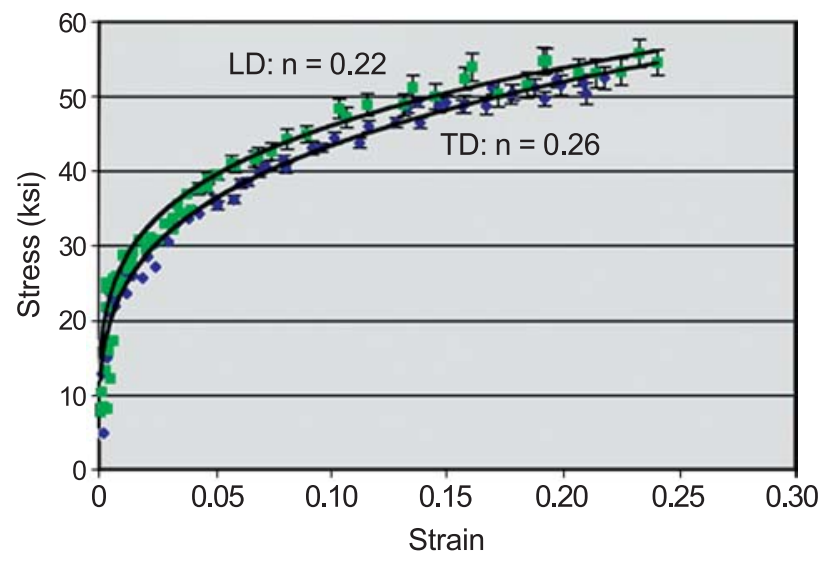

Figure 1: Balanced biaxial flow stress for 5182 Al alloy in transverse (TD) and rolling (LD) directions.
This year, the springback cup test was introduced to ASTM at its national meeting in Florida, leading to industry's proposal to form a new ASTM committee to explore standardization of this test. A crucial step in this process has been taken by identifying several industrial partners that are willing to participate. Additional participants from both the producer and end-user sides of the sheet metal industry are now being sought for this new committee. The initial robustness data set generated at NIST last year forms a solid basis for the design of a round-robin testing matrix to be implemented as part of the standardization process.

Last year, an x-ray stress measuring system was installed on the sheet metal formability station. This unique instrumentation allows for the direct, in situ measurement of the stress in a given direction while the sample is under multiaxial load. This year we made significant progress in mapping the multiaxial stress-strain surface for three aluminum sheet alloys of interest to the automotive industry: 5182, 6111 and 6022. Typical data is shown in the Figure 1, where for the first time the biaxial hardening exponent has been measured. In addition, both the flow stress and the hardening exponent, $n$, were found to differ in the transverse and rolling directions of the sheet. These data will be used by industry to eliminate several assumptions in place that extrapolate estimated biaxial flow behavior based on a series of uniaxial tensile tests. This should lead to more accurate designs and cost savings.

This year, NIST was asked to generate materials property data for simulations of prototype parts as part of the NUMISHEET 2005 conference. In addition, taking advantage of our unique capabilities to measure stress in a forming part, another layer of complexity of the prototype part simulation will require reproducing the measured stresses at points of the part under the forming load.

Plans for FY 2005 involve completing measurement of the multiaxial stress-strain surfaces for the three above-mentioned aluminum alloys, as well as for a series of steel alloys. This new project, in collaboration with modelers at GM, will involve studying how the flow surface evolves with different types and amounts of multiaxial prestrain.

\section{Contributors and Collaborators}

T. Gnäupel-Herold (NIST Center for Neutron Research); M. Shi (USS); E. Chu (ALCOA); C. Xia (Ford); T. Stoughton, M. Wenner, C.T. Wang (GM); A. Andersson (Volvo); E. Schedin (Avestapolarit) 


\section{The NIST Center for Theoretical and Computational Materials Science}

\begin{abstract}
The NIST Center for Theoretical and Computational Materials Science was founded in 1994 in order to fulfill its three-fold mission: to investigate important problems in materials theory and modeling with novel computational approaches; to create opportunities for collaboration where CTCMS can make a positive difference by virtue of its structure, focus, and people; and to develop powerful new tools for materials theory and modeling and accelerate their integration into industrial research. The CTCMS supports numerous materials theory and modeling projects both within and externally to the Materials Science and Engineering Laboratory.
\end{abstract}

James A. Warren and Benjamin P. Burton

$\mathrm{T}_{\mathrm{s}}^{\mathrm{h}}$ he NIST Center for Theoretical and Computational Materials Science (CTCMS) is a research program addressing industry needs for theory and modeling tools for materials design and processing. The CTCMS is a center of expertise in computational materials research that develops tools and techniques and fosters collaborations.

CTCMS integrates ongoing research at various institutions by forming temporary multidisciplinary and multi-institutional research teams as required to attack key materials issues of national importance. The CTCMS has three principal activities, all operating interactively: planning, research, and technology transfer. Workshops are held as the first step in defining technical research areas with significant technological impact, identifying team members, and building the infrastructure for collaborative research. The CTCMS provides infrastructure and support for its members, including an interactive World Wide Web server (www.ctems.nist.gov) and modern computing and workshop facilities.

Current research areas include theory and simulation of a wide range of materials behavior, including phase transformation kinetics and morphology, micromagnetics, composite materials, foams, microstructure and dynamics of disordered and partially ordered materials, complex fluids, materials reliability, reactive wetting, pattern formation, crystal growth, sintering, phase transitions in biological systems, and solidification. Current CTCMS working groups include the following:

- High-Throughput Analysis of Multicomponent Multiphase Diffusion Data.
- Phase Field Modeling Tools: The phase field method has become one of the most flexible and powerful methods for predicting the evolution of materials microstructure. This effort focuses on the development of both new applications for this method and tools enabling the solution of the complex equations which emerge from these models.

- Effective Hamiltonian Methods: Use of fundamental, quantum-mechanical descriptions to derive the properties of matter is the ultimate goal of materials modeling. This effort uses electronic structure calculations to derive the phase stability of alloys.

- WWW Tools for Scientific Collaboration: CTCMS is working with information science specialists to develop web-based tools for scientific collaboration.

- Tools for Neutron Scattering Measurements: While neutron scattering has become a critical tool for the probing of material structure and properties, interpretation of the results of experiments presents a host of challenges for the scientist. This effort attempts to develop a better theoretical framework for the interpretation of such experiments.

- Object-oriented finite element modeling of composite materials: This team of researchers is developing a set of object-oriented finite element modeling tools to improve the characterization and property prediction of composite materials. Public domain software tools are available at www.ctcms.nist.gov.

The CTCMS also hosts web pages with resources and tools in the following areas: an interactive, electronic library of Green's function and boundary element solution; accurate, standardized micromagnetics modeling tools; software tools to improve electronic packaging processes; and a tool to compute equilibrium crystal shapes. More detail can be found at www.ctems.nist.gov.

\section{Mechanisms for Collaboration with CTCMS}

The CTCMS facilitates numerous interactions between industry, academia, NIST, and other government and national labs to apply materials theory and modeling to solve U.S. industrial problems in materials design and processing. Researchers interested in joining existing efforts or starting new ones are encouraged to contact the CTCMS. The CTCMS participates in the National Research Council postdoctoral fellowship program and hosts short-term and long-term visitors. 


\section{Underlying Processes of Plastic Deformation in Metal Alloys}

\begin{abstract}
A substantial increase in the use of aluminum alloys and high-strength steels in automobiles would greatly increase fuel efficiency. The primary reason why this has not yet occurred is a lack of accurate deformation models for use in designing the stamping dies. This project is developing a physically based model of plastic deformation using a combination of statistical physics approaches, atomistic modeling and advanced measurement techniques.
\end{abstract}

\section{Lyle E. Levine}

$\mathrm{P}$ lastic deformation of metals (as in cold rolling, stamping, drawing, and metal fatigue) is of great importance to industries worldwide, and improvements in the basic technology would have a significant effect on the U.S. economy. Unfortunately, existing constitutive equations cannot accurately predict the material behavior, and many tryout and redesign steps are required. Another related difficulty is in the design of new alloys with improved formability characteristics. Currently, alloy design is done empirically with little understanding of how the various constituents affect the mechanical properties.

Addressing both of these issues, this project is focused on developing constitutive laws based upon the underlying physical processes that produce the observed mechanical behaviors in metal alloys. Such constitutive laws are inherently multi-scale since they must describe phenomena on length scales ranging from the atomistic all the way to the macroscopic stress-strain behavior of bulk material.

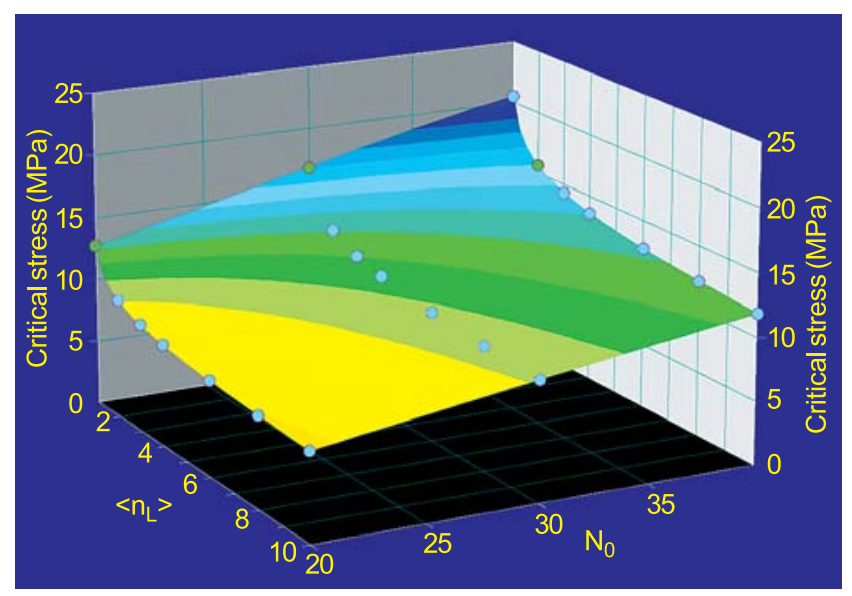

Figure 1: Universal flow surface (critical surface) for Al alloys at $\approx 15 \%$ strain.
Since the underlying processes are extremely complex, most of our theory, modeling and experimental efforts over the past several years were concentrated on single-crystal pure Al as a model system. This work culminated in the development of the segment length distribution (SLD) model, that describes how macroscopic deformation arises from the statistical behavior of large numbers of dislocations. The model correctly predicts the flow stress (Figure 1), the linear behavior of stage II hardening, the non-linear hardening in stage III and the development of slip lines and slip bands. The statistical variables, $<\mathrm{n}_{\mathrm{L}}>$ and $\mathrm{N}_{0}$, represent the density of primary dislocations and pinning points, respectively, in the sample. Alloying the $\mathrm{Al}$ changes the mechanical properties by affecting these statistical variables. Thus, this critical surface is a universal surface for all $\mathrm{Al}$ alloys. The next step is to connect the evolution of the statistical variables to the underlying physics for $\mathrm{Al}$ alloys using atomistic simulations that accurately incorporate the effects of alloy chemistry.

Quantum-mechanics based ( $a b$ initio) modeling is required to adequately describe the effects of chemistry in atomistic simulations. Unfortunately, such simulations are extremely CPU intensive and thereby limited to a few hundred atoms. Atomistic modeling using classical potentials can handle much larger systems but fails for large bond distortions and chemistry effects. A ground-breaking approach for directly coupling the two methods has been developed that allows the use of large-scale atomistic simulations with simple classical potentials while limiting the more accurate ab initio approaches to critical regions. Preliminary calculations of vacancy energies near dislocations are now underway.

Washington State University (WSU) is conducting experimental validation tests of the SLD model using chemi- and photo-emission techniques to study the time dependence of new surface production during deformation. NIST researchers designed the UHV tensile stage and single-crystal growth facilities, and WSU paid for the fabrication with funding from DOE.

Finally, NIST researchers are supporting the development of supporting science by serving on the executive committee of the international conference "Dislocations 2004" and coorganizing symposia at the Fall 2004 MRS meeting and at "Plasticity 2005."

\section{Contributors and Collaborators}

D. Pitchure, F. Tavazza (Metallurgy Division, NIST); A. Chaka (Physical \& Chemical Properties Division, NIST); T. Dickinson, S. Langford, M. Cai (WSU) 


\section{High Speed Machining}

\begin{abstract}
U.S. industry annually spends $\$ 200$ billion on the machining of metal parts, and potential cost savings are a driver towards high-speed machining. Traditional knowledge-based approaches have not been effective in improving the efficiency of this rapidly developing field: One recent study showed that industry chose the correct machining parameters less than half of the time. Through this project, NIST will provide measurement capabilities, materials data, and assessment of constitutive laws and deformation models, which will provide users with a capability of producing a first part correct.
\end{abstract}

\section{Carelyn E. Campbell}

$\mathrm{T}$ o improve the efficiency of high-speed machining and tooling, industry has employed finite element modeling (FEM) to predict correct tooling and machining parameters. However, FEM has had limited success as much of the needed material property data for high speed machining processes, which involve large strains $(>>1)$, high-strain rates (up to $10^{6} \mathrm{~s}^{-1}$ ), and high-heating rates (greater than $10^{5} \mathrm{C} / \mathrm{s}$ ), is insufficient. This project emphasizes two complementary areas: (1) the development of fundamental data on dynamic material behavior at high strain and heating rates and the assessment of the constitutive laws used to model this behavior; (2) the modeling and characterization of the microstructural changes that occur during the rapid deformation and rapid heating in real machining operations. Residual stresses, responsible for part distortion after machining, are being measured at the NIST Center for Neutron Research.

The first research effort is focused on obtaining the materials data and assessing whether currently used constitutive laws are appropriate. A dynamic material testing facility using a pulse-heated Kolsky bar has been developed at NIST and is being used to measure dynamic stress-strain data at high-strain rates (approximately $500 \mathrm{~s}^{-1}$ to $10^{5} \mathrm{~s}^{-1}$ ) with heating rates ranging from $10^{3} \mathrm{Ks}^{-1}$ to $1 \mathrm{Ks}^{-1}$. Measurements on 1045 steel showed significant differences in the stress-strain behavior depending on the heating and strain rates (Figure 1). The two microstructures shown in Figure 1 demonstrate the observed microstructural differences resulting from different heating rates. Test 504 was rapidly heated and quenched, leaving no time for the pearlite to dissolve and reform. In contrast, test 509 was heated, held at temperature for $1.4 \mathrm{~s}$, then quenched, leaving enough time for the pearlite to dissolve and then re-crystallize during quenching, producing a finer grain size.

These results demonstrated that the non-time dependent constitutive relationships used by industry to describe the stress-strain data for machining simulations are not correct. A time-dependence must be included in the constitutive relationship to correctly model the material behavior during high-speed machining. Using the dynamic material property data collected using the pulse-heated Kolsky bar, time-dependent constitutive laws will be developed and then tested in machining simulations.

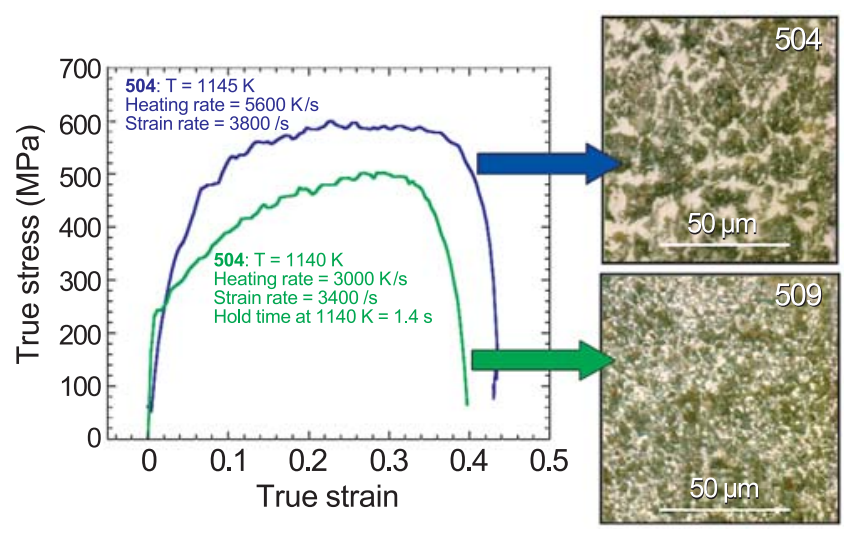

Figure 1: The effect of various heating and strain rates on both the stress-strain behavior and the microstructure of 1045 steel.

The second area of research focuses on characterizing and modeling microstructural changes in the deformation/hot zone of aluminum alloys during machining. Transmission electron microscopy (TEM) correlated with optical microscopy of the chip has revealed bands of re-crystallized grains. The work surface is being characterized using microhardness measurements, TEM, optical microscopy and residual stress measurements. A model based on these microstructural characterizations and temperature $v s$. time data from thermal images will be used as input to a microstructure model. The microstructure model, along with the time-dependent constitutive relationship, will be implemented into a finite element code for machining simulation.

\section{Contributors and Collaborators}

D. Basak, L. Levine, R. Fields, R. deWit, W. Boettinger, L. Bendersky, T. Gnäupel-Herold, H. Prask (NIST/MSEL); R. Ivester, R. Rhorer, K. Jurrens, J. Soons, R. Polvani, E. Whitenton, M. Kennedy, B. Dutterer, G. Blessing (Manufacturing Engineering Laboratory, NIST); T. Burns (Information Technology Laboratory, NIST); H. Yoon (PL); M. Davies (U. North Carolina-Charlotte) 


\section{Mechanisms for Delivery of Thermodynamic and Kinetic Data}

The availability of reliable materials data is the key to successful design of new materials and manufacturing processes. Many commercial processes are controlled by the thermodynamic and diffusion properties of the material. Thermodynamic and diffusion mobility databases provide an efficient method of storing the wealth of these data, and software tools allow the user to efficiently retrieve the needed information.

\section{Ursula R. Kattner and Carelyn E. Campbell}

T he complexity of traditional thermodynamics and diffusion kinetics prevented their direct application to the design of complex materials and processes in the past. By the same token, graphical representation of multicomponent systems is too complex, and storage of every single datum is inefficient due to the enormous amount of data. However, mathematical functions that represent thermodynamic and diffusion properties of the phases permit efficient storage. Since these functions are based on physical models, they further provide the power of extrapolation of binary and ternary systems to higher order systems. Software then allows calculation of the desired quantities. This approach is called the CALPHAD methodology. Unfortunately, previous software was tailored to be efficient for expert users and was difficult for the occasional, less-experienced user.

The databases and software developed in this project are designed to provide users with simple tools to retrieve and disseminate information needed for efficient materials and process design.

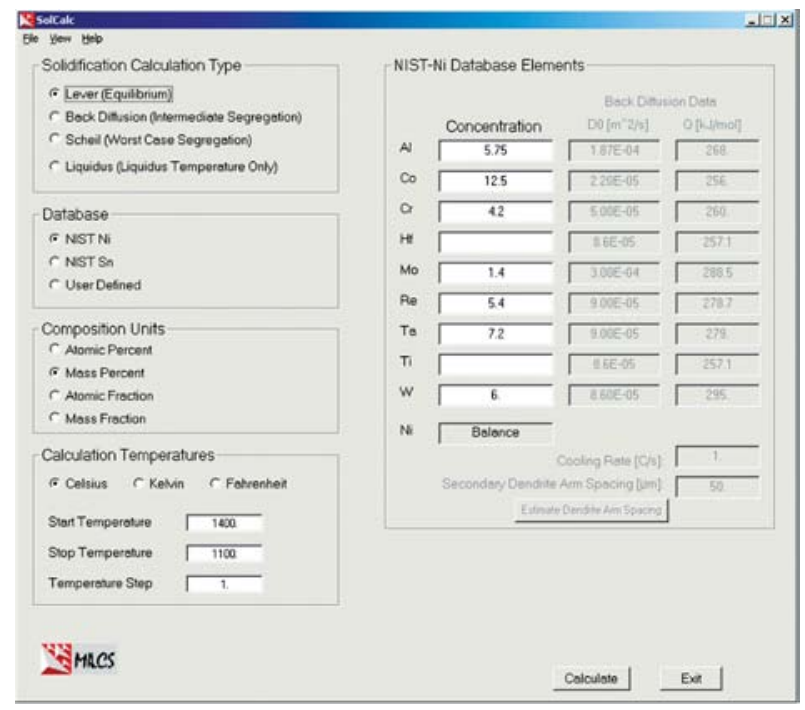

Figure 1: Graphical user interface for solidification calculations.
A modern, user-friendly front end for user input has been developed for solidification calculations (Figure 1). The results of the calculation are delivered to the user in graphical as well as in tabular form. This program is bundled with the NIST superalloy and solder thermodynamic databases, but has the capability to work with user-supplied databases as well. Results can be used with a Mathematica script for DTA analysis simulation. The software, databases and scripts are available on the Metallurgy Division website (www.metallurgy.nist.gov).

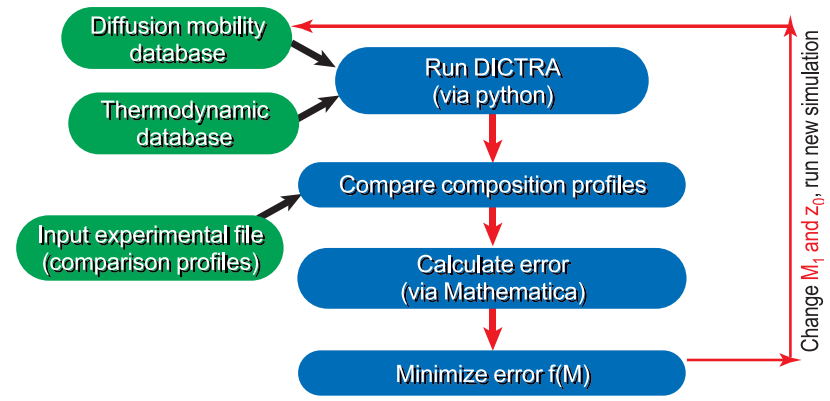

Figure 2: Schematic of an optimization scheme to allow direct input of diffusion couple composition profiles.

Multicomponent, multiphase diffusion couples can be simulated using NIST thermodynamic and diffusion mobility databases, and experimental and calculated composition profiles can be compared. However, the complexity of such couples prevents experimental diffusion coefficients from being easily extracted. Current work focuses on methods to allow experimental composition profiles to be directly inputted into a data assessment process, so the composition profiles can be directly related to the diffusion mobility parameters stored in the database (Figure 2).

Interactions developed through the "High Throughput Analysis of Multicomponent Multiphase Diffusion" workshop series have led to several software codes aimed to improve the analysis of multicomponent diffusion couples based on combinatorial libraries. These codes and other resources are listed on the group's website (www.ctems.nist.gov).

Work continues to convert the NIST Diffusion Data Center into an electronic searchable form. A preliminary version should be available by December 2004 .

\section{Contributors and Collaborators}

W.J. Boettinger (Metallurgy Division, NIST); A.R. Roosen (Center for Theoretical and Computational Materials Science, NIST); J.-C. Zhao (General Electric); L. Höglund (Thermo-Calc AB) 


\section{Phase Field Modeling of Materials Systems: Simulation of Polycrystalline Growth}

\begin{abstract}
Many properties of structural and functional materials depend on the distribution of composition, phases, grain orientations, internal surface stresses, and microstructure. These structures span length scales from nanometers to meters. Predictive models are needed to reduce the enormous costs and development times involved in designing and inserting new materials into products. Phase field models of solidification, coupled to stress, grain orientation, and solute and vacancy dynamics are being developed to address these needs.
\end{abstract}

\section{James A. Warren}

$\mathrm{M}$ odeling of microstructures produced by solidification, grain evolution, and stress, as well as other processes, involves mathematical solution of equations for heat flow, fluid flow, current flow and/or solute diffusion. Boundary conditions on external surfaces reflect the macroscopic processing conditions, while boundary conditions at internal interfaces correspond to the liquid crystal (grain) or grain-grain interfaces. These internal interfaces are moving boundaries and require boundary conditions with thermodynamic and kinetic character.

To deal with the complex interfacial shapes that develop during solidification, grain growth, and the application of external stress or pressures, the phase field method has become the technique of choice for computational materials scientists. This approach often requires numerical techniques to solve the model equations but readily deals with complex interface shapes and topological changes. The research, conducted in collaboration with the Polymers Division, is also supported by the NIST Center for Theoretical and Computational Materials Science.

This year, significant advances were made in the understanding of the formation of multi-grained (polycrystalline) materials. Polycrystals often arise by either the impingement and growth of grains nucleated in a liquid (equiaxed grains), or via the nucleation of columnar grains on a surface. In collaboration with researchers in Japan and Hungary, we developed a phase field model to study such growth. In a surprising development, this same model has now elucidated a third mechanism of polycrystalline growth: growth front nucleation. This mode of growth is manifested when new orientations nucleate on the front of a growing crystal, yielding a densely branched morphology in which the structural symmetries that arise due to effects of surface energy anisotropy are disrupted by the nucleation process, yielding an isotropic pattern. This model demonstrated that this type of growth can be initiated by either static or dynamic heterogeneities in the solidifying system, solving a riddle that has been of substantial interest to materials scientists. This work, published in Nature Materials, has attracted extensive academic and industrial interest.

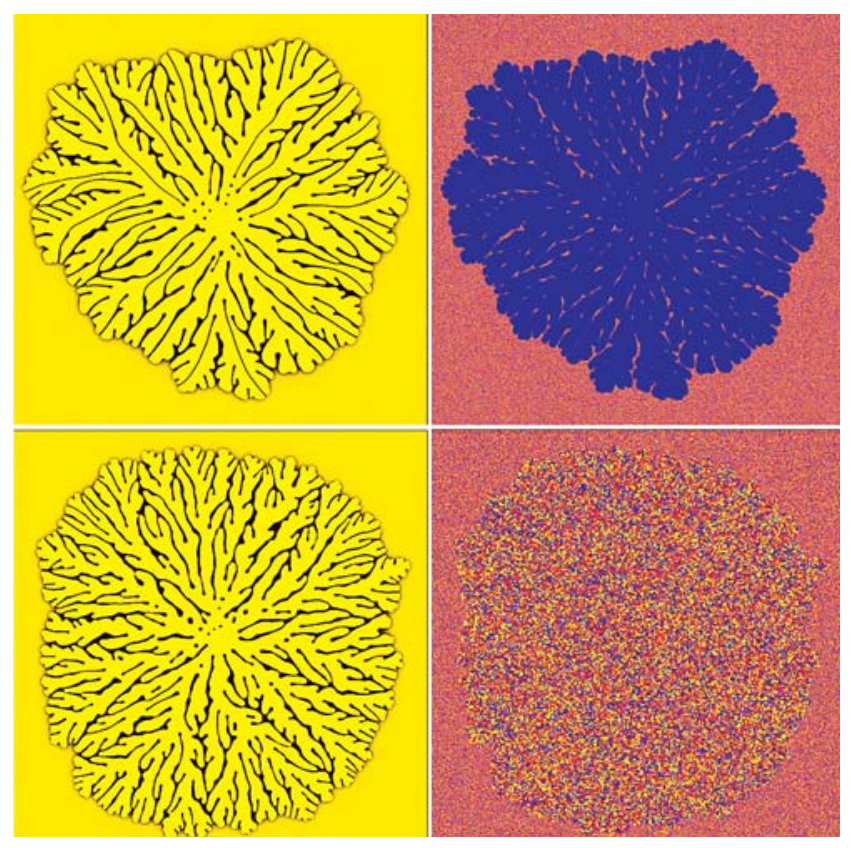

Figure 1: Two simulations of seaweed-like growth of an alloy. The left column shows the solute profile while the right column shows grain orientation. The upper row is a single crystal while the lower is polycrystalline. Using such techniques, the potential for polycrystalline growth to disrupt the influence of surface energy anisotropy (which gives dendritic growth) is demonstrated.

Although a remarkable amount of progress has been made, there are still physical phenomena that need to be properly included in phase field models if engineering impact is desired. With this in mind, much effort has been devoted to account for the effects of stress and vacancies on both solidification and interdiffusion. It is also believed that our model for polycrystalline growth may explain spherulitic growth as well as provide insight into the observations of Bendersky and NIST coworkers of a stable glass phase in highly supercooled alloys.

\section{Contributors and Collaborators}

W. Boettinger, J. Guyer, D. Wheeler (Metallurgy Division, NIST); J. Douglas (Polymers Division, NIST); L. Granasy, T. Pusztai (RISSPO, Hungary) 


\section{Hardness Standardization: Rockwell, Vickers, Knoop}

Hardness is the primary test measurement used to determine and specify the mechanical properties of metal products. The Metallurgy Division is engaged in all levels of standards activities to assist U.S. industry in making hardness measurements compatible with other countries around the world. These activities include the standardization of the national hardness scales, development of primary reference transfer standards, leadership in national and international standards writing organizations, and interactions and comparisons with U.S. laboratories and the National Metrology Institutes of other countries.

\section{Carlos Beauchamp and Sam Low}

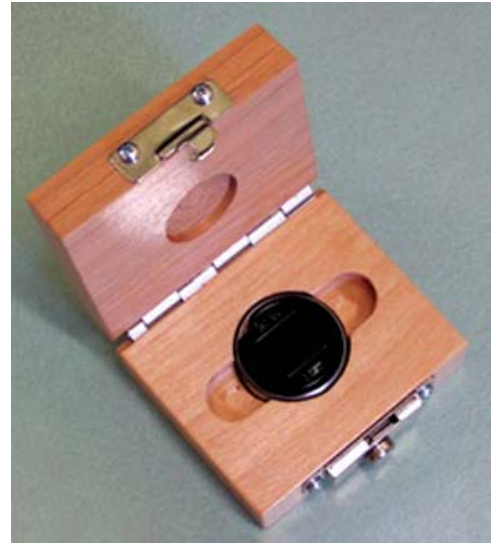

Figure 1: Prototype of new steel microhardness SRM.

\begin{abstract}
A the international level, we are leading the Working Group on Hardness (WGH) under the International Committee for Weights and Measures (CIPM). The primary goal is to standardize hardness measurements worldwide. As secretary of the WGH, we have led an effort this year to better define the Rockwell hardness test procedure used by National Metrology Institutes (NMIs). Other activities include chairing the ASTM-International committee on Indentation Hardness Testing and heading the U.S. delegation to the ISO committee on hardness testing of metals, which oversees the development of the respective hardness test method standards published by those organizations; and as the Secretariat for the International Organization of Legal Metrology (OIML) committee on hardness, we have revised the international requirements for regulating Rockwell hardness machines.
\end{abstract}

The primary task at the national level is to standardize the U.S. national hardness scales and to provide a means of transferring these scale values to industry. Currently, we are producing test block Standard Reference Materials ${ }^{\circledR}$ (SRMs) for the Rockwell, Vickers, and Knoop hardness scales, as well as developing new reference standards. Twelve different microhardness SRMs for Vickers and Knoop hardness are now available. Fifteen units of a new steel SRM are in the process of being certified at a nominal hardness of $760 \mathrm{kgf} / \mathrm{mm}^{2}$ for each of the Vickers and Knoop hardness scales. These SRMs are being certified at loads of $2.943 \mathrm{~N}, 4.905 \mathrm{~N}$, and $9.81 \mathrm{~N}$. In addition, renewal of low inventory of two of the Knoop Scale SRMs is underway. Ten units of each of the copper and nickel SRMs are being certified with nominal hardness levels of $125 \mathrm{kgf} / \mathrm{mm}^{2}$ and
$600 \mathrm{kgf} / \mathrm{mm}^{2}$, respectively, at loads of $0.245 \mathrm{~N}$, $0.490 \mathrm{~N}$, and $0.981 \mathrm{~N}$.

We also introduced two new SRMs for the Rockwell B scale (HRB) to complement the three SRM Rockwell C scale blocks currently available. The HRB scale is used for testing softer metals, such as aluminum, copper and brass. Work is also continuing to produce a new Rockwell hardness diamond indenter SRM, which has become feasible due to the successful completion of a Small Business Innovative Research (SBIR) Phase-2 project with Gilmore Diamond Tools to develop an improved method for manufacturing geometrically correct Rockwell diamond indenters.

Other activities at the national level occurring this year included: the assessment of commercial secondary hardness calibration laboratories for the NIST National Voluntary Laboratory Accreditation Program (NVLAP) providing direct linkage to the use of the NIST SRMs; the development of a NVLAP proficiency testing program for hardness calibration laboratories; and the development of indentation Finite Element Analysis models that have been used to analyze the effect of using different indenter materials for Rockwell hardness tests in support of proposed revisions to international test standards.

\section{Contributors and Collaborators}

C. Johnson, J. Fink, D. Kelley, L. Ma, H. Gates (Metallurgy Division, NIST); J. Song (Manufacturing Engineering Laboratory, NIST); W. Liggett, Jr., N. Zhang (Information Technology Laboratory, NIST); S. Doty, B. Belzer, D. Faison (National Voluntary Laboratory Accreditation Program, NIST); W. Stiefel (Technology Services, NIST); M. Mihalec (Gilmore Diamond Tools, Providence, RI) 


\section{Nanometrology}

The burgeoning field of nanomaterials extends across the full range of traditional material classes, including all forms of metals, polymers, and ceramics. No previous materials technology has shown so prodigiously a potential for concurrent advances in research and industry as does the field of nanomaterials in mechanical devices, electronic, magnetic, and optical components, quantum computing, tissue engineering and other biotechnologies, and as-yet unanticipated exploitations of as-yet undiscovered novel properties of nanoscale assemblies of particles. Already, there is growing excitement surrounding the ability of some molecules or particles to self-assemble at the nanoscale to form new materials with unusual properties. Nanometrology, i.e., the ability to conduct measurements at these dimensions, to characterize the materials, and to elucidate the structure and nature of these new and novel assemblies, is a requisite and fundamental cornerstone that must be established securely if this technology is to flourish.

NIST is uniquely positioned to lead the development of the measurement methods, instrumentation, standards, and reference materials that, together, will form the metrological infrastructure essential to the success of nanotechnology.

The MSEL Nanometrology Program incorporates basic measurement metrologies to determine material properties, process monitoring at the nanoscale, nanomanufacturing and fabrication techniques, and structural characterization and analysis techniques such as advanced imaging and multiscale modeling. The Program comprises 22 projects in the Ceramics, Materials Reliability, Metallurgy, and Polymers Divisions, and includes structural characterization using neutron scattering at the NIST Center for Neutron Research (NCNR). The projects cover a wide range of measurement and characterization methods grouped into the areas of mechanical property measurement, chemical and structural characterization and imaging, fabrication and monitoring of nanoprocesses and events, and modeling of nanoscale properties. In each area, we work to advance basic measurement capabilities and lead the intercomparison, standardization, and calibration of test methods. The newly completed Advanced Measurement Laboratory at the NIST Gaithersburg site provides an incomparable environment for accurate nanoscale metrology.

In the area of mechanical property measurement, we are developing and standardizing techniques for determining nanoscale elastic properties (elastic moduli, Poison's ratio, and internal stress), plastic deformation, density, adhesion, friction, stiction, and tribological behavior. Work in nanoindentation, used extensively in determining mechanical properties of thin films and nanostructures, focuses on developing traceable calibration methodologies and standard test methods. We also use atomic force acoustic microscopy, surface acoustic wave spectroscopy, and Brillouin light scattering to measure the mechanical properties of thin films. In addition, we are developing micro- and nano-scale structures and test methods to measure strength and fracture behavior of interfaces and materials having very small volumes.

The chemical and structural characterization and imaging utilize neutron and $\mathrm{x}$-ray beam lines at three facilities: the NCNR; the National Synchrotron Light Source at Brookhaven National Laboratory; and the Advanced Photon Source at Argonne National Laboratory. Innovative scattering and spectroscopy methods are advancing our ability to obtain a wide range of chemical and structural information at the nanoscale, including chemical bond identification and orientation, polyelectrolyte dynamics, and equilibrium structures. In collaboration with three other NIST laboratories, we are developing electron microscopy and spectroscopy instrumentation for quantitative, $3 \mathrm{D}$ chemical imaging at the nanoscale. Other characterization projects include work on gradient reference specimens for the calibration of advanced scanning probe microscopy, and the application of carbon nanotubes as physical probes of cell membranes.

Efforts in the fabrication and monitoring of nanoscale processes and events include the study of electrochemical and microfluidic methods for fabricating nanostructures, novel approaches to nanocalorimetry for the study of interfacial reactions, in situ observations of nanoparticle and nanotube dispersion and alignment, and advanced instrumentation for nanotribology experiments.

Finally, we have extensive efforts in the theory, modeling, and prediction of material properties and behavior extending from nanoscale to macroscale dimensions. Modeling efforts include large-scale finite element methods, multiscale Green's function methods, classical atomistic simulations, first principles, and quantum mechanical calculations using density functional theory. Often, several modeling methods must be combined into one study to accurately describe the material behavior; thus, we pay great attention to the correct interfacing between models operating at different length scales, to ensure that our models properly capture the physics of both components and total systems.

Contact: Gery R. Stafford or John E. Bonevich (Metallurgy Division) 


\section{Nanoscale Characterization by Electron Microscopy}

\begin{abstract}
Electron microscopy is used to characterize the structure and composition of materials at the nanometer scale to better understand and improve their properties. New measurement techniques in electron microscopy are being developed and applied to materials science research. The MSEL Electron Microscopy Facility primarily serves the Metallurgy, Ceramics, and Polymers Divisions as well as other NIST staff and outside collaborative research efforts.
\end{abstract}

\section{John E. Bonevich}

The MSEL Electron Microscopy Facility consists of two transmission electron microscopes, three scanning electron microscopes, a specimen preparation laboratory, and an image analysis/computational laboratory. The JEM3010 TEM can resolve atomic structures and employs an energy selecting imaging filter (IF) and X-ray detector (EDS) for analytical characterization of thin foil specimens. The S-4700-II FE-SEM employs electron backscattered diffraction/phase identification (EBSD) and EDS systems to characterize the crystallographic texture and composition of materials.

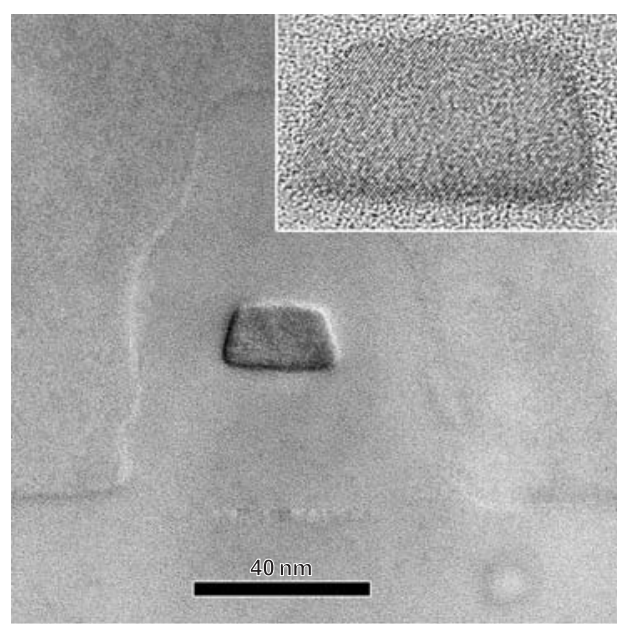

Figure 2: Confined Si single electron transistor device.

Highlights from the EM Facility for FY2004 include:

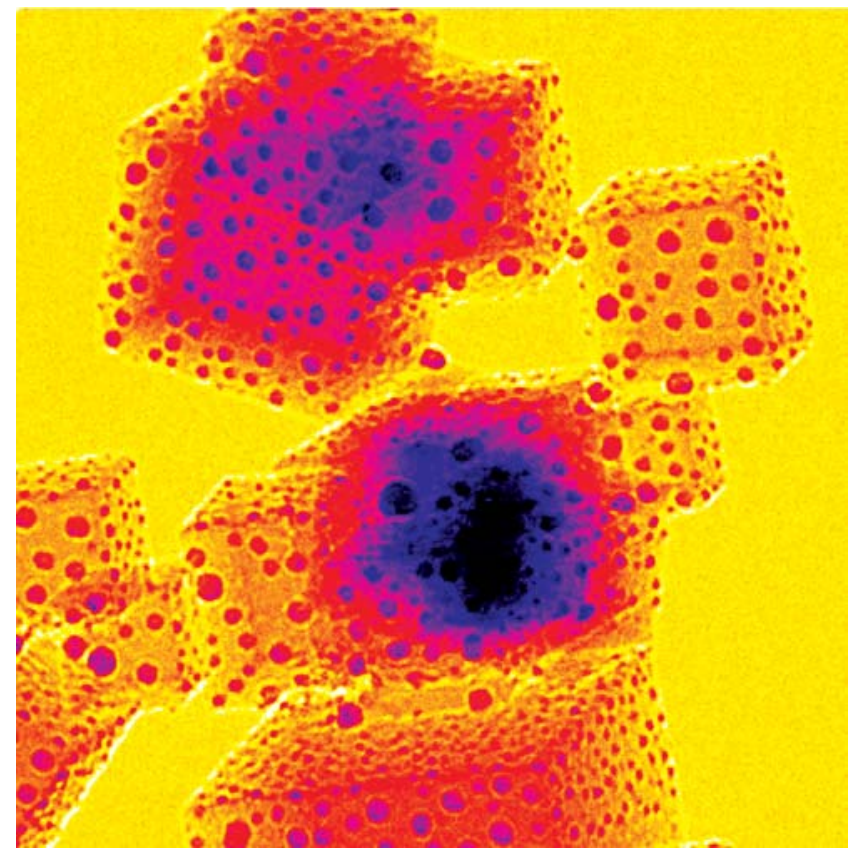

Figure 1: MgO cubes with surfaces decorated by small gold particles. These particle ensembles are used in model studies of $3 D$ chemical imaging at the nanoscale (electron tomography).

\begin{abstract}
tomic-scale structure and compositional insights to the control of their properties. For instance, direct observation of local structures by transmission electron microscopy (TEM) provides important feedback to the optimization of crystal growth and techniques. Various characteristics may be size and morphology, defects such as stacking faults, twins, grain boundaries and second phase particles. as well as the atomic structure of surfaces and interfaces, can provide powerful knowledge for engineering and understanding materials.
\end{abstract}

- A new, high-sensitivity EBSD CCD camera which acquires in excess of 70 patterns/second, was installed on the FE-SEM.

- 3D Chemical Imaging at the Nanoscale, a collaborative project with CSTL and PL on tomographic characterization of materials, was initiated (Figure 1).

- Research collaboration with the Semiconductor Electronics and Electricity Divisions (EEEL) has characterized quantum effects in confined Si devices (Figure 2).

- Sub-100 nm Ag interconnects formed by superconformal electrochemical deposition was characterized.

\section{Contributors and Collaborators}

D. Josell, T. Moffat, L. Bendersky (Metallurgy Division, NIST); I. Levin, B. Hockey (Ceramics Division, NIST); J.H.J. Scott (Surfaces \& Microanalysis Science Division, NIST); Z. Levine (Optoelectronics Division, NIST); E. Vogel (Semiconductor Electronics Division, NIST) 


\section{Nanostructure Fabrication Processes: Patterned Electrodeposition by Surfactant-Mediated Growth}

Novel structures and devices can be formed through template electrodeposition. The ultimate pattern resolution is determined by the size and packing of molecules that comprise the template and their ability to inhibit or catalyze various electrochemical reactions. In the past year, the effect of molecular functionality on the metal deposition process has been explored.

\section{Thomas P. Moffat and Michael J. Fasolka}

$\mathrm{O}$ ver the last decade, electrochemical processing has been undergoing a renaissance with the fabrication of new materials and novel microstructures. The development of new measurement techniques and metrological tools for studying controlled growth at fine length scales is the primary objective of this effort.

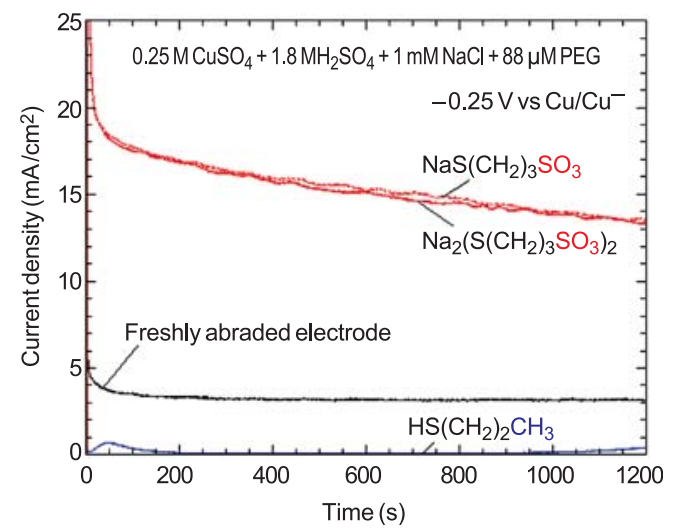

Figure 1: Influence of small changes in molecular structure, i.e., $\mathrm{SO}_{3}^{-}$vs. $\mathrm{CH}_{3}$, on the rate of copper deposition.

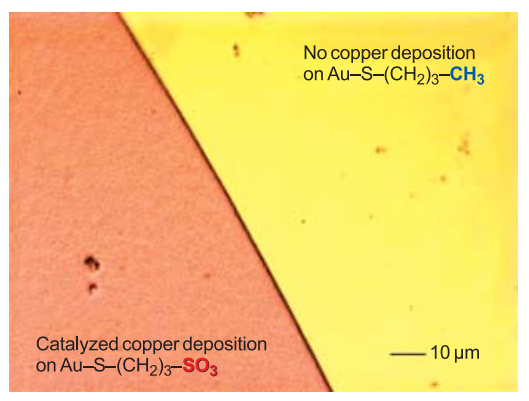

Figure 2: Copper deposition on an alknethiol derivatized gold substrate only occurs in regions bearing $-\mathrm{SO}_{3}^{-}$terminated molecules.

The three key metrology issues addressed are:

1. The rate differentiation accessible via surfactant mediated metal film growth;
2. The role of molecular functionality of the surfactant and robust design rules; and

3. The anisotropy induced in the electrocrystallization reaction rate for a given surfactant.

As an example, an alkanethiol monolayer film can either block or accelerate metal deposition depending on the molecular functionality of its terminal group. A dynamic range spanning several orders of magnitude is possible depending on the system in question (Figures 1 and 2). Based on these measurements, selective metal deposition may be obtained by patterning a substrate with the appropriate molecule.
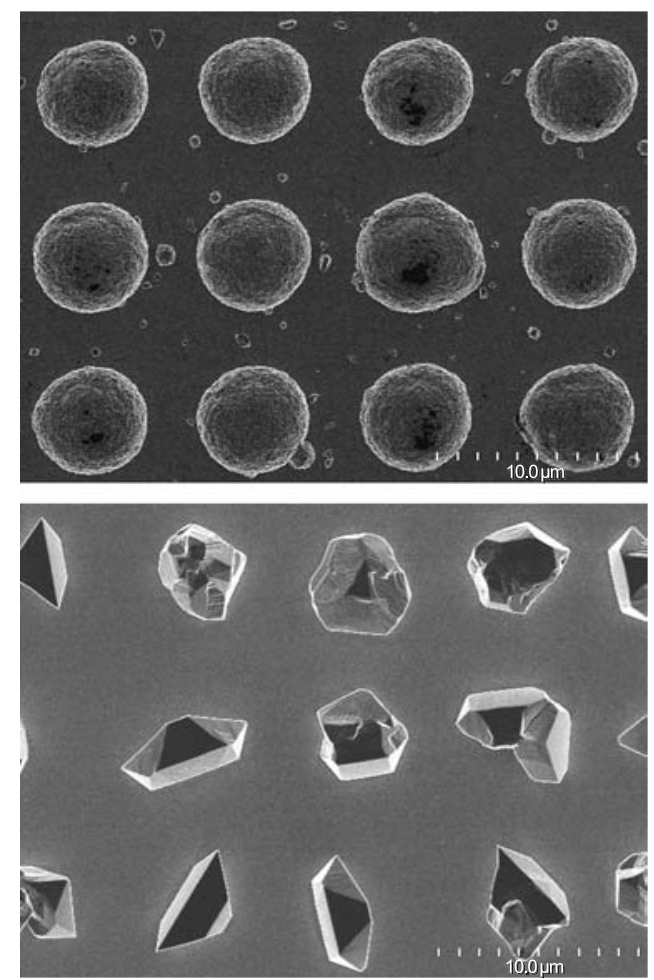

Figure 3: Spatially patterned monolayer films used to control nucleation and anisotropic growth of copper crystals.

Through contact printing, higher resolution patterning is possible, along with some interesting opportunities for high-throughput combinatorial research. By varying the surface chemistry, growth of isotropic or faceted crystals has been shown to be possible (Figure 3).

\section{Contributors and Collaborators}

D. Josell, J. Mallett, W.F. Egelhoff (Metallurgy Division, NIST); M. Walker, L. Richter (CSTL, NIST) 


\section{Nanostructure Fabrication Processes: Thin Film Stress Measurements}

The microelectronics community uses electrodeposition to produce solderable surface finishes, magnetic recording media, and copper interconnections in printed circuit boards and integrated circuits. These films tend to develop sizable mechanical stresses that can lead to loss of adhesion and the generation of bulk and surface defects. This project focuses on the measurement of these stresses which should enable the development of effective mitigation strategies.

\section{Gery R. Stafford and Ole Kongstein}

$\mathrm{E}$ lectrodeposited films tend to develop sizable mechanical stresses during deposition due to the nucleation and growth process or solution additives and alloying elements. We have established an optical bench dedicated to the in situ measurement of growth and residual stress during electrodeposition using the wafer curvature method. In one approach, a substrate of borosilicate glass is evaporated with $250 \mathrm{~nm}$ of gold. The force exerted on the cantilever by the electrodeposit causes curvature of the cantilever, which is monitored during electrodeposition. Forces on the order of $0.03 \mathrm{~N} / \mathrm{m}$ can be resolved during film deposition.

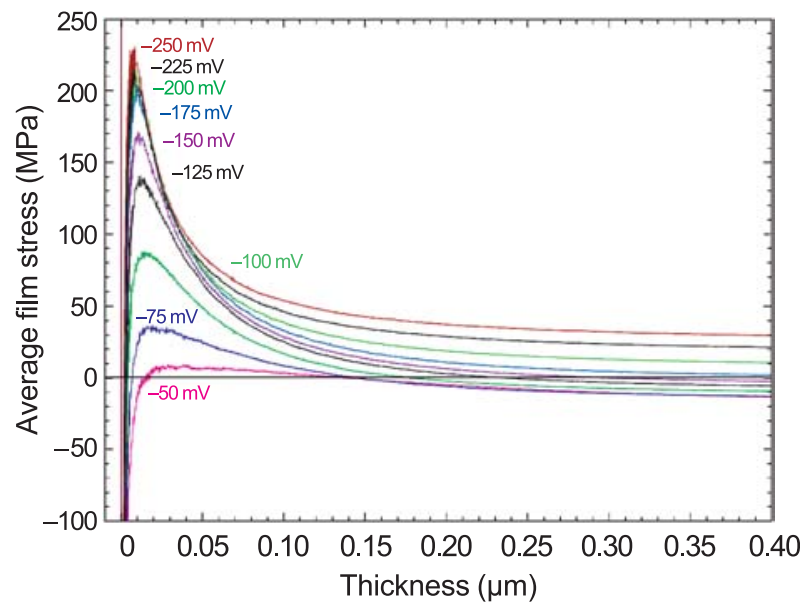

Figure 1: Average film stress during copper deposition.

Figure 1 shows the average in-plane stress associated with the deposition of copper from an additive-free sulfate electrolyte. The rapid rise in tensile stress (within the first $20 \mathrm{~nm}$ ) and its dependence on deposition potential are consistent with nuclei coalescence and grain boundary formation. The highest tensile stresses are associated with high nucleation densities which are obtained at more negative deposition potentials. As the deposit thickens, the average film stress decreases and becomes compressive in deposits formed at small deposition overpotentials. In the physical vapor deposition literature, this compressive stress has been attributed to the non-equilibrium concentration of mobile ad-atoms on the surface that are driven into the grain boundaries.

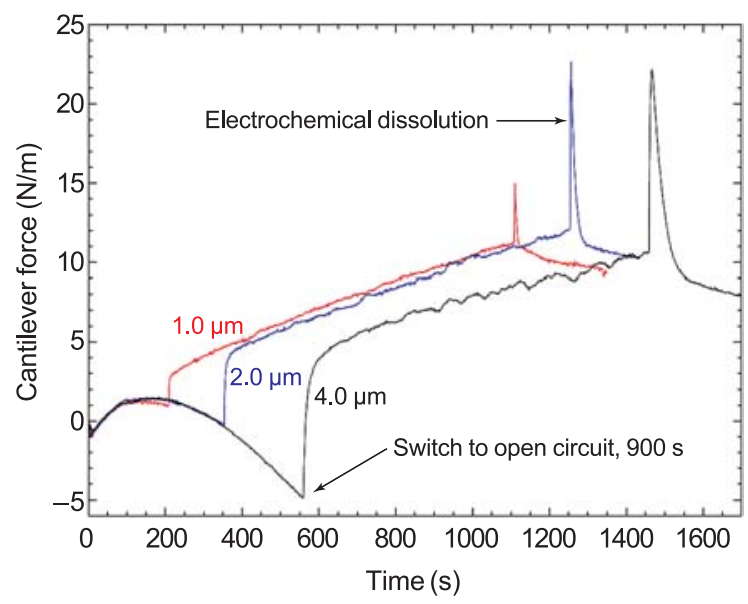

Figure 2: Force/width exerted on the cantilever during the electrochemical processing of Sn on $\mathrm{Cu}$.

We have also examined the force exerted onto a copper cantilever electrode during the deposition of matte tin (Sn), Figure 2. This is a particularly important system since the growth of tin whiskers, known to produce electrical short circuits and device failure, has been attributed to both the residual stress in the electrodeposit as well as that generated from intermetallic formation at the $\mathrm{Sn}-\mathrm{Cu}$ interface. The force curves show the following features: a tensile to compressive transition during deposition, a significant tensile relaxation when plating is discontinued, the development of tensile stress while the deposit is at open circuit, and a residual tensile stress after the deposit is electrochemically dissolved. The two latter features are attributed to the formation of the $\mathrm{Cu}_{6} \mathrm{Sn}_{5}$ intermetallic (IMC) at the $\mathrm{Sn}-\mathrm{Cu}$ interface. A tensile stress is generated since the IMC has approximately $6 \%$ smaller volume than a rule of mixture combination of the $\mathrm{Sn}$ and $\mathrm{Cu}$ reactants. Calculating the IMC thickness from the difference in Sn deposited and stripped, a nominal stress in the intermetallic was estimated to be $1.2 \mathrm{GPa}$ after only one hour. Future work will focus on determining to what degree this increases the compressive stress in the Sn electrodeposit.

\section{Contributors and Collaborators}

W. Boettinger, U. Bertocci (Metallurgy Division, NIST) 


\section{Nanomechanics: Coupling Modeling with Experiments}

\begin{abstract}
Knowledge of mechanical behavior is critical when designing for device performance and reliability, even for "non-mechanical" systems. However, nanoscale mechanical behavior (including failure) is inherently difficult to measure accurately, and existing modeling tools are only qualitative at best. We are developing modeling techniques that provide quantitative predictions and are validating these results experimentally.
\end{abstract}

\section{Lyle E. Levine and Anne M. Chaka (838)}

$\mathrm{M}$ echanics at the nanoscale is inherently difficult to model accurately. Finite element modeling (FEM) can effectively capture the elastic behavior of macroscopic structures but includes no accurate failure criteria since this depends upon atomic-scale behavior. Classical atomistic simulations can handle enough atoms (millions to billions) to model such events, but these potentials become inaccurate for large strains and they cannot effectively handle chemistry. Quantum-mechanicsbased simulations using density functional theory (DFT) are extremely accurate and handle the chemistry exactly, but such simulations are so CPU intensive that they can handle only a few hundred atoms. A combination of all three modeling techniques is required to accurately model device behavior at the nanoscale.

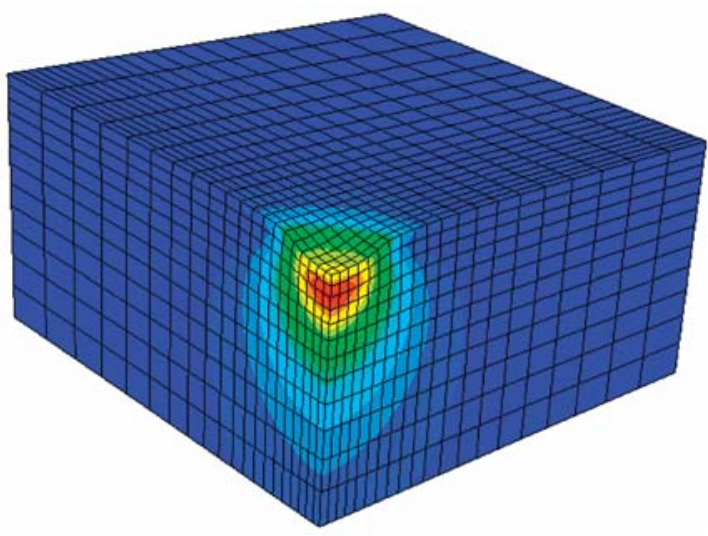

Figure 1: FEM model of a rigid $100 \mathrm{~nm}$ diameter sphere indenting an Al sample to a depth of $10 \mathrm{~nm}$.

Over the past year, we have developed techniques to handle such multiscale modeling for quasistatic applications. At the macroscale, FEM is used to simulate the elastic behavior of a nanomechanical system. Figure 1 shows an example in which an $\mathrm{Al}$ sample is being indented by a rigid $100 \mathrm{~nm}$ diameter sphere. The indenter and three of the sample quadrants have been removed to highlight the resulting Von Mises stress distribution after indenting $10 \mathrm{~nm}$. The FEM mesh is fine enough to use the predicted elastic displacement fields to generate boundary conditions and initial atom positions for an atomistic simulation using classical potentials.

The use of classical potentials in a large simulation cell allows the correct propagation of the long-range stresses to the critical regions where bond distortions are large or where chemistry effects need to be explored. In these critical regions, the techniques embed a DFT simulation. Iteratively, the critical region is relaxed using DFT, and the classical cell is relaxed using a Monte-Carlo algorithm. The first application of this hybrid technique was to determine the vacancy formation energy in aluminum as a function of distance (at a fixed angle) away from an edge dislocation. The simulation geometry is shown in Figure 2. The boundary conditions and initial atomic positions were calculated from the known elastic displacement field of an edge dislocation.

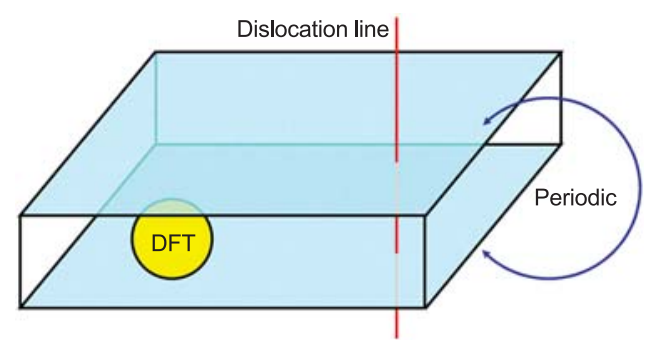

Figure 2: Diagram of a hybrid simulation for obtaining the vacancy formation energy at different positions relative to an edge dislocation. The large box represents the EAM cell in which the DFT region containing the vacancy was embedded.

After an initial dislocation nucleation event, nanoindentation progresses through the complex evolution of dislocation structures. For example, the raised lip around the indent is produced by large numbers of dislocations exiting the surface. We are working on modeling the early stages of this process using $3 \mathrm{D}$ dislocation dynamics and assuming a random distribution of dislocation sources in the sample.

Finally, connection to experimental measurements requires careful force calibration of the indenter and calibrated atomic force microscopy measurements (AFM) of the indenter tip. These calibrations are mostly complete, and the AFM data will be used to generate a FEM mesh for the simulations. Bulk single-crystal copper samples are being cut and polished using non-contact chemical methods to minimize the dislocation density.

\section{Contributors and Collaborators}

S.M. Khan, G. Levi, L. Ma, F. Tavazza (Metallurgy Division, NIST); B. Hockey, R. Machado, D. Smith, R. Wagner, D. Xiang (Ceramics Division, NIST) 


\section{Biomaterials}

New materials and devices are radically changing the treatment of injury and disease, yet it is clear that within this rapidly evolving segment of the materials industry, a basic measurement infrastructure does not exist. The Biomaterials Program develops measurement methods, standards, and fundamental scientific understanding at the interface between the materials and biological sciences. For the health care industry, we focus on dental and medical sectors that apply synthetic materials for replacement, restoration, and regeneration of damaged or diseased tissue. Three primary foci exist within this program: biocompatibility, biomaterials characterization, and materials measurements applied to biological systems.

Whether the medical issue involves implanting a hip- or knee-joint prosthesis, a synthetic bone graft, or a tissue engineering scaffold into the human body, one primary issue is biocompatibility. Using our expertise in materials science, we have developed suitable Reference Materials (RM) for investigating biocompatibility and implant suitability. Research has focused on measuring cellular response to powders and bulk materials that are candidates for implants; recently, we produced a realistic wear particle Standard Reference Material (SRM $\left.{ }^{\circledR} 2880\right)$ for bioactivity testing.

Work on quantitative methods of biomaterials characterization includes assays for adhesion, viability, proliferation, and differentiation of bone cells, 3-dimensional structural/functional imaging of tissue in-growth, and biochemical assays to quantify inflammatory responses to synthetic materials. The focus of this effort is bridging the gap between fundamental knowledge and the product development needs in industry. For example, in collaboration with the Chemical Science and Technology Laboratory, we are developing measurement methodologies and reference materials to assess interactions in complex systems of living cells with synthetic materials. The expected outcome of this work includes reference substrates that induce specific cellular responses, and engineered DNA vectors to act as fluorescent reporters of cellular responses.

Another example of our effort to bridge this gap is our collaboration with the dental industry, which is primarily composed of small manufacturers with limited R\&D capability. Collaborations with the American Dental Association Foundation (ADAF) develop improved materials and materials measurements techniques, patent and license these inventions, and, most importantly, provide a technical foundation. Research focuses on improved understanding of the synergistic interaction of the phases of polymer-based composites and the mechanisms of adhesion to dentin

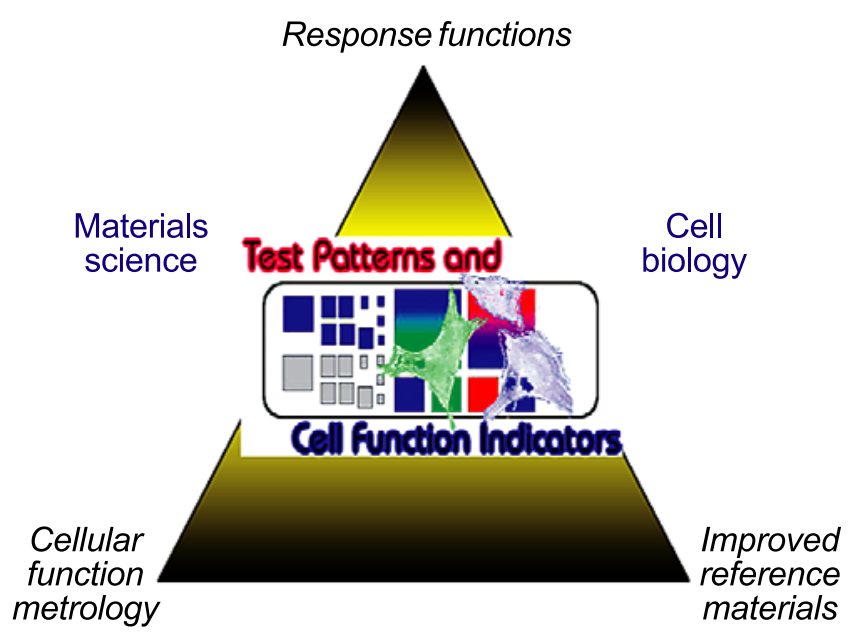

and enamel. This approach will ultimately lead to materials with improved durability, toughness, and adhesion to contiguous tooth structure. We also collaborate with the ADAF to develop metrology for the biocompatibility of synthetic bone grafts.

In this era of interdisciplinary research, we provide an added dimension. By taking a physical/mechanical approach to how cells function, respond, and remodel in interaction with synthetic materials, we provide skill sets typically absent in the biomedical community. Mechanical properties issues also arise when considering synthetic bone grafts and tissue engineering scaffolds. Complementing traditional bulk mechanical property measurements, combinatorial approaches are being developed to identify compositions and surface features that affect properties such as biocompatibility and mechanical durability.

Our mechanical property metrology extends further to biological systems that span the range from individual neurons and muscle cells to complete pulmonary arteries. This necessitates the development of unique mechanical testing platforms and application of a materials science approach to understanding integrated properties. Recently, we have developed a bioreactor capable of applying biaxial stresses and allowing monitoring of the stress and strain of a two-dimensional scaffold sheet during tissue growth.

Fundamental to the Biomaterials program is recognition of the need for an integrated systems approach. Collaborations among and between project teams are critical to progress against the ambitious goals of this program.

Contact: Eric J. Amis (Polymers Division), Lyle E. Levine (Metallurgy Division) 


\section{USAXS and USAXS Imaging of Biomaterials}

\begin{abstract}
Modeling the behavior of complex materials requires detailed knowledge of the underlying three-dimensional microstructure. Ultra-small-angle $X$-ray scattering (USAXS) imaging is a new class of synchrotron $X$-ray imaging techniques that uses USAXS as the contrast mechanism. The technique provides imaging and statistical data that cannot be obtained using any other experimental methods. It has now been applied to characterize artificial tissue scaffolds and human cartilage in $3 D$.
\end{abstract}

\section{Lyle E. Levine}

$\mathrm{U}$ SAXS imaging was developed by NIST researchers and was first demonstrated in May 2000. Advantages over existing X-ray imaging techniques are its inherently higher contrast and its USAXS-derived ability to provide quantitative data on object shapes and size distributions. USAXS imaging is applicable to a wide range of material systems including metals, ceramics, polymers, composites and biological materials. Over the past year, work has concentrated on two main areas. First, the final hardware components were installed, greatly improving the USAXS and USAXS imaging capabilities, and, second, a wide range of material systems, including biological ones, have been explored to determine where USAXS imaging can have the greatest impact.

The USAXS imaging experiments are conducted on the UNICAT sector 33 insertion-device beamline at the Argonne National Laboratory Advanced Photon Source (APS). A high-intensity, nearly-parallel, monochromatic $\mathrm{X}$-ray beam passes through entrance slits that define the size, shape and position of the beam. The angular divergence in the $\mathrm{X}$-ray beam is reduced by using multiple Bragg reflections on $<111>$ Si crystals referred to as the collimator. Within the sample, local density variations from the microstructure produce X-ray scattering at small angles. The X-rays leaving the sample are then angle-filtered using a pair of $<111>$ Si crystals referred to as the analyzer. The only X-rays from the sample that can pass through the analyzer are those scattered by the microstructure at a specific angle, where this angle can be selected by rotating the analyzer. Images are then formed by either exposing nuclear emulsion plates or using the NIST high-resolution X-ray camera system that was completed in FY 2003.

During FY 2004, the USAXS imaging instrument was declared fully operational after installation and testing of the last major hardware component, a new collimator system in which the surfaces of the two $\mathrm{Si}$ crystals were chemo-mechanically polished to minimize image aberrations. The new collimator was designed, built, and paid for by the APS and meets all of our specifications. Only minor problems were encountered during testing, and the required modifications are nearly completed.

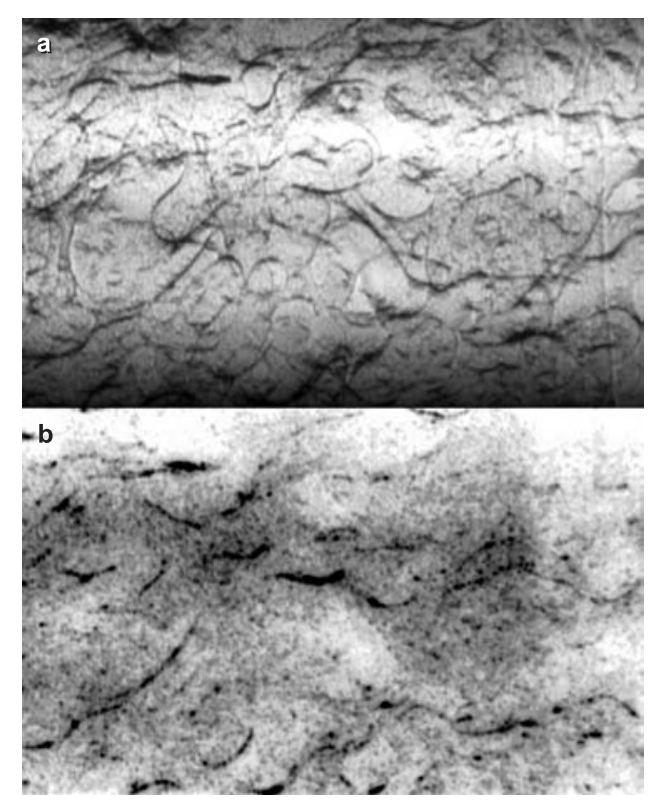

Figure 1: Identical sample volumes of an artificial tissue scaffold imaged at scattering vectors of a) $q=0 \AA^{-1}$ and b) $q=0.0005 \AA^{-1}$.

Samples studied this year include artificial tissue scaffolds composed of porous poly-caprolactone (PCL), human cartilage, several metal alloys and a composite of carbon black in poly-methylmethacrylate. Figure 1 shows images of a PCL sample with $50 \%$ porosity that was cultured with osteoblasts for 28 days. Figure 1a was taken with a $0^{\circ}$ scattering angle (radiographic) and shows the overall structure of the scaffold. Figure $1 \mathrm{~b}$, from the same sample volume, was acquired at a scattering vector of $q=0.0005 \AA^{-1}$ and shows the distribution of osteoblasts on the scaffold surfaces. Stereopairs were used to examine the cell positions in 3D. A full rotation sequence was acquired at $q=0 \AA^{-1}$, and a tomographic reconstruction produced a complete $3 \mathrm{D}$ description of the scaffold structure.

A detailed paper describing the USAXS imaging instrument along with the underlying theory was completed and accepted for publication.

\section{Contributors and Collaborators}

J. Dunkers (Polymers Division, NIST); J. Ilavsky, G. Long (Argonne National Laboratory); P. Jemian (UNICAT); C. Muehleman (Rush Medical College) 


\section{Materials for Electronics}

The U.S. electronics industry faces strong international competition in the manufacture of smaller, faster, more functional, and more reliable products. Many critical challenges facing the industry require the continual development of advanced materials and processes. The NIST Materials Science and Engineering Laboratory (MSEL) works closely with U.S. industry covering a broad spectrum of sectors including semiconductor manufacturing, device components, packaging, data storage, and assembly, as well as complementary and emerging areas such as optoelectronics and organic electronics. MSEL has a multidivisional approach, committed to addressing the most critical materials measurement and standards issues for electronic materials. Our vision is to be the key resource within the Federal Government for materials metrology development and will be realized through the following objectives:

- Develop and deliver standard measurements and data;

- Develop advanced measurement methods needed by industry to address new problems that arise with the development of new materials;

- Develop and apply in situ as well as real-time, factory floor measurements, for materials and devices having micrometer- to nanometer-scale dimensions;

- Develop combinatorial material methodologies for the rapid optimization of industrially important electronic materials;

- Provide the fundamental understanding of the divergence of thin film and nanoscale material properties from their bulk values;

- Provide the fundamental understanding of materials needed for future nanoelectronic devices, including first principles modeling of such materials.

The NIST/MSEL program consists of projects led by the Metallurgy, Polymers, Materials Reliability, and Ceramics Divisions. These projects are conducted in collaboration with partners from industrial consortia (e.g., International SEMATECH), individual companies, academia, and other government agencies. The program is strongly coupled with other microelectronics programs within the government such as the National Semiconductor Metrology Program (NSMP). Materials metrology needs are also identified through the International Technology Roadmap for Semiconductors (ITRS), the IPC Lead-free Solder Roadmap, the National Electronics Manufacturing Initiative (NEMI) Roadmap, the Optoelectronics Industry Development Association (OIDA) Roadmap, IPC (the International Packaging Consortium), and the National [Magnetic Data] Storage Industry Consortium (NSIC) Roadmap.
In each of these areas, MSEL researchers have made substantial contributions to the most pressing technical challenges facing industry, from new fabrication methods and advanced materials in the semiconductor industry, to advanced packaging materials, to magnetic data storage. Below are just a few examples of MSEL contributions over the past year.

\section{Advanced Gate Dielectrics}

To enable further device scaling, the capacitive equivalent thickness (CET) of the gate stack thickness must be $0.5 \mathrm{~nm}$ to $1.0 \mathrm{~nm}$. This is not achievable with existing $\mathrm{SiO}_{2}$ / polcrystalline $\mathrm{Si}$ gate stacks. Given the large number of possible choices for these new layers, the only feasible approach to understanding the complex materials interactions that result at the gate dielectric/ substrate and gate dielectric/metal gate electrode interfaces is through the application of combinatorial methodologies. This same methodology and apparatus are applicable to a wide variety of problems in the electronic materials field.

\section{Sub-100 nm Nanofabrication}

The continual decrease in feature size has been the driving force for advances in the semiconductor industry. Current structures have $90 \mathrm{~nm}$ dimensions with planned nodes at $65 \mathrm{~nm}$ and $35 \mathrm{~nm}$ structures. Advanced measurements of the patterning materials (photoresists), are needed to enable future large scale manufacturing of smaller devices. MSEL utilizes advanced x-ray and neutron tools to provide insight into the feasibility and optimization of these important processes.

\section{Advanced Metallization}

Electrodeposited copper is rapidly replacing aluminum for on-chip "wiring" because of its lower electrical resistivity, superior electromigration behavior, and the ability to fill fine features without the formation of seams or voids. As feature dimensions go below $100 \mathrm{~nm}$, difficulties in maintaining performance are anticipated. These issues are addressed through a combination of modeling and experimental efforts.

\section{Test Methods for Embedded Passive Devices}

Significant advantages arise if passive devices are integrated directly into the circuit board as embedded passive devices rather than discretely attached with automated assembly. New metrology methods were developed to address the needs of the electronic industry. Two test methods were completed and have received wide acceptance by industry as new methods to accelerate the development of embedded passive device technology.

Contact: Martin L. Green (Ceramics Division), Eric K. Lin (Polymers Division), Daniel Josell (Metallurgy Division) 


\section{Combinatorial/Phase Diagram Approach for Metallization to Wide-Band-Gap Semiconductors}

\begin{abstract}
The development of wide-band-gap semiconductor optoelectronic and electronic devices is hindered by poor electrical contact performance. Reliability issues for contacts include the requirements to be low-resistance, morphologically smooth and thermally stable. This project develops a strategy to improve electrical and morphological characteristics of Ohmic contacts to GaN thin films by optimizing metallization schemes using an integrated phase diagram/combinatorial approach.
\end{abstract}

\section{Albert V. Dayydov and William J. Boettinger}

$\mathrm{T}$ $\mathrm{i} / \mathrm{Al} / \mathrm{Ti} / \mathrm{Au}$ metallizations are the most commonly used electrical contact to n-type GaN-based device structures. However, the overall composition (i.e., thickness ratios) and the thermal processing of metal layers are not yet optimized. A rationale for designing the optimum metallization scheme also has not been developed. This work demonstrates that the phase diagram approach, along with a combinatorial experimentation method, is a useful tool in the design and optimization of electrical contacts to $\mathrm{GaN}$ and other wide-band-gap semiconductors.

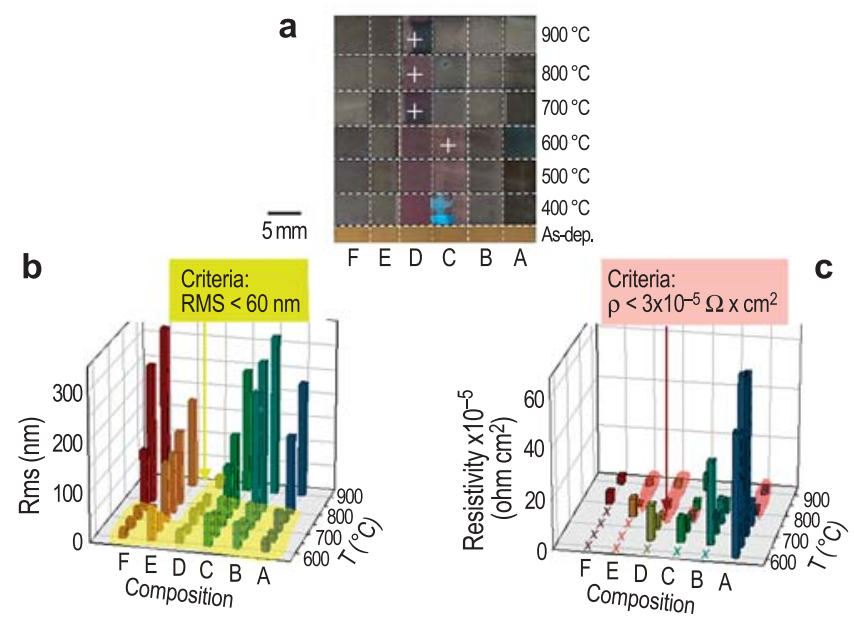

Figure 1: a) 36-element combinatorial library of annealed $\mathrm{Ti} / \mathrm{Al} / \mathrm{Ti} / \mathrm{Au}$ contacts on $n$-GaN: metal compositions change along the $x$-axis from $A$ to $F$, annealing temperature changes from $600{ }^{\circ} \mathrm{C}$ to $900^{\circ} \mathrm{C}$ along the y-axis; " $X$ "s mark the library elements with the lowest resistivity and smoothest morphology; b) map of surface roughness of the combinatorial array: yellow-shaded area represents the contacts with the smoothest ( $\mathrm{rms}<60 \mathrm{~nm}$ ) morphology; c) map of electrical resistivity of the combinatorial array: red-shaded area represents the contacts with the lowest $\left(\rho<3 \times 10^{-5} \Omega \times \mathrm{cm}^{2}\right)$ resistivity.
A combinatorial library of $\mathrm{Ti} / \mathrm{Al} / \mathrm{Ti} / \mathrm{Au}$ metallization was prepared and characterized electrically and microstructurally in order to establish optimum composition and processing parameters for the realization of high-quality Ohmic contacts. An array of metallic elements with varying $\mathrm{Ti} / \mathrm{Al} / \mathrm{Ti} / \mathrm{Au}$ thicknesses was deposited by combinatorial ion-beam sputtering (CIBS) on $\mathrm{n}-\mathrm{GaN} / \mathrm{c}$-sapphire substrate followed by rapid-thermal annealing (RTA) in the $600{ }^{\circ} \mathrm{C}$ to $900^{\circ} \mathrm{C}$ temperature range. The library design of metal compositions and RTA temperatures was guided using the Al-Au-Ti phase diagram so that various $\mathrm{Ti} / \mathrm{Al} / \mathrm{Ti} / \mathrm{Au}$ contact compositions represented different regions on the phase diagram.

Four-probe transmission-line-method and Hall electrical measurements were used to assess contact resistivity and $\mathrm{GaN}$ sheet resistance. X-ray diffraction, white-light interferometric microscopy, atomic-force microscopy, field-emission scanning-electron-microscopy, and transmission-electron-microscopy were used to evaluate the microstructure and morphology of contacts and the metal/GaN interfaces. The most Au-rich/Ti-poor metallization (E in Figure 1a), $\operatorname{Ti}(20 \mathrm{~nm}) / \mathrm{Al}(85 \mathrm{~nm}) /$ $\mathrm{Ti}(15 \mathrm{~nm}) / \mathrm{Au}(150 \mathrm{~nm})$ with an RTA of $800^{\circ} \mathrm{C} / 30 \mathrm{~s}$ in argon, produced the lowest $\left(\rho=1.3 \times 10^{-5} \Omega \times \mathrm{cm}^{2}\right)$ contact resistivity with reasonable $(\mathrm{rms}<150 \mathrm{~nm})$ surface morphology. However, the most Al-rich metallization (D in Figure 1a), $\operatorname{Ti}(20 \mathrm{~nm}) / \operatorname{Al}(170 \mathrm{~nm}) / \operatorname{Ti}(5 \mathrm{~nm}) /$ $\mathrm{Au}(50 \mathrm{~nm})$ with RTA of $750{ }^{\circ} \mathrm{C} / 30 \mathrm{~s}$ in argon, produced superior surface morphology $(\mathrm{rms}=20 \mathrm{~nm})$ and comparable resistivity of $\rho=2.2 \times 10^{-5} \Omega \times \mathrm{cm}^{2}$. It is suggested that scheme (E) with the Au-rich contact can be used for operating in oxidizing environments, where thick Au top layer will serve as a protective cap layer, while scheme (D) can be suitable for applications where contact surface roughness is critical. According to XRD results, the superior surface morphology of contacts in the D-series correlates with the absence of phase transformations in the $600{ }^{\circ} \mathrm{C}$ to $900{ }^{\circ} \mathrm{C}$ temperature interval after the initial formation of $\mathrm{Al}_{2} \mathrm{Au}$ and $\mathrm{Al}_{3} \mathrm{Ti}$ phases in metal films.

The advanced metallizations developed in the project will be validated in industrial high-electron mobility transistors (HEMTs) at Northrop-Grumman Corporation.

\section{Contributors and Collaborators}

L.A. Bendersky, D. Josell, U.R. Kattner, A.J. Shapiro (Metallurgy Division, NIST); J.E. Blendell, R.S. Gates, P.K. Schenck, M.D. Vaudin (Ceramics Division, NIST); T. Zheleva (Army Research Laboratory); H.G. Henry (Northrop-Grumman); Q.Z. Xue (Intematix Corp.); A. Motayed (Howard University); I. Takeuchi (Univ. of Maryland) 


\section{Lead-Free Surface Finishes: Sn Whisker Growth}

As the microelectronics industry moves towards $\mathrm{Pb}$-free assemblies, reliability has become a major concern. Pb-free coatings of nearly pure tin, used as a protective layer to maintain solderability on Cu leadframes and connectors, tend to grow "whiskers" which can cause shorts across component leads. Copper additions to the Sn protective layer have been considered by industry since the $\mathrm{Sn}-\mathrm{Cu}-\mathrm{Ag}$ is likely to be the Pb-free bulk solder of choice for industrial applications. However, measurements made at NIST indicate that the addition of $\mathrm{Cu}$ tends to promote whisker growth. Our goal is to better understand how copper and other solutes impact the growth of whiskers on electrodeposited Sn.

\section{Gery R. Stafford, William J. Boettinger, and Kil-Won Moon}

$\mathrm{W}$ hiskers are generally believed to grow to relieve residual stress in tin and other coatings. However, the origin of this stress has not been definitively determined. The addition of certain solutes, such as $\mathrm{Bi}$ and $\mathrm{Pb}$, are known to retard whisker growth in electrodeposited tin coatings, while solutes, such as $\mathrm{Zn}$ are known to promote growth. Previous work at NIST has shown that low levels of $\mathrm{Pb}$ reduce the residual compressive stress in $\mathrm{Sn}-\mathrm{Pb}$ electrodeposits, while the addition of $\mathrm{Cu}$ increases the residual compressive stress. Current research focuses on the influence of solute type and concentration on residual film stress, stress relaxation, and whisker formation.

Metallographic analysis of $\mathrm{Sn}-\mathrm{Cu}$ electrodeposits indicates that the $\mathrm{Cu}$ is present as the $\mathrm{Cu}_{6} \mathrm{Sn}_{5}$ intermetallic compound (IMC) which is observed both in the grains and along the grain boundaries. It is unlikely that significant amounts of this complex IMC are formed directly during deposition. Rather, we believe that the as-deposited alloy is a supersaturated solid solution of $\mathrm{Cu}$ in $\mathrm{Sn}$ that transforms to an equilibrium mixture of $\mathrm{Sn}$ and $\mathrm{Cu}_{6} \mathrm{Sn}_{5}$ either during or soon after deposition.

In solid solution, $\mathrm{Cu}$ is presumed to occupy interstitial sites within relatively large square channels that exist in the body-centered tetragonal (bct) Sn structure. This gives rise to the anomalously fast diffusion of $\mathrm{Cu}$ in the c-direction of the Sn lattice. In this configuration, it is assumed that the $\mathrm{Cu}$ does not alter the lattice volume of the $\mathrm{Sn}$. It is further presumed that as $\mathrm{Cu}_{6} \mathrm{Sn}_{5}$ precipitates within the deposit, the $\mathrm{Cu}$ exits the interstitial sites in the Sn phase and occupies volume more typical of its atomic size; thus, the volume of the entire deposit increases.
We estimate the volumetric strain for $\mathrm{Cu}_{6} \mathrm{Sn}_{5}$ precipitation from a $1 \% \mathrm{Cu}$ solid solution to be about +0.004 . The resultant stress generated in the electrodeposit as a result of the IMC precipitation is then on the order of $-95 \mathrm{MPa}$ (compressive). This, of course, assumes that $\mathrm{Cu}_{6} \mathrm{Sn}_{5}$ forms from the solid solution after the deposit has been formed.

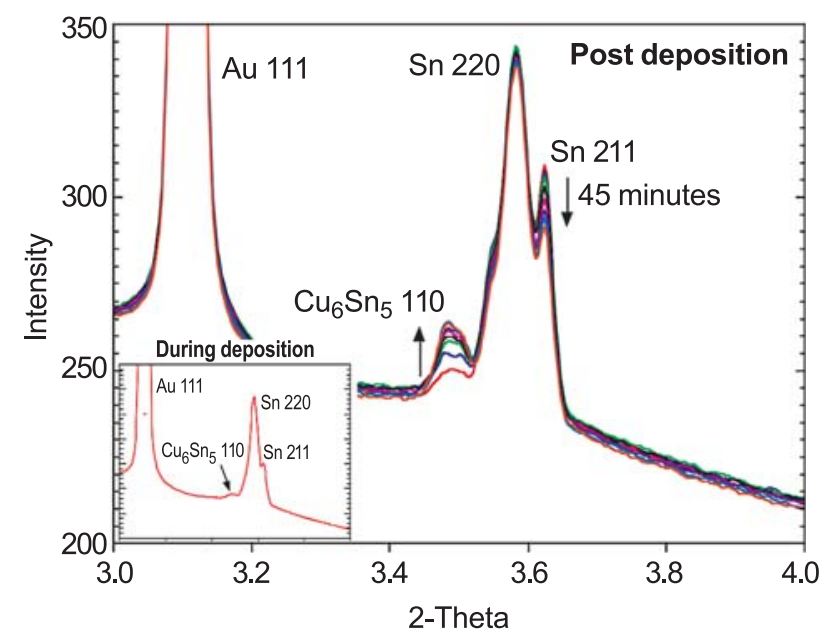

Figure 1: In situ $x$-ray diffraction $(\lambda=0.127 \AA)$ showing growth of the $\mathrm{Cu}_{6} \mathrm{Sn}_{5} 110$ reflection in the 45 minutes following deposition of bright $\mathrm{Sn}$-Cu alloy from commercial methanesulfonate electrolyte.

Figure 1 shows a series of x-ray diffraction patterns that were taken at the Advanced Photon Source, using synchrotron radiation, during and after the deposition of a $\mathrm{Sn}-\mathrm{Cu}$ alloy onto a tungsten substrate. The inset pattern was taken during the 2-minute deposition while the remaining patterns were taken 3 minutes apart after deposition was complete. The data show that although a small amount of $\mathrm{Cu}_{6} \mathrm{Sn}_{5}$ is present when deposition is stopped (inset), the bulk of the IMC forms in the 45 minutes following deposition. Although the $\mathrm{Sn}$ reflections decrease as the IMC forms, the peaks do not change position indicating that the lattice volume of the $\mathrm{Sn}$ is constant. This supports the assumption that interstitial $\mathrm{Cu}$ does not alter the lattice volume of bct $\mathrm{Sn}$. These results indicate that IMC formation occurs after deposition is complete and is a likely cause of the higher compressive stress observed in $\mathrm{Sn}-\mathrm{Cu}$ alloy deposits.

\section{Contributors and Collaborators}

J.E. Guyer, C.E. Johnson, M.E. Williams (Metallurgy Division, NIST); M.D. Vaudin (Ceramics Division, NIST); D.R. Robinson, D. Wermeille (Advanced Photon Source, ANL) 


\section{Lead-Free Solders and Solderability}

Solders and solderability are increasingly tenuous links in the assembly of microelectronics as a consequence of ever-shrinking chip and package dimensions and the international movement toward environmentally friendly lead-free solders. In collaboration with the NEMI Pb-Free Assembly Project, we are providing the microelectronics industry with measurement tools, data, and analyses that address national needs in the implementation of lead-free solders.

\section{Ursula Kattner and Carol Handwerker}

Since 1999, NIST has served a major role in National Electronics Manufacturing Initiative (NEMI) projects to assist the microelectronics industry implement $\mathrm{Pb}$-free solders. NEMI is an industry-led consortium of approximately 65 electronics manufacturers, suppliers and related organizations brought together to facilitate leadership of the North American electronics manufacturing supply chain. This move toward $\mathrm{Pb}$-free solders is a direct result of the European Union ban, starting in 2006, on Pb-containing solders in electronic products.

NEMI and NIST have worked together to respond to the identified needs by:

1) Identifying and providing the most important lead-free solder data for the microelectronics community (including the definitive database on thermodynamic properties of $\mathrm{Pb}$-free alloys), providing an analysis of the mechanical behavior of $\mathrm{Sn}-\mathrm{Ag}-\mathrm{Cu}$ alloys, and disseminating such data on the NIST website (see "Delivery of Thermodynamics and Kinetic Data" in this volume).

2) Developing and widely disseminating a Recommended Practice Guide on Test Procedures for Developing Solder Data.

3) Providing a list of literature references on alloys, processing, reliability, environmental issues, and components for the implementation of lead-free solders.

4) Completing the microstructure-based failure analysis on all thermally cycled assemblies as part of the NEMI project's full-scale reliability trials.

5) Providing chapters on the materials science of $\mathrm{Pb}$-free solder alloys for inclusion in four separate books on $\mathrm{Pb}$-free solder implementation.

Although the NEMI Pb-Free Assembly Project ended in 2003, in 2004 we continued to serve as an information resource to the microelectronics industry on issues related to the effects of alloy composition, reflow temperature and furnace profiles for best melting and solidification behavior of solders when circuit boards are assembled, and to the effects of $\mathrm{Pb}$ contamination on alloy melting and assembly reliability. This activity culminated in a Circuits Assembly article by Alan Rae, VP of Technology for Cookson Electronics, and Carol Handwerker of NIST on the range of acceptable alloy compositions in the $\mathrm{Sn}-\mathrm{Ag}-\mathrm{Cu}$ system. The following excerpt gives the NEMI position, and effectively the U.S. position, on the acceptable composition ranges for the new international standard $\mathrm{Pb}$-free alloy and allows both the U.S. and the Japanese preferred alloy compositions based on analyses of NIST thermodynamic data:
Although the composition of some of the alloys being commercialized varies slightly from the NEMI composition, the NEMI alloy is representative of the acceptable range of lead-free solders. Tin-silver- copper formulations with silver content between 3.0 [mass] $\%$ and $4.1 \%$ and copper between $0.5 \%$ and $1.0 \%$ are virtually indistinguishable in terms of melting point and process features. The NEMI alloy provides a model system for industry that is well characterized, and several NEMI members currently are using the alloy in production. The focus on a single lead-free alloy has helped to accelerate industry convergence on standard solder formulations, manufacturing processes and, ultimately, the timely and cost-effective conversion to lead-free assembly.

"The NIST group performed a significant service to industry by being the focal point for development of a reliable technology base to support the choice of a new lead-free alloy. They also led the way for further work on issues such as tin whiskers, which emerged from the initial lead-free work. NIST provided not only a strong technical basis but, by verifying reliability and comparing alternatives, enabled industry to choose an alloy based on extensive investigation."

\section{Dr. Robert C. Pfahl, Jr., Director International and Environmental R\&D, Motorola, (retired), and Vice President Operations, NEMI, (current)}

\section{Contributors and Collaborators}

W.J. Boettinger, K.W. Moon, T. Siewert, D. McCowan, L.C. Smith, S.W. Claggett, M.E. Williams (NIST); J.P. Clech (EPSI, Inc.); J. Bath (Solectron); R. Gedney (NEMI); E. Bradley (Motorola); J.E. Sohn (NEMI, formerly Lucent); A. Rau (Cookson Electronics); E. Benedetto (HP); R. Charbonneau (StorageTek) 


\section{Electrical Properties of On-Chip Interconnections}

Copper wiring in on-chip electrical conductors has reached dimensions so small that electrical resistivity is no longer constant. The higher resistivity induces greater power dissipation, aggravating chip cooling problems. We have quantified the microstructural factors behind this increase for silver wires, where silver has the highest electrical conductivity of any metal.

\section{Daniel Josell and Thomas P. Moffat}

\begin{abstract}
A dimensions of transistors in integrated circuits shrink, the dimensions of the metal wires connecting them shrink as well. Dimensions of the wires, and internal grains, are now at approximately $100 \mathrm{~nm}$, similar to the intrinsic mean-free-path lengths of the conduction electrons. Scattering from wire surfaces and grain boundaries is significantly reducing electrical conductivity in such small conductors.
\end{abstract}

Upcoming dimensional requirements, as specified in the International Technology Roadmap for Semiconductors, require determination of the origin and magnitude of the resistivity increase in order to direct research that might mitigate these size effects.

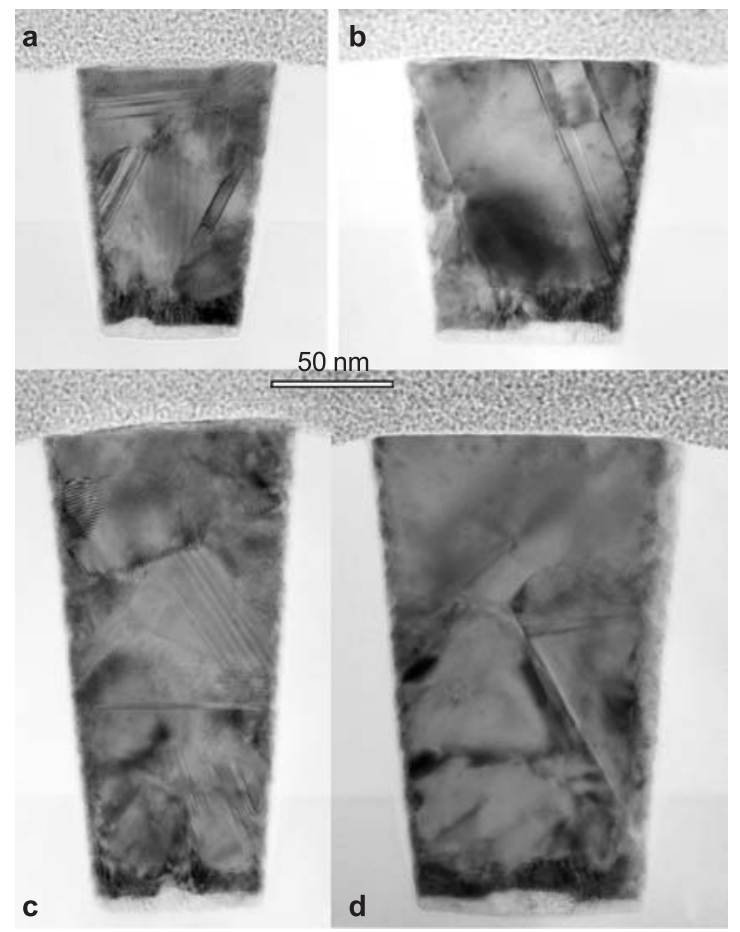

Figure 1: Transmission electron microscope images of silver wires cross-sectioned after electrical measurement. The higher aspect ratio (height/width) wires contain multiple grains while the lower aspect ratio wires contain only twin boundaries.
To address these issues, we have measured size-dependent resistivity of silver wires $100 \mathrm{~nm}$ to $300 \mathrm{~nm}$ tall for widths ranging from approximately $50 \mathrm{~nm}$ to $840 \mathrm{~nm}$, produced by electrodeposition on patterned wafers followed by a combined chemical mechanical planarization and oblique ion-polishing process. Silver was selected because of its high electrical conductivity and our recent development of a bottom-up "superfill” process for creating void-free and seam-free wires (Figure 1).

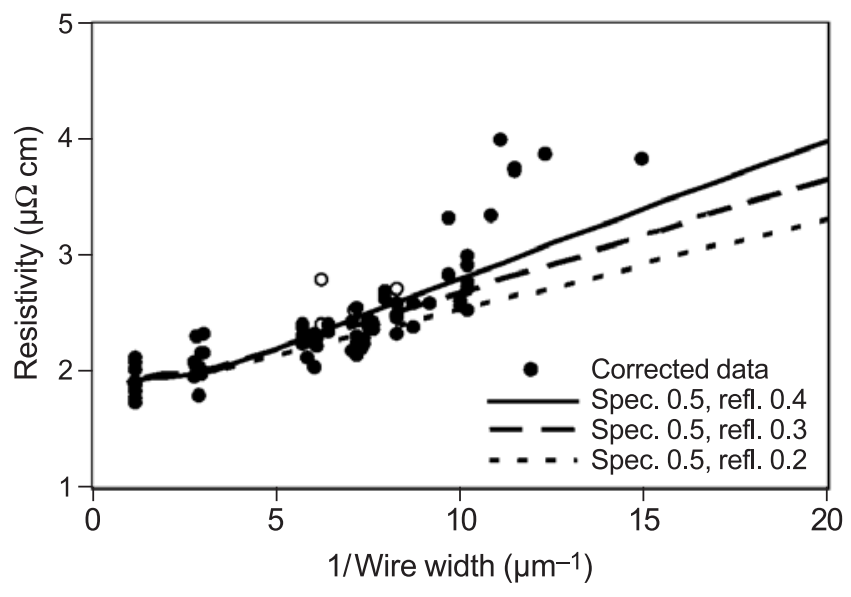

Figure 2: Electrical properties of $300 \mathrm{~nm}$ tall silver wires as a function of the wire width. Curves are the result of calculations including intrinsic, surface and grain boundary scattering of electrons. The sharp increase at $\approx 100 \mathrm{~nm}$ is associated with defects.

To interpret the resistivity data, the Fuchs-Sondheimer formalism (diffuse scattering of electrons on the wire surfaces) was extended to permit a nonzero specular component for surface scattering. The analysis used an existing formalism to account for grain boundary scattering. The resulting equations permit quantitative comparison to experiment (Figure 2).

Significantly, the analysis shows that surface scattering alone is not capable of explaining the observed behavior. Grain boundary scattering is found to contribute a similar, or larger, amount to the overall increase. As a result, the potential payoff of increasing the grain size to decrease grain boundary scattering is recognized to be at least as large as that which might be obtained by smoothing surfaces to increase specularity.

\section{Contributors and Collaborators}

G. McFadden (Mathematical and Computational Sciences Division, NIST); R.R. Keller, Y.-W. Cheng (Materials Reliability Division, NIST); C. Witt (CooksonEnthone); C. Burkhard, Y. Li (Clarkson University) 


\section{Nanomagnetodynamics}

In order to pursue the rapid development path set out for hard drives and magnetic memory, industry needs the ability to measure and control magnetization on nanometer length scales and nanosecond time scales. This project focuses on the metrology of dynamics and damping in magnetic thin films, especially on the effects of nano-scale dimensions and defects in ferromagnetic resonance measurements.

\section{Robert D. McMichael}

$\mathrm{W}$ e are developing ferromagnetic resonance (FMR) techniques to measure the static and dynamic properties of thin films and patterned arrays of technologically important ferromagnetic metals and their interfaces with normal metals. The primary results include measurements of surface anisotropy, which becomes increasingly important for nanoscale damping, which governs behavior on the sub-nanosecond time scale, and assessment of the magnetic homogeneity of thin films or patterned arrays of magnetic bits. These results are communicated to the magnetic data storage industry through conference presentations, journal articles and site visits.

For nanostructured materials, surface effects and interfacial effects are important. For example, micromagnetic calculations show that switching behavior of magnetic memory cells will be affected by surface anisotropy on the edges of the lithographically patterned elements, an effect that has not been appreciated until recently. Measuring the anisotropy of films of varying thickness with ferromagnetic resonance enables the determination of surface anisotropy values for Permalloy that will be used in the micromagnetic design of MRAM cells.

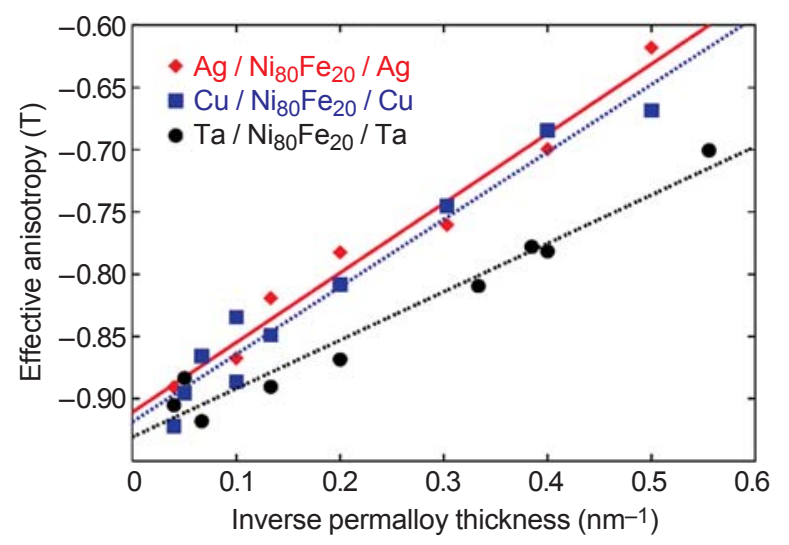

Figure 1: Surface anisotropy measurements for Permalloy films with different normal metal over/underlayers. The slopes of the fit lines correspond to surface anisotropy.
A continuing thrust of this project has been the development of models that describe broadening of the FMR linewidth by defects. These models, which are specifically designed for thin films, account for both inhomogeneity and magnetic interactions. Our earlier models were restricted to "local" defects such as anisotropy. One important application of these models is as a method for measurement of anisotropy variations and axis alignment in perpendicular recording media. This year, we have capped off the model development with a model for the non-local effects of film roughness.

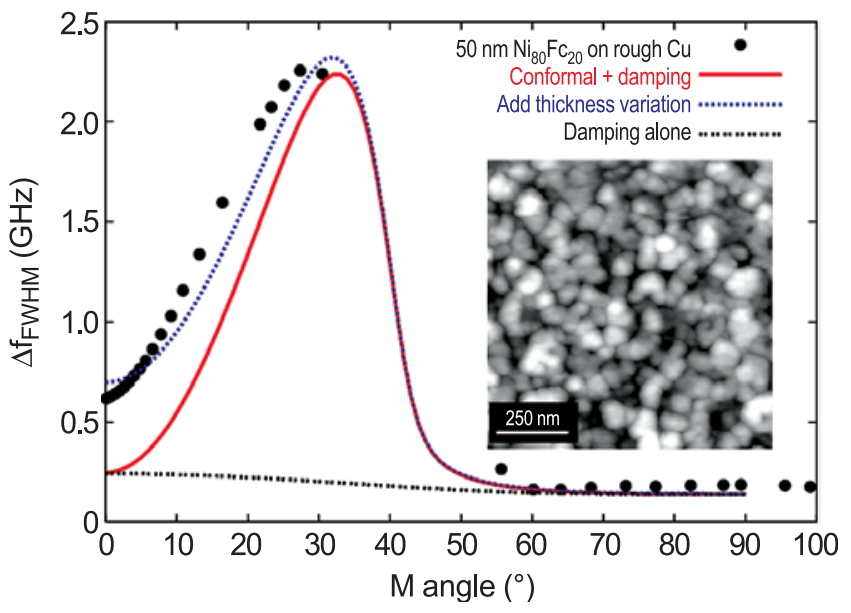

Figure 2: FMR line broadening measured in a Permalloy film and calculated almost entirely from the microstructure of the underlayer shown in the inset.

Testing of the roughness-linewidth model involved making variable roughness substrates, characterizing the roughness by atomic force microscopy (AFM), and measuring nearly conformal Permalloy overlayers in FMR. Using only the AFM microstructural data and the known properties of Permalloy, and assuming conformal roughness, the model predictions agreed extremely well with the measurements. Allowing one free parameter describing thickness variations, the agreement with the experiment is nearly perfect. Validation of this model is the first example of linewidth modeling based entirely on the measured microstructure.

\section{Contributors and Collaborators}

J.O. Rantschler, B.B. Maranville, J. Mallett, T. Moffat, W.F. Egelhoff, Jr., A.P. Chen (Metallurgy Division, NIST); J.A. Borchers, S.K. Satija (NIST Center for Neutron Research); W. Bailey (Columbia U.); A. Arrott (Virginia State University) 


\section{Electrodeposited $\mathrm{Pt}_{1-\mathrm{x}}(\mathrm{Fe}, \mathrm{Co}, \mathrm{Ni})_{x}$ Alloys}

Platinum-iron group alloys have at least two important potential applications: as a medium for magnetic recording and as $C O$ tolerant catalysts in fuel cells. Alloy formation by electrodeposition presents a convenient and inexpensive alternative to conventional vacuum methods. A simple thermodynamic model has been used to account for the observed dependence of alloy composition on the deposition potential.

\section{Jonathan J. Mallett, William F. Egelhoff, Jr., and Thomas P. Moffat}

$\mathrm{P}$ erhaps the most exciting application of iron-group $\mathrm{Pt}$ alloys involves their high magnetic coercivity and anisotropy in the $\mathrm{L} 1_{0}$ phase. This makes them ideal candidates for future magnetic storage media, which will require high coercivity material to sustain the reductions in bit size needed to increase storage density. Controlled alloy composition and microstructure are essential for achieving the $\mathrm{L}_{0}$ phase, which requires close to a 50:50 composition.

Iron-group Pt alloys may also find application as poison-resistant hydrogen fuel cell catalysts. A primary concern is the lifetime and efficiency of the catalyst used to oxidize hydrogen gas in the cell. Recent research suggests that replacing the traditional Pt catalyst with a Pt alloy containing up to $15 \%$ of an Fe-group element can dramatically improve the resistance of the catalyst to $\mathrm{CO}$ poisoning. An inexpensive, reliable process for producing such alloys is, therefore, likely to be of considerable utility to fuel cell development.

An electrodeposition method has been developed that allows smooth ( $<5 \mathrm{~nm}$ RMS roughness) alloy films to be deposited to a thickness of between $20 \mathrm{~nm}$

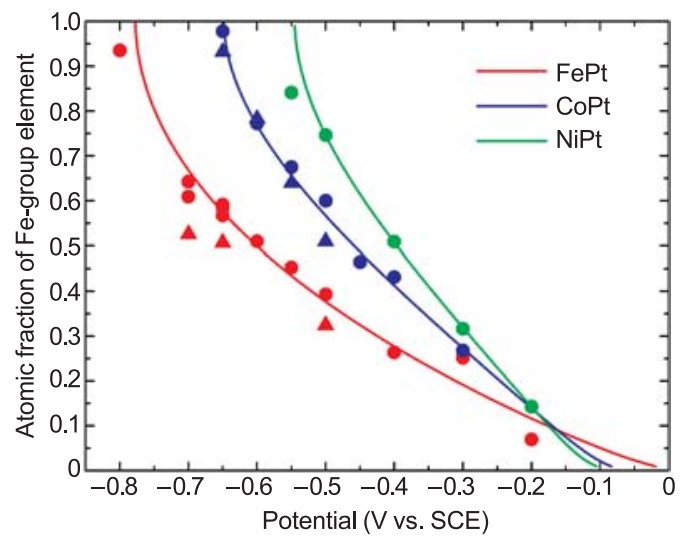

Figure 1: The theoretical (curves) and experimental (data points) dependence of composition on potential. and $3000 \mathrm{~nm}$. Deposition takes place from a bath containing $3 \mathrm{mM} \mathrm{PtCl}_{4}$ and $0.1 \mathrm{M}$ of the reactive metal ions $\left(\mathrm{Fe}^{2+}, \mathrm{Ni}^{2+}\right.$, or $\left.\mathrm{Co}^{2+}\right)$. The deposition potentials were chosen such that deposition of the reactive metal in its bulk form would have been energetically impossible. Platinum on the other hand readily deposits at all of the chosen potentials. The incorporation of the reactive metal occurs due to the highly negative enthalpy of mixing of the alloy. This reduces the activity of the reactive element in the alloy and allows its deposition to occur at more positive potentials than would be possible for the pure element. The reactive ions are in much higher concentration than the noble complexed platinum ions, which allows the former to be reduced in a kinetically facile way that enables equilibrium alloy compositions to be achieved. Under these conditions, the alloy composition and morphology was found to remain remarkably invariant to significant perturbations in constituent concentrations. The strong dependence of composition on potential is shown in Figure 1 for three alloys.

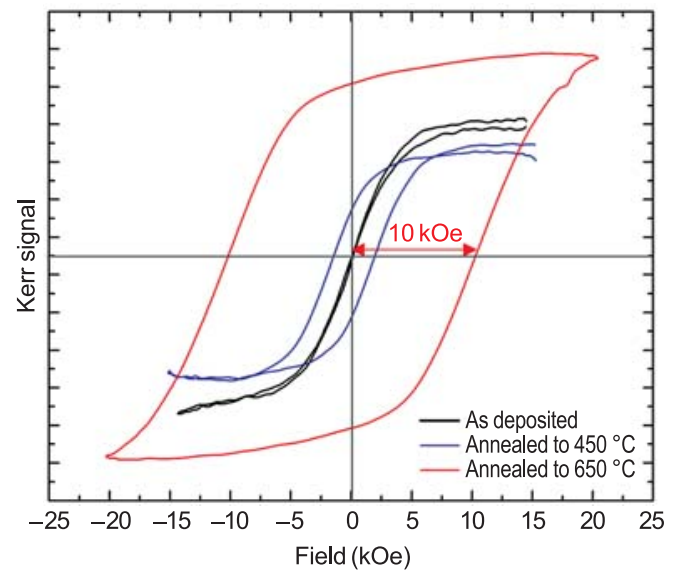

Figure 2: The increase in coercivity on annealing.

Figure 2 shows the effect of annealing an FePt film grown on a $\mathrm{Cu}(001)$ substrate. The transformation to the $\mathrm{L1}_{0}$ phase results in a dramatic increase in magnetic coercivity. The 10 kOe coercivity produced by the $650{ }^{\circ} \mathrm{C}$ anneal is suitable for next-generation magnetic media.

Current activities are focused on extending the investigation to ternary alloys and expanding the available deposited structures to include nanoparticles (useful as catalysts) and template-formed nanostructures (for future magnetic recording media).

\section{Contributors and Collaborators}

E.B. Svedberg (Seagate Technology) 


\section{Novel Magnetic Materials for Sensors and Ultra-High Density Data Storage}

\begin{abstract}
Magnetic sensors play a central role in many important technologies ranging from health care to homeland security. A common need among these technologies is greater sensitivity and smaller size. In ultra-high density data storage, one of the most pressing needs is for nano-structured media that store data at ever-increasing densities. Improved methods for the magnetic isolation of grains in ultra-thin films are a key need; we have initiated research programs in both areas.
\end{abstract}

William F. Egelhoff, Jr.

$\mathrm{N}$ IST's Magnetic Engineering Research Facility (MERF) is one of the most versatile facilities in the world for the fabrication and analysis of novel magnetic thin films. Two new areas of research, magnetic sensors and magnetic media, illustrate this versatility. The common link is that both require novel magnetic thin films.

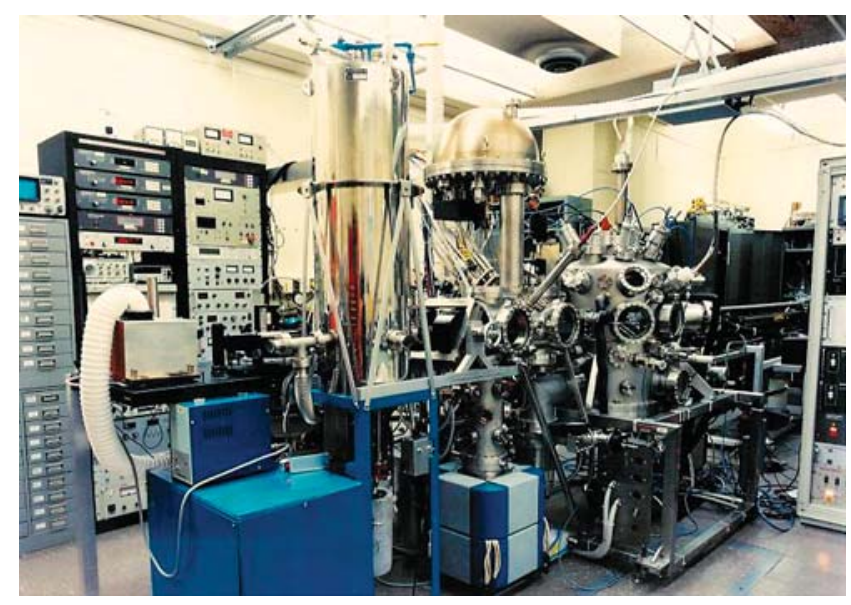

Figure 1: The Magnetic Engineering Research Facility (MERF).

It appears that ultra-sensitive magnetic sensors will require the use of thin films of ultra-soft magnetic alloys. These alloys often have a very complex composition such as $\mathrm{Fe}_{73.5} \mathrm{Si}_{13.5} \mathrm{~B}_{9} \mathrm{Nb}_{3} \mathrm{Cu}_{1}$ and require a very specific annealing process. These materials are well-known in bulk form but have never been produced in thin-film form. The project goal is to carry out the metrology needed to produce these materials as ultra-thin films.

We are presently modifying the MERF to add the capability of ion-beam sputter deposition (IBSD). IBSD, which is based on sputtering a target of the desired composition, is the only reliable method for maintaining the correct composition for complex ultra-thin films. MERF will soon become the only deposition system in the world equipped for ultra-thin film deposition by IBSD, $\mathrm{AC}$ and DC magnetron sputtering, and molecular beam epitaxy. Such versatility will allow MERF to continue to play a leading role in magnetic thin-film research.

In the area of novel magnetic media we have been collaborating closely with Seagate. Seagate is the world's leading manufacturer of hard-disk drives, and for the past two years, they have been sending a Ph.D. physicist from their research labs to work with us, first on ballistic magnetoresistance, and now on novel magnetic media.

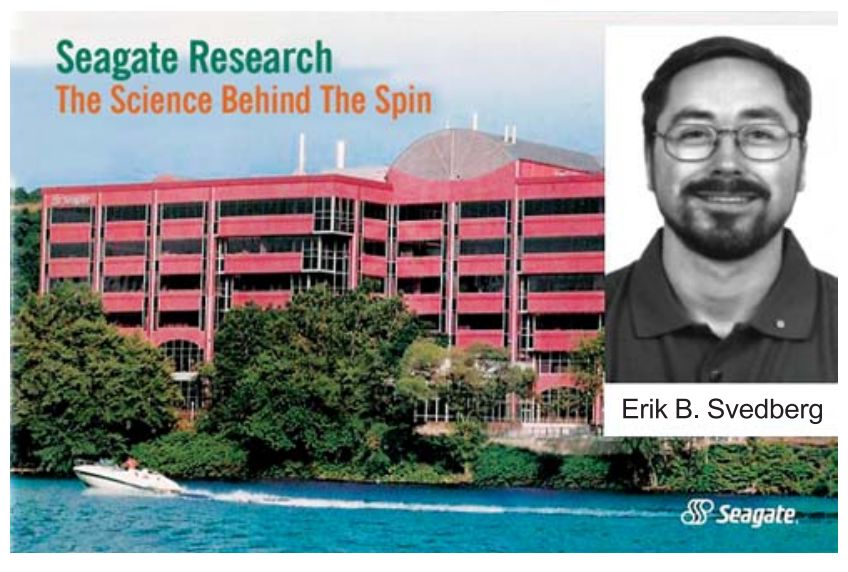

Figure 2: Close collaborations with Seagate have contributed to the success of this project.

Recent work determined a novel method for magnetically decoupling grains in CoPd media. CoPd multilayers are one of the leading candidates for the next generation of magnetic media. We have found that if a Au film is deposited on top of the CoPd and anneal in air, two unexpected phenomena occur as a result of rapid diffusion of atoms along grain boundaries. One is that Co atoms diffuse to the surface, react with oxygen, and remain at the surface. The other is that $\mathrm{Au}$ atoms diffuse into the grain boundaries replacing Co. The net effect is that the grain boundaries are demagnetized, and each grain can magnetically switch independently of its neighbors. This method provides the best magnetic isolation of grains yet found.

\section{Contributors and Collaborators}

E.B. Svedberg, D. Weller (Seagate); R.D. McMichael, T.P. Moffat, J. Mallett (Metallurgy Division, NIST); D.P. Pappas (Electromagnetics Division, NIST) 


\section{Discovery of Spin Density Waves in a Ferromagnet: Fe-Al}

Magnetism is endemic to our society. Ferromagnets are the basis for information storage in computers and recording devices; they are found in all motors, transformers, and generators; and they are used in a host of other products like credit cards, cellular telephones, radios, and televisions. However, the magnetic characteristics of the ferromagnet used in each of these applications varies with the product. Consequently, industry needs to know the interconnection between material characteristics and magnetic properties, and how to control them. A critical element in such an understanding is knowledge of the fundamentals of spin interaction, and how to measure it. NIST provides the metrology needed by industry to do this.

\section{Robert D. Shull and Jeffrey W. Lynn (NCNR)}

Tn 1962, a new type of interaction between magnetic 1 spins was predicted - a band structure effect caused by an instability in the conduction electron gas near the Fermi surface. The instability creates a new low-energy state in which the magnetic spin orientations form a standing wave, called a spin density wave (SDW). Similarly, a wave of charge, called a charge density
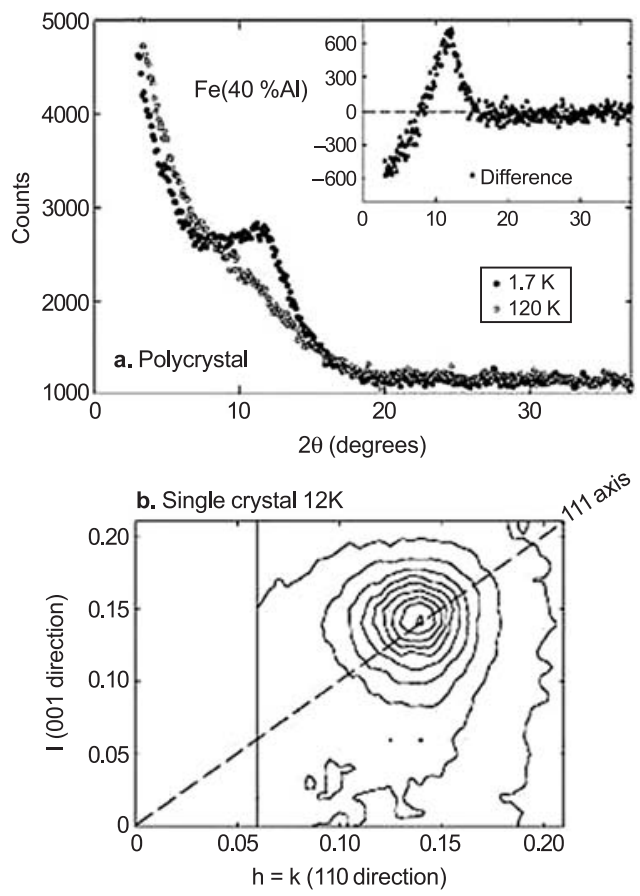

Figure 1: Neutron scattering intensity for a) polycrystalline $\mathrm{Fe}_{60} \mathrm{Al}_{40}$ at $120 \mathrm{~K}$ and $1.7 \mathrm{~K}$ with their difference in the inset and b) single crystal $\mathrm{Fe}_{60} \mathrm{Al}_{40}$ at $12 \mathrm{~K}$ shown as contours about the $(1 / 7,1 / 7,1 / 7)$ peak. wave (CDW), was also predicted. Charge density waves have since been found experimentally, and the SDW concept has been successfully used to explain the unusual incommensurate antiferromagnetism in $\mathrm{Cr}$. However, evidence for SDWs in a ferromagnet has proven elusive.

Using neutron diffraction measurements at NIST, we recently discovered a SDW in a single crystal of $\mathrm{Fe}_{60} \mathrm{Al}_{40}$, a material with ferromagnetically interacting spins. This was shown (Figure 1) by the presence of scattering intensity at locations where there was no atomic reciprocal lattice vector (i.e., at places where there would normally be no coherent atomic scattering). In fact, the data showed there are several SDWs in the material with wave vectors near (110) directions (shown in Figure 2). No such waves were found along either (100) or (111) directions.

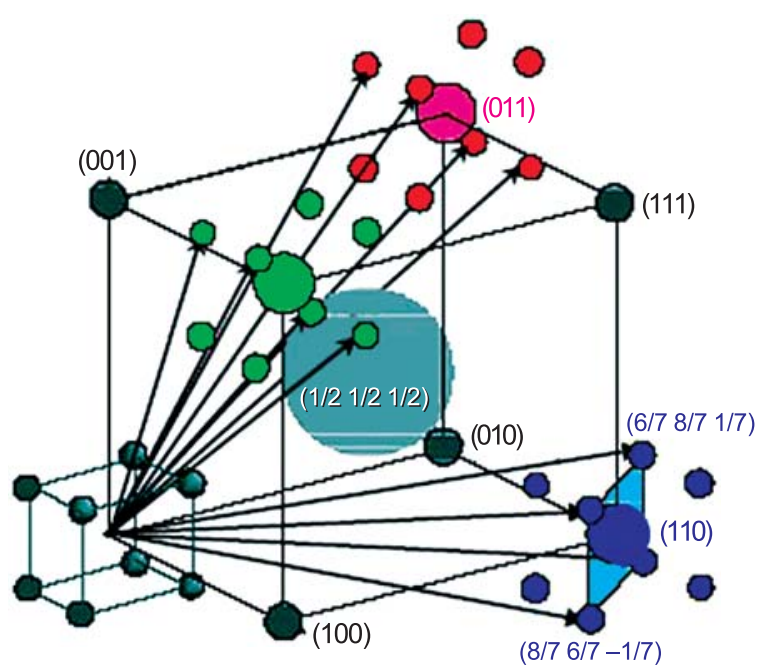

Figure 2: The reciprocal lattice spots in $\mathrm{Fe}_{60} \mathrm{Al}_{40}$, showing the $S D W$ wave vectors (black arrows) (Physical Review Letters 91, 217201-1 (2004).

The presence of SDWs shows the exchange interactions between magnetic spins in Fe-Al is a band structure (i.e., non-local) effect and explains the large magnetism in these materials containing a large content of "non-magnetic" atoms. This discovery should be as important to the understanding of magnetism in metals as the SDWs in $\mathrm{Cr}$. In $\mathrm{Cr}$, the $\mathrm{SDW}$ wave vector, close to a (100) direction, makes moments of nearest neighbors in the bcc structure almost oppositely aligned.

\section{Contributors and Collaborators}

A.S. Arrott (Virginia State University); D.R. Noakes (Virginia State University); M.G. Belk (Virginia State University); S.C. Deevi (Phillip Morris Co.); D. Wu (Dartmouth College) 


\section{Safety and Reliability}

While various structural failures have captured national attention over the years, the events of September 11, 2001 generated a greatly increased awareness of vulnerabilities in our national infrastructure. The extent of these vulnerabilities depends to a large degree on the performance of materials in situations outside of the original design considerations. It is now recognized that a critical and urgent national need exists to establish the behavior of materials under such extreme loadings, and to disseminate guidance and tools to assess and reduce future vulnerabilities.

The goal of providing a technical basis for cost-effective changes to national practices and standards, coupled with a need for an integrated effort drawing on capabilities and expertise of a broad collaborative team, has lead to the development of the Safety and Reliability Program within MSEL. This program draws on the expertise of several divisions in MSEL and across NIST.

Project selection is guided by an identification and assessment of the particular vulnerabilities within our materials-based infrastructure, and focusing on those issues which would benefit strongly by improved measurements, standards, and materials data. Ultimately, we intend to moderate the effects of acts of terrorism, natural disasters, or other emergencies, all through improved use of materials.

Our vision is to be the key resource within the Federal Government for materials metrology development as realized through the following objectives:

- Identify and address vulnerabilities and needed improvements in U.S. infrastructure;

- Develop and deliver standard measurements and data;

- Develop advanced measurement methods needed by industry to address new problems that arise with the development of new materials;

- Support other agency needs for materials expertise.

This program responds both to customer requests (primarily other government agencies) and to the Department of Commerce 2005 Strategic Goal of "providing the information and framework to enable the economy to operate efficiently and equitably." For example, engineering design can produce safe and reliable structures only when the property data for the materials is available and accurate. Equally important, manufacturers and their suppliers need to agree on how material properties should be measured.

The Safety and Reliability Program works toward solutions to measurement problems on scales ranging

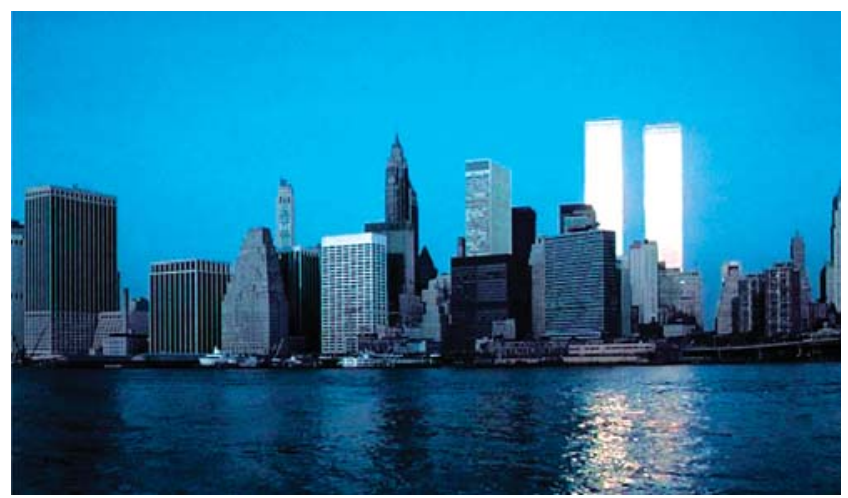

from the macro to the micro, in three of the Laboratory's Divisions (Materials Reliability, Metallurgy, and Polymers). The scope of activities includes the development and innovative use of state-of-the-art measurement systems; leadership in the development of standardized test procedures and traceability protocols; development of an understanding of materials in novel conditions; and development and certification of Standard Reference Materials (SRMs). Many of the tests involve extreme conditions, such as high rates of loading, high temperatures, or unusual environments (e.g., deep underwater). These extreme conditions often produce physical and mechanical properties that differ significantly from the handbook values for their bulk properties under traditional conditions. These objectives will be realized through innovative materials, property measurement and modeling.

The MSEL Safety and Reliability Program is also contributing to the development of test method standards through committee leadership roles in standards development organizations such as the ASTM International and the International Standards Organization (ISO). In many cases, industry also depends on measurements that can be traced to NIST Standard Reference Materials $\left(\mathrm{SRM}^{\circledR}\right)$.

In addition to the activities above, all three divisions provide assistance to various government agencies on homeland security and infrastructural issues. Projects include assessing the performance of structural steels as part of the NIST World Trade Center Investigation, advising the Bureau of Reclamation on metallurgical issues involving pipelines and dams, advising the Department of the Interior on the structural integrity of the U.S.S. Arizona Memorial, and collaborating with both the Department of Transportation and the Department of Energy on pipeline safety issues.

Contact: Frank W. Gayle (Metallurgy Division) 


\section{Analysis of Structural Steel in the World Trade Center}

In 2002 NIST became the lead agency in the investigation of the World Trade Center collapse. The investigation addresses many aspects of the catastrophe, from occupant egress to factors affecting how long the Twin Towers stood after being hit by the airplanes, with a goal of gaining valuable information for the future. A critical aspect of the investigation is the metallurgical analysis of structural steels from the Twin Towers. The analysis includes characterization of properties, failure modes, and temperature excursions seen by the steel.

\section{Stephen W. Banovic, Richard J. Fields,} Timothy J. Foecke, William E. Luecke, J. David McColskey, Christopher N. McCowan, Thomas A. Siewert, and Frank W. Gayle

$\mathrm{T}^{\mathrm{h}}$ he collapse of the World Trade Center (WTC) Towers on September 11, 2001, was the worst building disaster in human history. Engineers, emergency responders, and the nation were largely unprepared for such a catastrophe. NIST is investigating the disaster (see http://wtc.nist.gov/), and a primary objective is to determine why and how the towers collapsed after the initial impact of the aircraft. As part of this investigation, the Metallurgy and Materials Reliability Divisions in MSEL are studying recovered structural steel from the WTC site. Progress in this study is outlined here.

Task 1 - Collect and catalog physical evidence: 236 structural pieces of the WTC towers have been collected, brought to NIST and studied. Reports have been issued documenting the steel, the as-built locations, the structure of the towers based on design documents, and the standards at the time of construction.

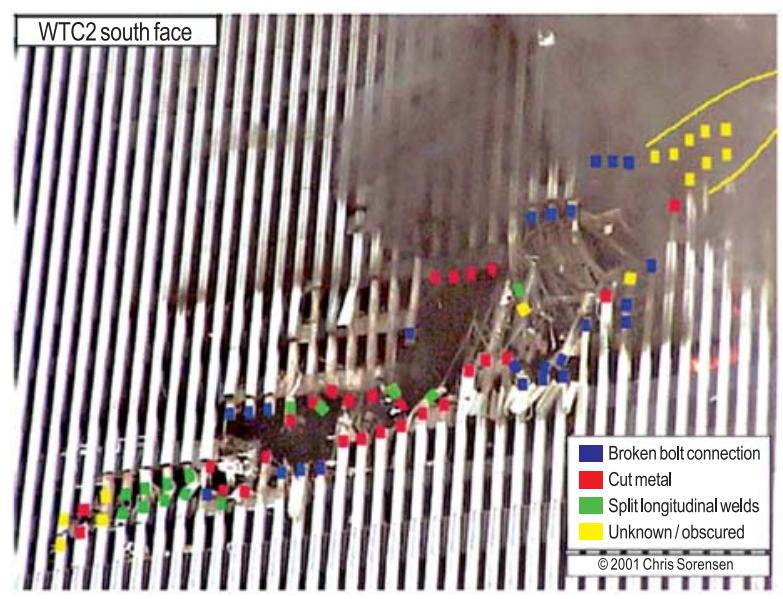

Figure 1: Enhanced image of the impact zone of WTC 2 has allowed determination of failure modes in the perimeter columns.
Task 2 - Categorize failure mechanisms based on visual evidence: Available photographic evidence and recovered steel have been examined and documented as to failure mechanisms (Figure 1).

Task 3 - Determine steel properties to support structure performance and airplane impact modeling studies: 29 different steels have been characterized for room temperature mechanical properties. High-temperature and high-strain-rate tests are now complete. Creep, or time-temperature-dependent behavior, has been determined for floor truss and column steel.

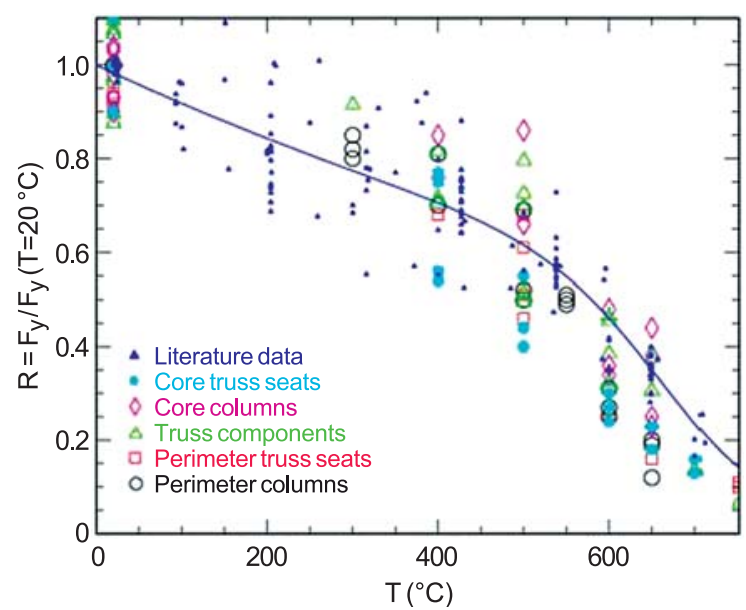

Figure 2: Normalized yield strength and ultimate tensile strength measured as function of temperature. Model curve is based on historical data for WTC era steels.

Task 4 - Correlate determined steel properties with specified properties: Most steel was found to exceed the specified minimum values by 5 to 10 percent.

Task 5 - Analyze steel to estimate temperature extremes: We have developed a technique of paint characterization to provide a quick mapping of temperature excursions seen by the steel. Challenges included deciphering pre- and post-collapse exposure (see related Highlight in this report).

Data on material properties have been provided to aid in the modeling of plane impact and structural response to the fires. A final draft of the investigation report will be released for public comment in December 2004.

\section{Contributors and Collaborators}

R. Santoyo, L. Rodine (Materials Reliability Division, MSEL, NIST); M. Williams, S. Claggett, M. Iadicola, R. Jiggetts (Metallurgy Division, MSEL, NIST) 


\section{Standard Test Methods for Fire-Resistant Steel}

\section{The fires in the World Trade Center and the} ensuing collapse focused attention on the vulnerability of structural steel to fire. Recently, steel mills in Europe and Japan have begun to market steels designated as "fire-resistant." This project is developing a standard test method for quantitatively evaluating and comparing the resistance to high-temperature deformation and failure of structural steels.

\section{William E. Luecke, J. David McColskey, Richard J. Fields, and Frank W. Gayle}

\begin{abstract}
$\mathrm{A}^{\mathrm{t}}$ room temperature, the stress-strain behavior of structural steel is considered to be independent of time. Above about $300{ }^{\circ} \mathrm{C}$, structural steel begins to lose strength. At still higher temperatures, about $500{ }^{\circ} \mathrm{C}$, creep, or time-dependent deformation, further reduces the load-carrying capability. By $600{ }^{\circ} \mathrm{C}$, most structural steels have lost more than half their strength. For this reason, structural steels in buildings are insulated to minimize the temperature rise and resulting strength loss.
\end{abstract}

Fire resistant (FR) steels are intended to be drop-in replacements for structural steel. They typically meet the same specifications with similar welding properties and cost only marginally more. Yet these steels retain superior high-temperature strength, offering potential for extra time for building occupants to escape a fire.

In Japan and Europe, FR steels are qualified based on yield strength retention at high temperature. This definition may not be the most appropriate since retained yield strength is a short-time property, yet fire-resistance is often measured in hours. Also, with increasing temperature, strength becomes increasingly sensitive to the strain rate. Current U.S. standards for load-bearing components in fire are based on time to reach a given temperature, so effectively all steels are considered to have the same high-temperature strength reduction, regardless of actual properties.

We are currently studying a hybrid of creep and conventional tension tests. The test specimen is held under constant load as the test temperature ramps upward linearly. Over a narrow temperature range, which can be approximated as a critical temperature, the deformation rate increases dramatically, and the specimen fails. This critical temperature can be used as a measure of fire resistance.

One goal of this project is to refine this test technique and offer it as a draft standard. Published research has established that the temperature ramp test can rank steels, but definitive studies comparing ordinary and FR steels are lacking. Until a standard test method is developed, it is difficult to confidently compare results between laboratories. This research will focus on understanding the limitations, repeatability, and reproducibility of the method by characterizing several different classes of construction steels. Interlaboratory studies will be used to probe the method's limitations, precision and bias.

A second goal is to generate constitutive models for modeling high-temperature deformation of steels. If test results are not predictable from underlying deformation behavior, a test cannot possibly indicate real-world performance. A second benefit is that much-needed data will be supplied for finite element modeling of modern structural steel deformation in fire, a prerequisite for performance-based fire resistant design.

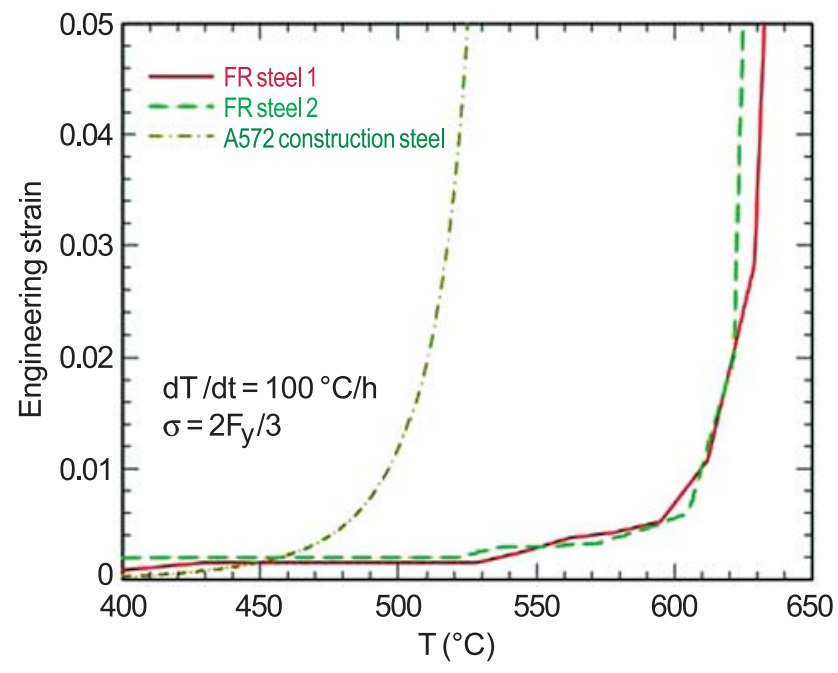

Figure 1: Comparison of "run-away creep" deformation of FR and ordinary construction steel.

Figure 1 illustrates the concept of critical temperature for "run-away" creep for two FR steels and one conventional construction steel. The results demonstrate the potential performance advantage of FR steel. Much more work is necessary, however, to understand the variability in performance of ordinary and FR steels.

During 2004, we also spearheaded the development of an ASTM task group under committee A01 to evaluate test methods for fire resistance of structural steel.

\section{Contributors and Collaborators}

\author{
M. Bykowski (SURF)
}




\section{Pipeline Safety: Corrosion, Fracture, and Fatigue}

A critical element of the nation's infrastructure is the more than 2 million miles of natural gas and hazardous liquid pipelines that provide over half of the nation's energy. While pipelines are the safest means of transporting these materials, they are not immune to failures. In 2002, Congress passed the Pipeline Safety Improvement Act to reduce pipeline failure rates, increase public safety, and ensure a continuous and uninterrupted delivery of the nation's energy supply. NIST is working with the pipeline industry, DoT, and DoE to provide the measurement methods, standards, and data needed to accomplish this objective.

\section{Richard E. Ricker}

Tn December 2002, Congress passed the Pipeline Safety Improvement Act (PSIA), through which the DoT Office of Pipeline Safety (DoT/OPS), DoE, and NIST will coordinate pipeline R\&D. In FY 2004, DoT/OPS, DoE, and NIST signed a memorandum of understanding (MOU) and a Five-Year Joint R\&D Plan detailing agency responsibilities in a program of research, development, demonstration, and standardization for pipeline integrity. The Department of the Interior/MMS has also been included in the plan because of its responsibility for off-shore oil pipelines. Under these agreements, NIST is responsible for materials research, fire safety, and standards development that pertain to pipeline safety, reliability, and damage prevention.

A number of factors have combined to increase the need for improvements in the safe operation of pipelines. Among these are increases in national energy needs,

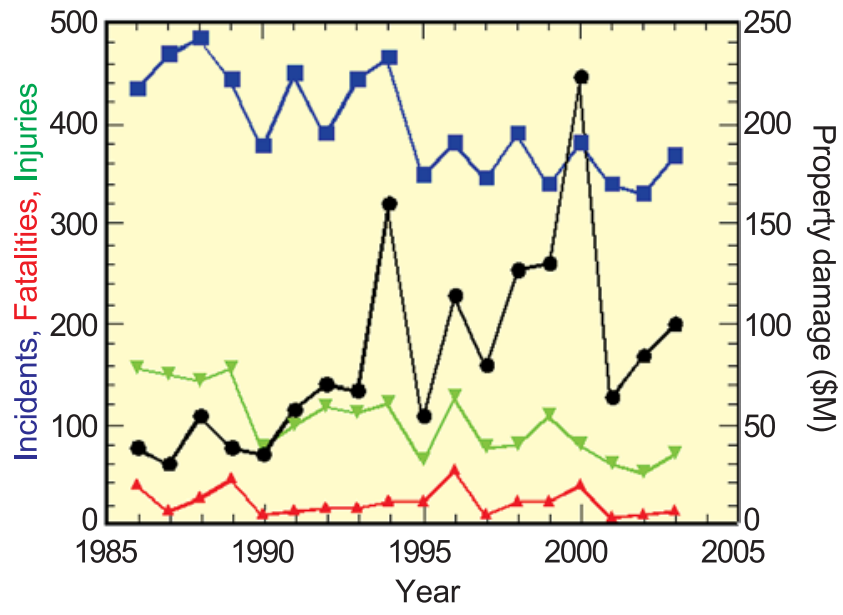

Figure 1: Pipeline incidents and damage since 1986 (DoT/OPS). population densities near pipelines, environmental concerns, and average pipeline age. While recent accidents have brought increased attention to this issue, pipeline incident rates have not changed significantly since 1986 (Figure 1). While failure mechanisms are not always thoroughly documented, the DoT/OPS incident data can be used to estimate the distribution of failure mechanisms (Figure 2). Clearly, fracture, fatigue and corrosion account for a significant proportion of the failures. Understanding and developing better standards for preventing these materials-related failure mechanisms is the objective of NIST R\&D in 2004-2005 project.

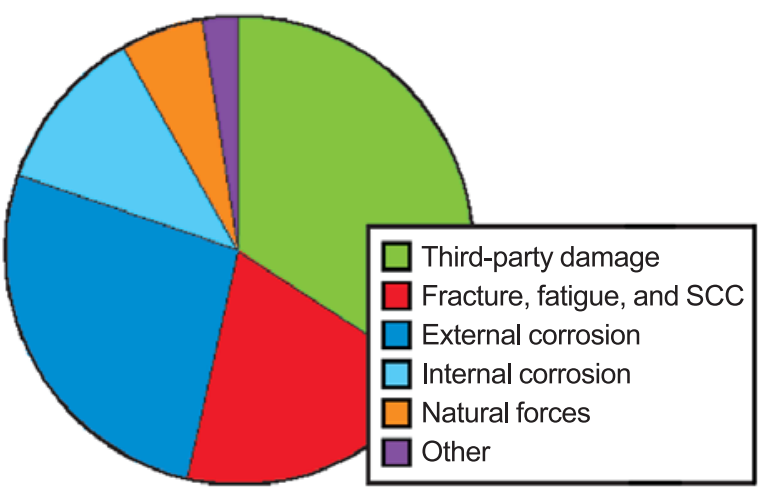

Figure 2: Estimated pipeline failure mechanism distribution based on DoT/OPS data over $\approx 10$ years.

NIST has a history of contributions to pipeline safety. For example, NIST conducted welding and fracture research for the Alaskan pipeline, and the NIST 14-year study of underground corrosion is the basis for several current pipeline standards. The current program will review existing standards, the current corrosion, fatigue, and fracture issues, and evaluate where improvements may be possible. This work will be done in collaboration with industry, particularly the Pipeline Research Council International (PRCI) Corrosion and Inspection Technical Committee, and with private sector consensus standards setting organizations. NIST and its collaborators have discussed and reviewed NIST R\&D plans and coordination. A Government/Industry Pipeline R\&D Forum is being organized for April 2005 to present the Five-Year Joint R\&D Plan.

\section{Contributors and Collaborators}

R.J. Fields, T.J. Foecke, F.W. Gayle, C.A. Handwerker (Metallurgy Division, NIST); T. Siewert, C. McCowan (Materials Reliability Division, NIST); C. Sames (PRCI); J. Merritt, S. Gerard, R. Smith (DoT/OPS); C. Freitas, R. Anderson, D. Driscoll (DoE); M. Else (DoI) 


\section{Frangible Bullets and Soft Body Armor}

Police officers' lives often depend upon the effectiveness of their soft body armor. Continued introduction of new types of body armor and bullets (including lead-free frangible models) are overwhelming the enforcement community's ability to test everything adequately. At the request of the Office for Law Enforcement Standards (OLES), we are conducting a thorough study to identify the determining factors for effective protection against the various types of bullets on the market.

\section{Lyle E. Levine and Richard Rhorer (822)}

$\mathrm{T}_{\mathrm{r}}^{\mathrm{h}}$ he impact of a bullet on soft body armor is a remarkably complex process involving complex geometries (including the density and weave pattern of the body armor fibers) and high-strain-rate properties of the bullet and armor materials. Complicating the issue further, recent lead-free bullet designs are being produced with a wide range of materials (copper, tin, iron, tungsten, zinc and polymers) and processing conditions (sintering, cold-pressing and injection molding). The mechanical behavior of these new bullet designs can be dramatically different from those of the conventional lead bullets for which soft body armor has been designed. Degradation of body armor materials with age, mechanical wear and exposure conditions (moisture and light) is also important. A long-term, comprehensive project to address all of these issues was started this fiscal year.

Planned tasks for bullets include:

- High-strain-rate testing of bullets (longitudinal and diametral) and bullet materials using the NIST Kolsky bar facility. Approximately 600 tests will be required.

- Corresponding low-strain-rate testing using a conventional mechanical testing machine.

- Metallographic and chemical analyses of bullet materials.

Planned tasks for soft body armor materials include:

- Design and construction of a miniature Kolsky bar for high-strain-rate testing of fibers.

- Design and construction of tensile cages for miniature and existing Kolsky bars.

- Exposure of fiber test samples to degrading conditions (light, moisture and abrasion)
- High-strain rate testing of as-received and degraded individual fibers, multiple non-braided fibers, and braided fiber bundles. Approximately 2000 tests will be required.

- Corresponding low-strain-rate testing using a conventional mechanical testing machine.

These tasks will be complemented by finite element modeling of:

- High-strain-rate bullet tests using measured high-strain-rate behavior of bullet materials.

- Fiber and fiber bundle tests using measured high-strain-rate behavior of fiber materials.

- Bullet impacts with woven body armor fabric.

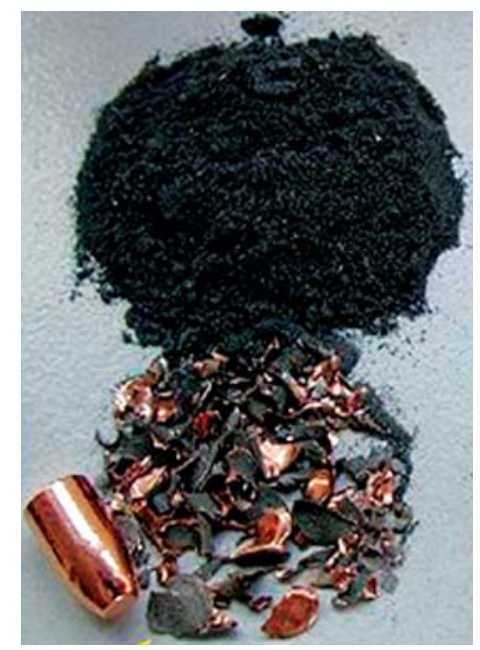

Figure 1: Frangible $9 \mathrm{~mm}$ bullet and remains after impact.

\section{Expected Impacts}

The test data will be used by the U.S. Department of Justice to determine what actions are required to ensure that soft body armor provides adequate protection to police officers in the field. The experimentally validated finite element models will be used to screen combinations of bullets and body armor designs to identify potential threats at an early stage.

\section{Contributors and Collaborators}

S. Banovic, R. Fields, L. Ma, S. Mates

(Metallurgy Division, NIST); M. Kennedy, E. Whitenton (Manufacturing Metrology Division, NIST); T. Burns (Mathematical and Computational Sciences Division, NIST) 


\section{Marine Forensics and Preservation of Historic Shipwrecks}

\begin{abstract}
Historic shipwrecks are valuable cultural resources continuously under attack by their environment. Preservation techniques aimed at slowing corrosion processes and mechanically stabilizing shipwreck structures are being modeled and designed in coordination with the National Park Service, NOAA, and the U.S. Navy. These techniques are being applied to such wreck sites as the USS Arizona, the Ellis Island Ferry, RMS Titanic, CSS Hunley, and the USS Monitor.
\end{abstract}

\section{Tim Foecke}

Since the attack on Pearl Harbor on December 7th, N1941, the wreck of the USS Arizona has been slowly corroding and collapsing. The monument that straddles the wreck sees over 700,000 visitors per year, and there is immense interest in preserving the wreck for as long as possible. Aside from the historical interest, there are substantial environmental risks associated with any large-scale collapse of the wreck, as there is still an estimated 500,000 gallons of fuel oil trapped below water.

The Submerged Cultural Resources Unit (SCRU) of the National Park Service (NPS) has responsibility for maintaining and preserving the wreck. Based on our past experience in marine forensics, SCRU approached the Metallurgy Division for assistance in several areas.

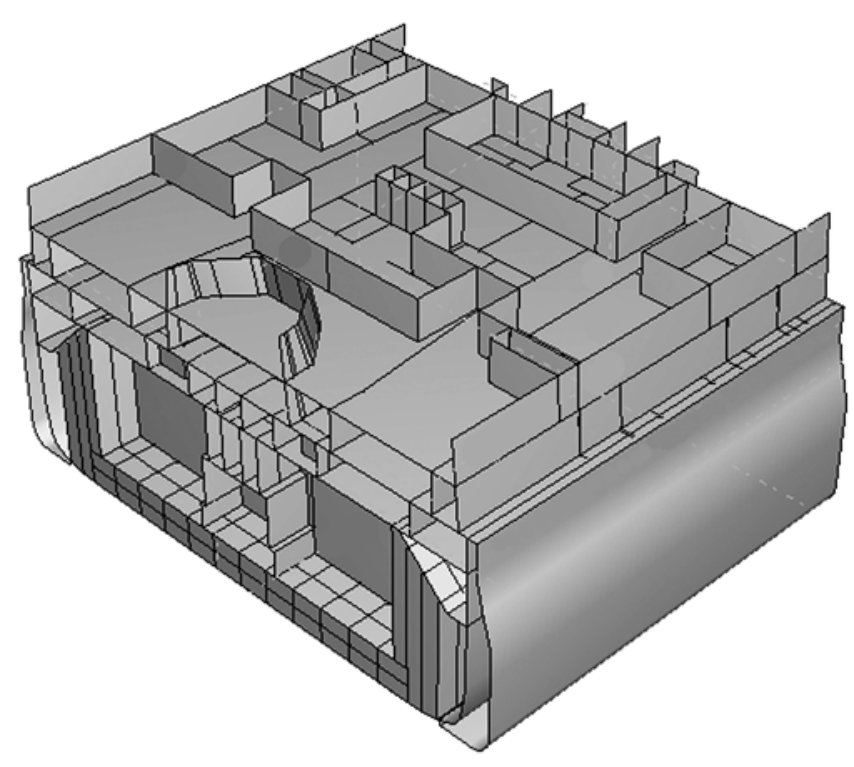

Figure 1: Finite element model of the center section of the USS Arizona, where fuel oil is believed to be concentrated.
Advice is being given on methods to measure the remaining thickness of the hull through the existing bioencrustation. A finite element model (Figure 1) is under construction, and techniques are being developed to map the corrosion current data gathered by the NPS over the last 20 years onto the model in the form of decreasing plate thicknesses.

The tools developed in this project will eventually be transferred to companies and organizations that are responsible for monitoring submerged wrecks containing hazardous materials, allowing them to better simulate critical conditions.

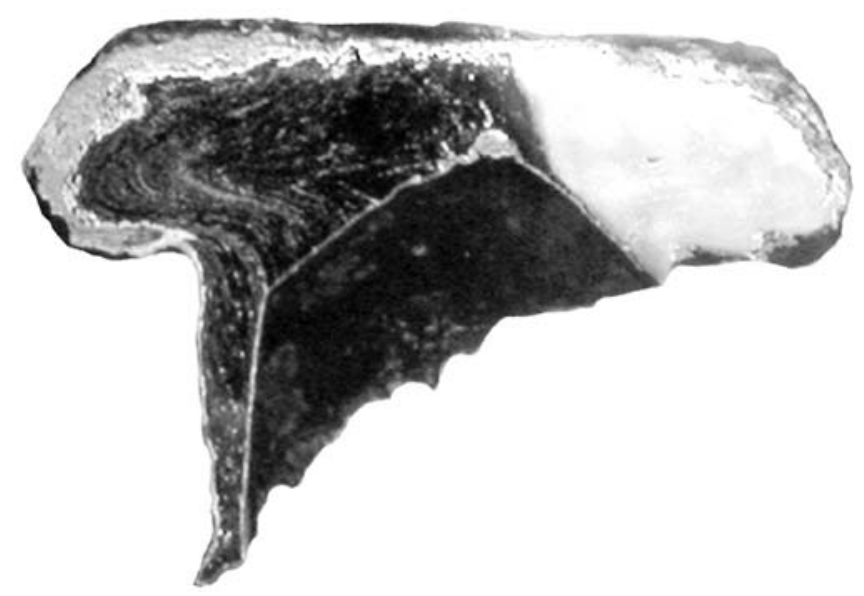

Figure 2: Cross-section of a rivet extracted from the submarine HL Hunley, showing slag flow lines on the left. The submarine, the first to sink an enemy ship, is currently on shore in Charleston, SC).

In addition to the USS Arizona, we are providing assistance and technical expertise to NPS, NOAA and the Navy regarding preservation of the Ellis Island Ferry, the CSS Hunley (Figure 2), the USS Monitor (partially salvaged), and the RMS Titanic. NIST participated in a multi-agency remote survey of the Titanic wreck in June 2004 at the University of Rhode Island, satellite linked to the NOAA Ship Ron Brown. Ongoing analysis of the photos and videos taken during this expedition will attempt to map the degradation of the wreck and chart the impact of any human visits.

\section{Contributors and Collaborators}

L. Ma, S. Banovic (Metallurgy Division, NIST); L. Murphy, M. Russell, D. Conlin (Submerged Cultural Resources Unit, National Park Service); Lt. J. Weirich (NOAA); Cdr. J. Morganthou (CINCPAC, U.S. Navy) 


\section{Kinetic Penetrators and Amorphous Metals}

\begin{abstract}
Environmental and political debate on the continued use of depleted uranium (DU) alloys has led to increased efforts at developing low-cost, reduced-toxicity replacement penetrator materials with similar ballistic performance. The Metallurgy Division has been working with the Army Research Laboratory (ARL) on a novel tungsten-amorphous metal composite material as one of these new $D U$ alloy replacements. The project relies on the Metallurgy Division's expertise in rapid solidification processing and instrumented powder consolidation to provide these composite materials to the ARL for ballistic testing.
\end{abstract}

\section{Stephen D. Ridder}

$\mathrm{B}$ ulk amorphous metal (BAM) alloys are a relatively new class of alloys that solidify amorphously in castings with cross-sections as large as $10 \mathrm{~mm}$ across, a significant improvement over the sub- $1 \mathrm{~mm}$ castings of conventional amorphous metal alloys. The Army's interest in high-density BAM alloys is a result of their need for a new armor-piercing projectile material to replace the current Depleted Uranium technology.

Modern, tank-fired armor-piercing munitions are high-velocity projectiles machined from DU alloys or from two-phase, tungsten-based heavy alloy (W-) composites. High-density materials are chosen to maximize the kinetic energy available for armor penetration. The projectile material is partially consumed by erosion or back extrusion as it enters armor plate. Current DU alloys are superior to available W-composites due to "continuous tip sharpening" compared to the tip blunting and "mushrooming" seen in the W-composites. This tip sharpening is generally attributed to adiabatic shear localization (ASL) and failure, a specific failure mode exhibited by DU alloys when rapidly loaded. Heat generated within the penetrator during high-strain-rate loading produces sufficient thermal softening of the DU alloy structure to overcome work-hardening in ASL bands concentrated near the tip of the penetrator that promotes rapid removal of deformed material. The continuously sharpened penetrator concentrates the remaining kinetic energy at the end of a relatively narrow tunnel, thus reducing energy loss and improving penetrator efficiency.

Recent work by the ARL has shown that BAM alloys have promise in providing the necessary
ASL-like behavior that could lead to a W-composite penetrator material with performance similar to DU alloys.

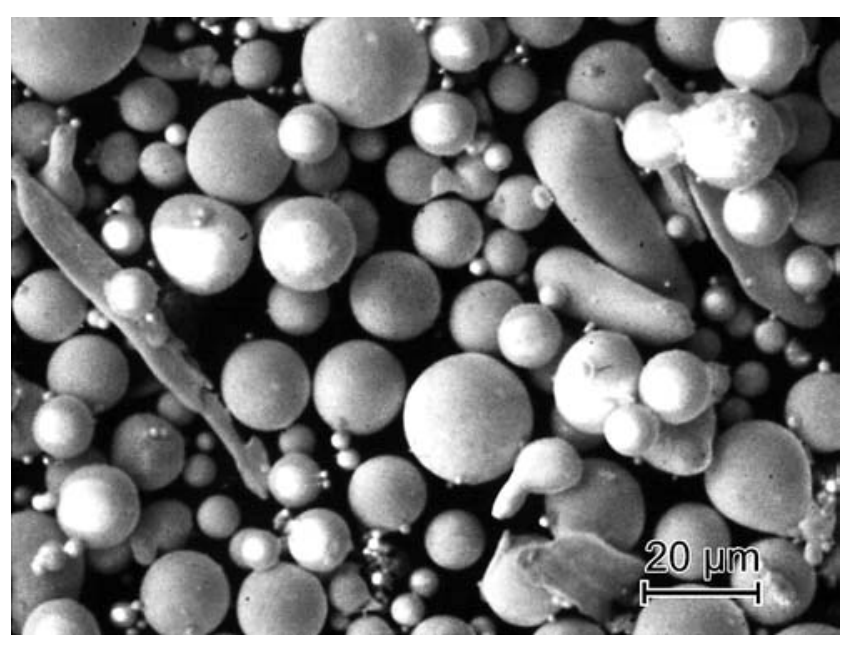

Figure 1: Amorphous Hf alloy powder particles produced in NIST small-scale gas atomization facility.

The Metallurgy Division designed and built a small-scale gas atomizer to study the composition-dependent solidification behavior of BAM alloys using small batch $(250 \mathrm{~g})$ processing to reduce material and processing costs. Figure 1 shows spherical amorphous Hf alloy powder particles recently produced. The material produced by this device is preferred to the more readily available comminuted melt-spun amorphous ribbon material because these spherical particles can be blended with tungsten powder and consolidated to produce a more homogeneous $\mathrm{W}$-composite billet.

A hot isostatic press (HIP) has been instrumented with an eddy current sensor developed at NIST to follow the composite consolidation and provide the necessary quantitative, real-time monitoring of the progress of densification. This technology will allow full densification of the W-composite without inducing large-scale crystallization of the Hf based BAM powder.

\section{Contributors and Collaborators}

F.S. Biancaniello, C.E. Campbell, R.J. Fields, R.D. Jiggetts, U.R. Kattner, S.P. Mates (NIST), L. Kecskes (U.S. Army) 



\section{Metallurgy Division FY04 Annual Report Publication List}

\section{Anelasticity and Springback Prediction}

Ma, L., T.F. Zahrah, and R.J. Fields, "Numerical three dimensional simulation of cold compaction and springback for particulate prealloyed reinforced composites," Powder Metallurgy Journal 47 (1), 31-36, 2004.

Munitz, A., D. Dayan, D.J. Pitchure, and R.E. Ricker, "Dynamic mechanical analysis of pure Mg and Mg AZ31 alloy," Magnesium Technology 2004, edited by A.A. Lu, TMS, 2004.

Paul, R.L., H. Chen-Mayer, G.R. Myneni, W.A. Lanford, and R.E. Ricker, "Hydrogen uptake by high purity niobium studied by nuclear analytical methods," Matériaux and Techniques 91 (7-8-9), 23-27, 2003.

\section{Microstructural Origins of Surface Roughening and Strain Localization}

Liu, J., S.W. Banovic, F.S. Biancaniello, and R.D. Jiggetts, "Through-thickness texture inhomogeneity in a recrystallized Al-Mg alloy," Met. Trans. A Communications, (submitted), 2004.

Liu, J., and J.G. Morris, "Recrystalliation microstructures and textures in AA 5052 continuous cast and direct chill cast aluminum alloy," Materials Science Eng A, (submitted), 2004.

Stoudt, M.R., S.W. Banovic, and T. Quarrick, "Evolution of deformation-induced surface morphologies developed on Fe-based sheet metal," 45th MWSP Conference Proceedings, edited by M.A. Baker, Warrendale, PA: Iron and Steel Society, pp. 507-517, 2003.

Stoudt, M.R., J.B. Hubbard, and S.W. Banovic, "Evolution of deformation induced surface morphologies generated in Fe-based sheet," Test and Measurement World, (submitted), 2004.

Stoudt, M.R., J.B. Hubbard, and S.W. Banovic, "Evolution of deformation induced surface morphologies generate in Fe-based sheet," Special Publication \# 1837; Innovations in Modeling and Testing of Steel Structures for Automotive Applications, edited by R. Mohan, et al., Warrendale, PA: SAE, 2004.

Stoudt, M.R., J.B. Hubbard, and S.W. Banovic, "Evolution of deformation induced surface morphologies generated in Fe-based sheet," Transactions of the SAE, (submitted), 2004.
Stoudt, M.R., J.B. Hubbard, and S.W. Banovic, "Evolution of deformation induced surface morphologies generated in Fe-based sheet," SAE Transactions: Journal of Materials and Manufacturing, (in press), 2004.

Stoudt, M.R., A. Munitz, S.W. Banovic, and R.J. Fields, "Effect of uniaxial strain on the surface roughness of pure Mg and Mg AZ31 alloy," Magnesium 2004, (submitted), 2004.

Stoudt, M.R., A. Munitz, S.W. Banovic, and R.J. Fields, "Effect of uniaxial strain on the surface roughness of pure Mg and Mg AZ31 alloy," Magnesium Technology 2004, edited by A.A. Luo, Warrendale, PA: TMS, pp. 269-274, 2004.

\section{Underlying Processes of Plastic Deformation in Metal Alloys}

Cai, M., S.C. Langford, L.E. Levine, and J.T. Dickinson, "Determination of strain localization in aluminum alloys using laser-induced photoelectron emission," Journal of Applied Physics, (submitted), 2004.

Delos-Reyes, M.A., M.E. Kassner, and L.E. Levine, "X-ray diffraction and the existence of long range internal stresses," Dislocations, Plasticity and Metal Forming, edited by A.S. Khan, R. Kazmi, and J. Zhou, Fulton, MD: Neat Press, p. 262, 2003.

Khan, S.M, H.M. Zbib, and D.A. Hughes, "Multi-scale dislocation dynamics plasticity: application for Frank's dislocation boundaries," (submitted), 2004.

Kramer, D.E., M.F. Savage, and L.E. Levine, "AFM observations of slip band development in $\mathrm{Al}$ single crystals," Acta Materialia, (submitted), 2004.

Levine, L.E., "Multiscale phenomena in materials experiments and modeling related to mechanical behavior," Symposium Proceedings Vol. 779, edited by H.M. Zbib, D.H. Lassila, L.E. Levine, and K.J.Hemker, Warrendale, PA: MRS, 2003.

Levine, L.E., and R.M. Thomson, "A statistical connection between dislocations and mechanical properties," Dislocations, Plasticity and Metal Forming, edited by A.S. Khan, R. Kazmi, and J. Zhou, Fulton, MD: Neat Press, p. 286, 2003.

Tavazza, F., L.E. Levine, and A. Chaka, "Atomistic insights into dislocation dynamics in metal forming," Proceedings of 3rd International Computational Modeling and Simulation of Materials, Acireale, Italy, (in press), 2004. 


\section{High Speed Machining}

Basak, D., H.W. Yoon, R. Rhorer, T.J. Burns, and T. Matsumoto, "Temperature control of pulse heated specimens in a Kolsky bar apparatus using microsecond time-resolved pyrometry," International Journal of Thermophysics, 25 (2), 561-574, 2004.

Basak, D., H.W. Yoon, R. Rhorer, and T.J. Burns, "Microsecond time-resolved pyrometry during rapid resistive heating of samples in a Kolsky bar apparatus," American Institute of Physics, 684, Part 2, pp. 753-757, 2003.

Burns, T., M. Davies, R. Rhorer, D. Basak, H. Yoon, R.J. Fields, L.E. Levine, E. Whitenton, M. Kennedy, and R. Ivester, "Influence of heating rate of flow stress in modeling of machining processes," Proceedings of the CIRP International Workshop on Modeling and Machining, Cluny, France, (in press), May 2004.

\section{Mechanisms for Delivery of Thermodynamic and Kinetic Data}

Campbell, C.E., W.J. Boettinger, T. Hansen, P. Merewether, and B.A. Mueller, "Examination of multicomponent diffusion between two Ni-base superalloys," Properties of Complex Inorganic Solids 3, edited by P. Turchi, et al., New York: Kluwer Academics/Plenum Publishers, (in press), 2003.

Campbell, C.E., J.C. Zhao, and M.F. Henry, "Comparison of experimental and simulated multicomponent Ni-base superalloy diffusion couples," Journal of Phase Equilibria and Diffusion, 25 (1), 6-15, 2004.

Kattner, U.R., J.E. Morral, and W.J. Boettinger, "Databases for computational thermodynamics and diffusion modeling," Journal of Phase Equilibria and Diffusion, 24 (3), 416-421, 2003.

\section{Phase Field Modeling Tools for Microstructure and Stress Evolution}

Alikakos, N.D., P.W. Bates, J.W. Cahn, P.C. Fife, G. Fusco, and G.B. Tangoglu, "Extreme Anisotropy of Diffusion Interfaces in Crystals," SIAM Journal of Applied Mathematics, (in press), 2003.

Cahn, J.W., and J. Taylor, "A unified approach to motion of grain boundaries, relative tangential translation along grain boundaries, and grain rotation," Acta Materialia, (in press), 2003.

Granasy, L., T. Pusztai, T. Borzsonyi, J.A. Warren, and J.F. Douglas, "A general mechanism of polycrystalline growth," Nature Materials, (in press), 2004.
Granasy, L., T. Pusztai, G. Tegze, J.A. Warren, and J.F. Douglas, "On the growth and form of spherulites," NATURE, (submitted), 2004.

Granasy, L., T. Pusztai, and J.A. Warren, "Modelling polycrystalline solidification using phase field theory," Journal of Physics: Condensed Matter, (in press), 2004.

Guyer, J.E., W.J. Boettinger, J.A. Warren, and G.B. McFadden, "Phase Field Modeling of Electrochemistry I: Equilibrium," Physical Review E, 69 (2) Art. No. 021603, Part 1, February 2004.

Guyer, J.E., W.J. Boettinger, J.A. Warren, and G.B. McFadden, "Phase Field Modeling of Electrochemistry II: Kinetics," Physical Review E, 62 (2), Art. No. 02164, 2004.

Guyer, J.E., D. Wheeler, and J.A. Warren, "A finite volume PDE solver using Python (FIPy)," http://www.python.org/dc2004/papers/28, WEB Publication, (submitted), 2004.

Lewis, D.J., T. Puztai, L. Granasy, J.A. Warren, and W.J. Boettinger, "Phase field models for eutectic solidification," Journal of Materials, 56 (4), 34-39, April 2004.

Sekerka, R.F., and J.W. Cahn, "Solid-liquid equilibrium for non-hydrostatic stress," Acta Materialia, (submitted), 2003.

Tegze, G., J.A. Warren, L. Granasy, T. Puztai, T. Borzsonyi, and J.F. Douglas, "The influence of foreign particles in the formation of polycrystalline solidification patterns," Solidification Processes and Microstructures: A Symposium in Honor of Prof. W. Kurz, M. Rappaz, C. Beckermann, and R. Trivedi, TMS, pp. 379-385, 2004.

Upmanyu, M., D.J. Srolovitz, A.E. Lobkovsky, J.A. Warren, and W.C. Carter, "Simultaneous grain boundary migration and grain rotation," Acta Materialia, (submitted), 2003.

Warren, J.A., R. Kobayashi, A.E. Lobkovsky, and W.C. Carter, "Extending phase field models of polycrystalline materials," Acta Materialia, 51, 6035-6058, 2003.

Warren, J.A., L. Loginova, L. Granasy, T. Borzsonyi, and T. Pusztai, "Phase field modeling of alloy polycrystals," Proceedings for Modeling Casting and Advanced Solidification Processes X, edited by D. Stefanescu, J.A. Warren, M. Krane, and M. Jolly, p. 45-52, 2003. 


\section{Hardness Standardization - Rockwell, Vickers, Knoop}

Ma, L., S. Low, and J. Fink, "Effect of steel and tungsten carbid ball indenters on Rockwell hardness tests," Proceedings of HARDMEKO, (submitted), 2004.

Stibler, A., S.R. Low, and R. Ellis, "Comparative measurements of Slovenian and USA Rockwell C hardness scales," Hardmeko 2004, (submitted), 2004.

\section{D Electron Microscopy}

Ford, A.X., J.E. Bonevich, R.D. McMichael, M.D. Vaudin, and T.P. Moffat, "Structure and magnetic properties of electrodeposited Co on n-GaAs(001)," Journal of Electrochemical Society, Vol. 150, Issue 11, pp. C753-759, September 2004.

Lehman, S.Y., A. Roshko, R.P. Mirin, and J.E. Bonevich, "Investigation of the shape of InGaAs/ GaAs quantum dots," Proceedings of Materials Research Society, Vol. 737, 2003, (in press).

\section{Nanostructure Fabrication Processes: Electrochemical Processing of Nanowires}

Kongstein, O., U. Bertocci, and G.R. Stafford, "In situ stress measurements during copper electrodeposition on (111)-textured Au," (submitted), 2004.

Wheeler, D., T.P. Moffat, G.B. McFadden, S.R. Coriell, and D. Josell, "Influence of a catalytic surfactant on roughness evolution during film growth," Journal of Electrochemical Society, 151 (8), C538-544, July 2004.

\section{USAXS Imaging of Biomaterials}

Levine, L.E., and G.G. Long, "X-ray imaging with ultra-small-angle $\mathrm{x}$-ray scattering as a contrast mechanism," Journal of Applied Crystallography, (in press), 2004.

\section{Measurements for Compound Semiconductors}

Davydov, A.V., L.A. Bendersky, W.J. Boettinger, D. Josell, M.D. Vaudin, C.S. Chang, and I. Takeuchi, "Combinatorial investigation of structural quality of $\mathrm{Au} / \mathrm{Ni}$ contacts on GaN," Applied Surface Science Journal 223 (1-3), 24-29, 2004.

Lu, C.J., L.A. Bendersky, H. Lu, and W.J. Schaff, "Suppression of threading dislocations in InN thin films grown on (0001) sapphire with a GaN buffer layer," Applied Physics Letters 83, 2817, 2003.
Lu, C.J., A.V. Davydov, D. Josell, and L.A. Bendersky, "Interfacial reactions of Ti/n-GaN contacts at elevated temperature," Journal of Applied Physic, Vol. 94, No. 1, pp. 245-253, 2003.

Schenck, P., D.L. Kaiser, and A.V. Davydov, "High-throughput characterization of the optical properties of compositionally graded combinatorial films," Applied Surface Science Journal, 223 (1-3), 200, 2004.

\section{Pb-free Surface Finishes: Sn Whisker Growth}

Bogustavsky, I., P. Bush, E. Kam-Lum, M. Kwoka, J. McCullen, K. Spalding, K. Vo, and M.E. Williams, "NEMI tin whisker test method standards," SMTAI Proceedings, (submitted), 2003.

\section{Pb-free Solder: Manufacturability and Reliability}

Allen, S.L., M.R. Notis, R.R. Chromik, R.P. Vinci, D.J. Lewis, and R.J. Schaefer, "Microstructural evolution in lead-free solder alloy Part II: directionally solidified eutectic tin-silver-copper, tin-copper and tin-silver alloys," Journal of Materials Research, (submitted), 2004.

Bertocci, U., "An EQMB examination of $\mathrm{Cu}$ surface oxides in borate buffer," Electrochemica Acta 49, 1831-1841, 2004.

Handwerker, Carol, Jasbir Bath, Elizabeth Bennedetto, Edwin Bradley, Ron Gedney, Tom Siewert, Polina Snugovsky, and John Sohn, NEMI Lead-Free Assembly Project: Comparison Between PbSn and $\mathrm{SnAgCu}$ Reliability and Microstructures, Benedetto, Proceedings of Surface Mount Technology International, Chicago, 2003.

Handwerker, C.A., U.R. Kattner, and K.-W. Moon, "Materials Science Concepts in Lead-Free Soldering," Pb-Free Solders, edited by K. Suganuma, Marcel Dekker, 2004.

Handwerker, C.A., E.E. deKluizenaar, K. Suganuma, and F.G. Gayle, "Major International Lead (Pb)-Free Solder Studies," Handbook of Lead-Free Solder Technology for Microelectronic Assemblies, edited by K. Puttlitz and K. Stalter, McGraw Hill/IEEE, pp. 665-728, 2004.

Moon, K., and W.J. Boettinger, "Difficulties in determining eutectic compositions: the $\mathrm{Sn}-\mathrm{Ag}-\mathrm{Cu}$ ternary eutectic," JOM 56 (4), 22-27, 2004. 
Rae, A., and C.A. Handwerker, "NEMI's Characterization of Lead-Free Alloy Applicable to Today's Commercially Available Alloys," Circuits Assembly, April 2004.

Schaefer, R.J., and D.J. Lewis, "Directional solidification in a $\mathrm{AgCuSn}$ eutectic alloy," Journal of Electronic Materials, (in press), 2003.

\section{Electrical Properties of On-Chip Interconnections}

Gayle, F., "FY2003 Programs and Accomplishments - MSEL Materials for Micro- and Optoelectronics, NISTIR 7019, 2004.

Josell, D., C. Burkhard, D. Kelley, Y.-W. Cheng, R.R. Keller, T.P. Moffat, Y. Li, B.C. Baker, C.A. Witt, and J.E. Bonevich, "Electrical properities of superfilled Sub-100 nm silver metallizations," Journal of Applied Physics, 96 (1), 759-768, 2004.

Josell, D., S. Kim, D. Wheeler, T.P. Moffat, and S.G. Pyo, "Quantifying superconformal filling of the submicrometer features through surfactant catalyzed chemical vapor deposition," Proceedings of 2003 Spring Meeting of Electrochemical Society in Paris, France, PV 2003-8, pp. 98-103, 2003.

Josell, D., T.P. Moffat, and D. Wheeler, "An exact, algebraic solution for the incubation period of superfill," Journal of the Electrochemical Society 151 (1), C19-C24, 2004.

Moffat, T.P., and D. Josell, "Electrodeposition of copper in the SPS-PEG-C1 additive system: I. kinetic measurements: influence of SPS," Journal of Electrochemical Society 151 (4), C262-C271, 2004.

Turchi, P.E.A., R.M. Waterstrat, R. Kuentzler, V. Drchal, and J. Kudrnovsky, "Electronic and phase stability properties of $\mathrm{V}-\mathrm{X}(\mathrm{X}=\mathrm{Pd}, \mathrm{Rh}, \mathrm{Ru})$ alloys," Journal of the Physics of Condensed Matter, (in press), 2004.

\section{Nanomagnetodynamics}

Lewis, A.C., D. Josell, and T.P. Weihs, "Stability in thin film multilayers and microlaminates: the role of free energy, structure, and orientation at interfaces and grain boundaries," Scripta Materialia 48 (8), 1079-1085, 2003.

McMichael, R.D., "A classical model of extrinsic ferromagnetic resonance linewidth in ultra-thin films," IEEE Transactions on Magnetics 40 (1), June 2004.

McMichael, R.D., "Ferromagnetic resonance linewidth models for perpendicular media," Journal of Applied Physics 95 (1), 1 June 2003.

\section{Magnetic Materials for Sensors and Ultra-high Density Data Storage}

Buchanan, J.D., T.P. Hase, B.K. Tanner, C.J. Powell, and W.F. Egelhoff, Jr., "Interface intermixing and in-plane grain size in aluminumtransition metal bilayers," Physical Review B, (submitted), 2004.

Chopra, H.D., Yang, D.X., Chen, P.J., and W.F. Egelhoff, Jr., "Domain structure and magnetoeleastic properttes of $\mathrm{Fe} / \mathrm{Pd}$ ferromagnetic thin films and multilayers," Journal of Applied Physics, (submitted), 2004.

Egelhoff, Jr., W.F., "Coercivities above 10 kOe in CoPd superlattices," Journal of Applied Physics, (submitted), 2004.

Egelhoff, Jr., W.F., L. Gan, H. Ettedgui, Y. Kadmon, C.J. Powell, P.J. Chen, A.J. Shapiro, R.D. McMichael, J. Mallet, T.P. Moffat, M.D. Stiles, and E.B. Svedberg, "Artifacts that mimic ballistic magnetoresistance," Journal of Magnetism and Magnetic Materials, (submitted), 2004.

Egelhoff, Jr., W.F., L. Gan, H. Ettedgui, Y. Kadmon, C.J. Powell, P.J. Chen, A.J. Shapiro, R.D. McMichael, J. Mallet, M.D. Stiles, and E.B. Svedberg, "Artifacts in ballistic magnetoresistance measurements," Journal of Applied Physics, 95, 7554, 2004.

Egelhoff, Jr., W.F., L. Gan, E.B. Svedberg, C.J. Powell, A.J. Shapiro, R.D. McMichael, J. Mallet, M.D. Stiles, and T.P. Moffat, "Artifacts that could be misinterpreted as ballistic magnetoresistance," Digest of PMRC 2004, p. 248, 2004.

Hong, J.I., S. Sankar, A.E. Berkowitz, and W.F. Egelhoff Jr., "Perpendicular anisotropy in $\mathrm{Co} / \mathrm{Pd}$ multilayers," Journal of Magnetism and Magnetic Materials, (submitted), 2004.

Huang, H., K. Seu, W.F. Egelhoff, Jr., and A. Reilly, "Ultrafast pump-probe laser spectroscopy of the half-metal $\mathrm{CrO}_{2}$," Journal of Applied Physics, (in press), 2004.

Lee, C.G., J.-G. Jung, V.S. Gornakov, R.D. McMichael, A. Chen, and W.F. Egelhoff, Jr., "Effects of annealing on the GMR and domain structure stabilization in a Py/Cu/Py/MnIr spin valve," Journal of Magnetism and Magnetic Materials 272, 1887, 2004.

Mallett, J.J., E.B. Svedberg, H. Ettedgui, T.P. Moffat, and W.F. Egelhoff, Jr., "A search for ballistic magnetoresistance (BMR) in electrodeposited Ni nanocontacts: feedback control used to select the contact resistance," Applied Physical Letters, (in press), 2004. 
Mallet, J., E.B. Svedberg, S. Sayan, A.J. Shapiro, P.J. Chen, W.F. Egelhoff, Jr., and T.P. Moffat, "Compositional control in electrodeposited CoPt films," Electrochemical Solid State Letters, (in press), 2004.

Misra, R.D.K., S. Gubbala, A. Kale, and W.F. Egelhoff, Jr., "A comparison of the magnetic characteristics of nanocrystalline $\mathrm{Ni}, \mathrm{Zn}$, and $\mathrm{Mn}$ ferrites synthesized by reverse micelle technique," Materials Science and Engineering, (in press), 2004.

Osofsky, M.S., R.J. Soulen, Jr., G. Woods, and W.F. Egelhoff, Jr., "Enhanced $\mathrm{T}_{\mathrm{c}}$ near the metal/insulator transition: new insight into novel superconducting materials," Physical Review Letters, (submitted), 2003.

Sanz, M., A. Papageorgopoulos, W.F. Egelhoff, Jr., M. Nieto-Vesperianas, and N. Garcia, "Wedge-shaped absorbing samples look left handed: the problem of interpreting negative refraction, and Its Solution," Physical Review E 67, Art. No. 067601, 2003.

Svedberg, E.B., J.J. Mallett, H. Ettedgui, L. Gan, P.J. Chen, A.J. Shapiro, N. García, T.P. Moffat, and W.F. Egelhoff, Jr., "Possible ballistic magnetoresistancelike artifacts in electrodeposited nanocontacts," Journal of Applied Physics, (in press), 2004.

Svedberg, E.B., J. Mallett, S. Sayan, A.J. Shapiro, W.F. Egelhoff, Jr., and T.P. Moffat, "Recrystallization texture and magnetic properties of electrodeposited FePt on Cu (001)," (submitted), 2004.

Woods, G.T., R.J. Soulen, Jr., I.I. Mazin, B. Nadgorny, M.S. Osofsky, J. Sanders, H. Srikanth, W.F. Egelhoff, Jr., and R. Datla, "Analysis of pointcontact Andreev reflection spectra in spin polarization measurements," Physical Review B, (in press), 2004.

Yang, D.X., H.D. Chopra, P.J. Chen, and W.F. Egelhoff, Jr., "Mixed-surfactants as a novel approach to atomic engineering of spin values," IEEE Transactions of Magnetics, (submitted), 2004.

Yang, D.X., E.J. Repetski, H.D. Chopra, B.J. Spencer, D.C. Parks, P.J. Chen, and W.F. Egelhoff, Jr., "A pinhole coupling strength in giant magnetoresistance spin valves: a statistical approach," Physical Review B, (in press), 2004.

\section{Electrodeposited $\mathrm{Pt}_{1-\mathrm{x}}(\mathrm{Fe}, \mathrm{Co}, \mathrm{Ni})_{\mathrm{x}}$ Alloys}

Mallet, J., E.B. Svedberg, S. Sayan, A.J. Shapiro, W.F. Egelhoff, Jr., and T.P. Moffat, "Compositional control in electrodeposition of FePt films," Electrochemical Solid State Letters, (submitted), 2004.
Zheng, L.A., E.V. Barrera, and R.D. Shull, "Magnetic properties of the Co-C(d60) and $\mathrm{Fe}-\mathrm{C}(\mathrm{d} 60)$ nanocrystalline magnetic thin films," Journal of Applied Physics, (submitted), 2003.

\section{Magnetic Properties and Standard Reference Materials}

Provenzano, V., J. Li, T.T. King, E.R. Canavan, P. Shirron, M.J. DiPorrp, and R.D. Shull, "Enhanced magnetocaloric effects in $\mathrm{R}_{3}\left(\mathrm{Ga}_{1-\mathrm{x}} \mathrm{Fe}_{\mathrm{x}}\right)_{5} \mathrm{O}_{12}$ $(\mathrm{R}=\mathrm{Gd}$, Dy, Ho; $(\mathrm{O}<\mathrm{x}<1)$ nanocomposites," Journal of Magnetism and Magnetic Materials 266, 185-193, 2003.

Provenzano, V., A.J. Shapiro, T.T. King, E.R. Canavan, P. Shirron, M. DiPirro, and R.D. Shull, "Peak magentocaloric effects in Al-Gd-Fe- alloys," Journal of Applied Physics, (submitted), 2004.

Provenzano, V., A.J. Shapiro, and R.D. Shull, "Near-elimination of large hysteresis losses in the Gd5Ge2S12 magnetic refrigerant compound by a small alloying addition of iron," Nature Journal, (submitted), 2004.

Shir, F., L.H. Bennett, E. Della Torre, C. Mavripis, L. Yanik, and R.D. Shull, "Details of the sequential steps in magnetocaloric regeneration," IEEE Transactions on Magnetics, (submitted), 2004.

Shir, F., E. Della Torre, L.H. Bennett, C. Mavripis, and R.D. Shull, "Modeling of magnetization and demagnetization in magnetic regenerative refrigeration," IEEE Transactions on Magnetics, (submitted), 2004.

Shir, F., E. Della Torre, L.H. Bennett, C. Mavripis, and R.D. Shull, "Modeling of magentization and demagnetization in magnetic regenerative refrigeration," IEEE Transactions of Magnetics, (submitted), 2004.

Shull, R.D., E. Quandt, and A.J. Shapiro, "MOIF observations of domain motion in magnetostrictive materials under stress," Journal of Applied Physics, (submitted), 2004.

Taketomi, S., R.V. Drew, and R.D. Shull, Anomalous magnetic aftereffect of a frozen magnetic fluid," Journal of Applied Physics, (submitted), 2004.

Taketomi, S., R.V. Drew, and R.D. Shull, "Magnetic fluids' phase transition between micelle and mono-dispersed phases," Physical Review E, (submitted), 2004.

Taketomi, S., and R.D. Shull, "Experimental verification of interactions between randomly distributed fine magnetic particles," Journal of Magnetism and Magnetic Materials, (in press), 2003. 
Turchinskaya, M.J., L.A. Bendersky, A.J. Shaprio, K.S. Chang, I. Takeuchi, and A.L. Roytburd, "Rapid constructing magnetic phase diagrams by magnetooptical imaging of composition spread films," Journal of Materials Research, (submitted), 2004.

\section{Analysis of Structural Steel in the World Trade Center}

Gayle, F.W., S.W. Banovic, T. Foecke, R.F. Fields, W.E. Luecke, J.D. McColskey, C. McCowan, and T.A. Siewert, "The Structural Steel of the World Trade Center Towers," Advanced Materials and Processes, (in press), October 2004.

Gayle, F.W., R.J. Fields, T.A. Siewert, and W.E. Luecke, "Interim Report on Contemporaneous Structural Steel Specifications," June 2004 Progress Report on the Federal Building and Fire Safety Investigation of the World Trade Center Disaster, NIST Special Publication 1000-5, pp. E-1 to E-61, 2004.

Banovic, S.W., "Interim Report on Inventory and Identification of Steels Recovered from the WTC Buildings," June 2004 Progress Report on the Federal Building and Fire Safety Investigation of the World Trade Center Disaster, NIST Special Publication 1000-5, pp. F-1 to F-64, 2004.

\section{Pipeline Safety}

Datta, P.K., H.L. Du, J.S. Burnell-Gray, and R.E. Ricker, "Corrosion of intermetallics," ASM Metals Handbook, Vol. 13, Corrosion, (in press), 2004.

\section{Kolsky Bar (OLES Project)}

Ma, L., J. Song, E. Whitenton, and T. Vorburger, "A bullet signatgure measurement system for NIST RM (Reference Material) 8240 standard bullets," Journal of Forensic Sciences 49 (4), 649-659, 2004.

Rhorer, R., D. Basak, G. Blessing, T. Burns, M. Davies, B. Dutterer, R.J. Fields, M. Kennedy, L.E. Levine, E. Whitenton, and H. Yoon, "Kolsky bar with electrical pulse heating of the sample," Proceedings for Society of Experimental Mechanics Annual Meeting, (submitted), 2003.

Rhorer, R.L., L.E. Levine, T.J. Burns, R.J. Fields, H.W. Yoon, M.A. Davies, D. Basak, E.P. Whitenton, G.V. Blessing, B.S. Dutterer, and M.D. Kennedy, "Constitutive model data for machining simulation using the NIST pulse-heated kolsky bar," Dislocations, Plasticity and Metal Forming, edited by A.S. Khan, R. Kazmi, and J.Zhou, Fulton, MD: NEAT Press, p. 103, 2003.
Yoon, H.W., D. Basak, R. Rhorer, E. Whitenton, T. Burns, R.J. Fields, and L.E. Levine, "Thermal imaging of metals in a kolsky-bar apparatus," Thermosense $X X V$, edited by E. Cramer and X.P. Maldague, Proceedings of SPIE, 5073, 284-294, 2003.

\section{Marine forensics}

Hooper, J.J., T.J. Foecke, L. Graham, and T.P. Weihs, "Metallurgical forensic analysis of wrought iron from the RMS titanic," Marine Technology, SNAME N40(2), pp. 73-81, April 2002.

\section{Kinetic Penetrators and Amorphous Metals}

Biancaniello, F.S., T.F. Zahrah, R.D. Jiggetts, L. Kecskes, L.J. Rowland, S.P. Mates, and S.D. Ridder, "Structure and Properties of Consolidated Amorphous Metal Powder," Powder Materials: Current Research and Industrial Practices III, edited by F.D.S. Marquis, Warrendale, PA: TMS, 2003.

\section{Other}

Basak, D., R.A. Overfelt, and D. Wang, "Measurement of specific heat capacity and electrical resistivity of industrial alloys using pulse heating techniques," International Journal of Thermophysics, 24 (6), 1721-1733, 2003.

Benderksy, L.A., I.D. Fawcett, and M. Greenblatt, "TEM study of two-dimensional incommensurate modulation in layered $\mathrm{La}_{2-2 \mathrm{x}} \mathrm{Ca}_{1+2 \mathrm{x}} \mathrm{Mn}_{2} \mathrm{O}_{7}$ $(0.6<\mathrm{x}<0.8)$," Chemical Materials, (submitted), 2004.

Bendersky, L.A., and I. Takeuchi, "Use of transmission electron microscopy in combinatorial studies of functional oxides," Macromolecular Rapid Comm., 25 (6), 695, 2004.

Bobordis, K., A. Seifter, A.W. Obst, and D. Basak, "Radiance temperatures and normal spectral emittances (in the wavelength range of $1500 \mathrm{~nm}$ to $5000 \mathrm{~nm}$ ) of tin, zinc, aluminum, and silver at their melting points by a pulse-heating technique," International Journal of Thermophysics, (in press), 2003.

Bobordis, K., A. Seifter, A.W. Obst, and D. Basak, "Validation of a new high-speed fiber-coupled four-wavelength infrared pyrometer in the range of $505 \mathrm{k}$ to $1234 \mathrm{k}$ by a pulse-heating technique, using the melting points of tin, zinc, aluminum, and silver as reference points," International Journal of Thermophysics, (in press), 2003. 
Boettinger, W.J., C.E. Campbell, T. Hansen, P. Merewether, and B.A. Mueller, "Properties of complex inorganic solids," edited by P.E.A. Turchi, A. Gonis, A. Meike, and K. Ragan, New York: Kluwer Academics/Plenum Publishers, (in press), 2003.

Boettinger, W.J., D. Josell, S.R. Coriell, and D. Basak, "Rapid melting of $\mathrm{Nb}-47$ mass \% Ti: effect of heating rate and grain size," Solidification Processes and Microstructures, edited by Rappaz, Beckermann and Trivedi, Warrendale, PA: TMS, pp. 87-98, 2004.

Chang, K.-S., M. Aronova, C.-L. Lin, M. Murakami, M.H. Yu, J. Hattrick-Simpers, O.O Famodu, S.Y. Lee, R. Ramesh, M. Wuttig, I. Takeuchi, C. Gao, and L.A. Bendersky, "Exploration of artificial multiferroic thin-film heterostructures using compositon spreads," Applied Physical Letters, 84 (16), 3091, 2004.

Famodu, O.O., J. Hattrick-Simpers, M. Aronova, K.-S. Chang, M. Murakami, M. Wuttig, T. Okazaki, Y.Furuya, L. Knauss, L.A. Bendersky, F.S. Biancaniello, and I. Takeuchi, "Combinatorial investigation of ferromagnetic shape-memory alloys in the Ni-Mn-Al ternary system suing a composition spread technique," Materials Transactions, 45, 173, 2004.

Kramer, D.E., M.F. Savage, A. Lin, and T.J. Foecke, "Novel method for TEM characterization of deformation under nanoindents in nanolayered materials," Scripta Materialia, 50 (6), 745-749, 2004.

Lu, C.J., L.A. Bendersky, K. Chang, and I. Takeuchi, "HRTEM study on the extended defect structure of epitaxial $\mathrm{Ba}_{0.3} \mathrm{Sr}_{0.7} \mathrm{TiO}_{3}$ thin films grown on (001) LaAl03," Proceedings of MRS Symposium, Vol. 751, 23.3, 2003.

Mates, S.P., F.S. Biancaniello, and S.D. Ridder, "NIST experiments in gas atomization 1986-1999," Proceedings of the 2004 International Conference on Powder Metallurgy and Particulate Materials, MPIF, Princeton, NJ, (submitted), 2004.

Mates, S.P., F.S. Biancaniello, and S.D. Ridder, "The effect of swirl on gas velocity decay in a generic annular close-coupled nozzle," Powder Materials: Current Research and Industries Practices III, edited by F.D.S. Marquis, Warrendale, PA: TMS, 2003.
Mates, S.P., and G.S. Settles, "A study of liquid metal atomization using close-coupled nozzles, part 1: gas dynamics," Atomization and Sprays, (in press), 2003.

Mates, S.P., and G.S. Settles, "A study of liquid metal atomization using close-coupled nozzles, part 2: atomization behavior," Atomization and Sprays, (in press), 2003.

Ricker, R.E., "Modeling the influence of crack path deviations on the propagation of stress corrosion cracks," Hydrogen Effects on Materials Behavior and Corrosion Deformation Interactions, edited by N.R. Moody, A.W. Thompson, R.E. Ricker, G.S. Was, and R.H. Jones, Warrendale, PA: TMS, pp. 629-638, 2003.

Ricker, R.E., and A.K. Vasudevan, "The influence of grain boundary precipitation on the stress corrosion cracking of Al-Li and Al-Li-Cu alloys," Hydrogen Effects on Materials Behavior and Corrosion Deformation Interactions, edited by N.R. Moody, A.W. Thompson, R.E. Ricker, G.S. Was, and R.H. Jones, Warrendale, PA: TMS, pp. 927-935, 2003.

Stoudt, M.R., and R.E. Ricker, "Electrochemical evaluation of a corrosion fatigue failure mechanism in a duplex stainless steel," Metallurgical and Materials Transactions A 35 (A) (8), 2427-2437, 2004.

Takeuchi, I., W. Yang, K.-S. Chang, M. Aronova, T. Venkatasan, R.D. Vespute, and L.A. Bendersky, "Monolithic multi-channel UV detector arrays and continuous phase evolution in $\mathrm{Mg}_{\mathrm{x}} \mathrm{Zn}_{1-\mathrm{x}} \mathrm{O}$ composition spreads," Journal of Applied Physics 94, 7736, 2003.

Tsuda, T., C.L. Hussey, and G.R. Stafford, Electrodeposition of Al-Mo Alloys from the lewis acidic aluminum chloride-1 ethyl-3-methylimidazolium chloride molten salt," Journal of Electrochemical Society, Vol. 151, Issue 8, pp. 379, 2004.

Tsuda, T., C.L. Hussey, G.R. Stafford, and O. Kongstein, "Electrodeposition of Al-Zr alloys from the lewis acidic aluminum chloride-1 ethyl-3methylimidazolium chloride melt," Journal of Electrochemical Society, 151, C445, 2004. 



\section{Metallurgy Division}

\section{Chief}

Carol A. Handwerker

Phone: 301-975-6158

E-mail: carol.handwerker@nist.gov

\section{Deputy Chief}

Frank W. Gayle

Phone: 301-975-6161

E-mail: frank.gayle@nist.gov

\section{NIST Fellows}

William J. Boettinger

Phone: 301-975-6160

E-mail: william.boettinger@nist.gov

\section{John W. Cahn}

Phone: 301-975-5664

E-mail: john.cahn@nist.gov

\section{Group Leaders}

Electrochemical Processing Group

Gery R. Stafford

Phone: 301-975-6412

E-mail: gery.stafford@nist.gov

Magnetic Materials Group

Robert D. Shull

Phone: 301-975-6035

E-mail: robert.shull@nist.gov

Materials Performance Group

Stephen D. Ridder

Phone: 301-975-6175

E-mail: stephen.ridder@nist.gov

Materials Structure and Characterization Group Frank W. Gayle

Phone: 301-975-6161

E-mail: frank.gayle@nist.gov

Metallurgical Processing Group

Stephen D. Ridder

Phone: 301-975-6175

E-mail: stephen.ridder@nist.gov 


\section{Research Staff}

Banovic, Stephen W.

stephen.banovic@nost.gov

Metal forming

Mechanical properties

Texture analysis

Beauchamp, Carlos R.

carlos.beauchamp@nist.gov

Standard reference materials

Bendersky, Leonid A.

leonid.bendersky@nist.gov

Functional ceramics

Analytical and high-resolution TEM

Metal glasses

Combinatorial methods

Biancaniello, Frank S.

frank.biancaniello@nist.gov

Spray deposition measurements and diagnostics

Inert gas atomization: metal powder

measurements and consolidation

Nitrogenated steels, standard reference materials

Special alloys, bulk metallic glasses

Biomaterials

\section{Boettinger, William J.}

william.boettinger@nist.gov

Relation of alloy microstructures to processing conditions; solidification

Thermal analysis

Solder

Bonevich, John E.

john.bonevich@nist.gov

Electron holography

Interfacial structure and chemistry

High resolution/analytical electron microscopy

Magnetic materials

\section{Campbell, Carelyn E.}

carelyn.campbell@nist.gov

Transient liquid phase bonding

Multicomponent diffusions simulations

Alloy design methodology

\section{Claggett, Sandra W.}

sandra.claggett@nist.gov

Specimen preparation for electron microscopy

Digital imaging

Specimen preparation for scanning electron microscopy

Image analysis

Metallography of materials
Drew, Rosetta V.

rossetta.drew@nist.gov

Magnetometry

Database maintenance

Egelhoff Jr., William F. william.egelhoff@nist.gov

Magnetic thin films

Ultrasoft magnets

Magnetic sensors

Ultrahigh density data storage media

Fields, Richard J.

richard.fields@nist.gov

Mechanical properties

Mechanical testing

Powder consolidation

Metal forming

Fink, James L.

james.fink@nist.gov

Hardness standards

Hardness testing

Mechanical properties measurement

Metal powder consolidation

Environmental corrosion

Metallography

Foecke, Timothy J.

timothy.foecke@nist.gov

Metal forming standards

Nanostructured materials

Experimental fracture physics

SEM, TEM, SPM

Micro- and nano-mechanics of materials

Dislocation-based deformation mechanisms

Historical metallurgy and failure analysis

Gates, Hilary G.

hilary.gates@nist.gov

Standard reference materials

Gayle, Frank W.

frank.gayle@nist.gov

Structure/property relationships

Transmission electron microscopy

Intrastructural materials

Guyer, Jonathan E. jonathan.guyer@nist.gov

III-V semiconductors

Molecular beam epitaxy

Alloy film deposition

Stress-induced mass transport 


\section{Handwerker, Carol A.}

carol.handwerker@nist.gov

Interface thermodynamics and kinetics

Solder science

Microstructure evolution

Iadicola, Mark

mark.iadicola@nist.gov

Mechanical properties

Mechanical testing

Stress measurement

Metal forming

Shape memory alloys

\section{Jiggetts, Rodney D.}

rodney.jiggetts@nist.gov

Substrate analysis

Grit blasting

Roughness measurements

Metallography of coatings

Image analysis

Micro analysis/characterization

Hot isostatic processing

Johnson, Christian E.

christian.johnson@nist.gov

Ultra-black coatings

Electroless deposition processes

Metallic glass alloy deposition

Microhardness SRM research

Chromium deposition

Pulse alloy deposition

Lead-free solder

\section{Johnson, Frank}

frank.johnson@nist.gov

Soft magnetic materials

Nanostructured metallic alloys

Magnetocaloric materials

Magnetic properties measurements

Metallographic analysis

Josell, Daniel

daniel.josell@nist.gov

Mechanical and structural properties of multilayer materials

Electrical properties of submicrometer interconnects

Filling of sub-100 $\mathrm{nm}$ features by electrodeposition

\section{Kattner, Ursula R.}

ursula.kattner@nist.gov

Computational thermodynamics

Alloy phase equilibria evaluations

Solder alloy systems

Superalloy systems

Metal hydrogen systems
Kelley, David R.

david.kelley@nist.gov

Microhardness SRM development

Dye penetration SRM development

Precious metal electrodeposition

Plating on aluminum

Electroplating

Levine, Lyle E.

lyle.levine@nist.gov

Diffraction theory

Dislocation-based deformation

Synchrotron $\mathrm{x}$-ray techniques

Percolation theory

TEM, SEM, SPM

Kolsky bar techniques

Nanomechanics

Multiscale modeling

Low II, Samuel R.

samuel.low@nist.gov

Hardness standards

Hardness testing

Mechanical properties of materials

Mechanical testing of material

Luecke, William E.

william.luecke@nist.gov

High-temperature mechanical testing

Fire-resistant steel

Creep of ceramics

Silicon nitride

Maranville, Brian B.

brian.maranville@nist.gov

Ferromagnetic resonance

Perpendicular magnetic media

Atomic force microscopy

Mates, Steven P.

steven.mates@nist.gov

Compressible fluid flow

Metal sprays

Fluid flow visualization

McMichael, Robert D.

robert.mcmichael@nist.gov

Giant magnetoresistance

Ferromagnetic resonance

Nanocomposites

Moffat, Thomas P

thomas.moffat@nist.gov

Electrochemistry

Scanning probe microscopy

Nanostructures

Surfactant mediated growth (ADD) 
Pierce Jr., Thomas A. tpierce@nist.gov

Hardness measurements

Metallography

Pitchure, David J.

david.pitchure@nist.gov

Mechanical testing

Computer-aided design

Mechanical engineering

Rantschler, James O.

rantschl@nist.gov

Ferromagnetic resonance

Magnetic characterization

Damping formalisms

Ricker, Richard E.

richard.ricker@nist.gov

Corrosion mechanisms

Deformation mechanisms

Stress corrosion cracking (SCC)

Hydrogen embrittlement

Corrosion fatigue

Dynamic mechanical analysis

\section{Ridder, Stephen D.}

stephen.ridder@nist.gov

Metal powder production and characterization

Metal spray processing

Process metallurgy, modeling, and control

\section{Shapiro, Alexander J.}

alexander.shapiro@nist.gov

Magnetic domain imaging

Mossbauer effect

Scanning electron microscopy (SEM) and

$\mathrm{X}$-ray microanalysis

Image analysis
Shull, Robert D.

robert.shull@nist.gov

Nanocomposites

Magnetic susceptibility

Mossbauer effect

$\mathrm{X}$-ray and neutron diffraction

Magneto-caloric and magneto-optical effects

Magnetic domain imaging

Stafford, Gery R.

gery.stafford@nist.gov

Electrochemical transients

Electrodeposition

Molten salt electrochemistry

Stress in thin films (ADD)

Stoudt, Mark R.

mark.stoudt@nist.gov

Deformation-induced surface roughness

Mechanical properties

Metal fatigue

Thin films

Environmentally assisted cracking

Warren, James A.

james.warren@nist.gov

Computer simulations of solidification

Dendrite pattern formation

Modeling of solder/substrate wetting processes

Modeling grain boundaries

Williams, Maureen E.

maureen.williams@nist.gov

Differential thermal analysis

Powder x-ray diffraction

Solder Wettability

SEM 


\section{Organizational Charts}

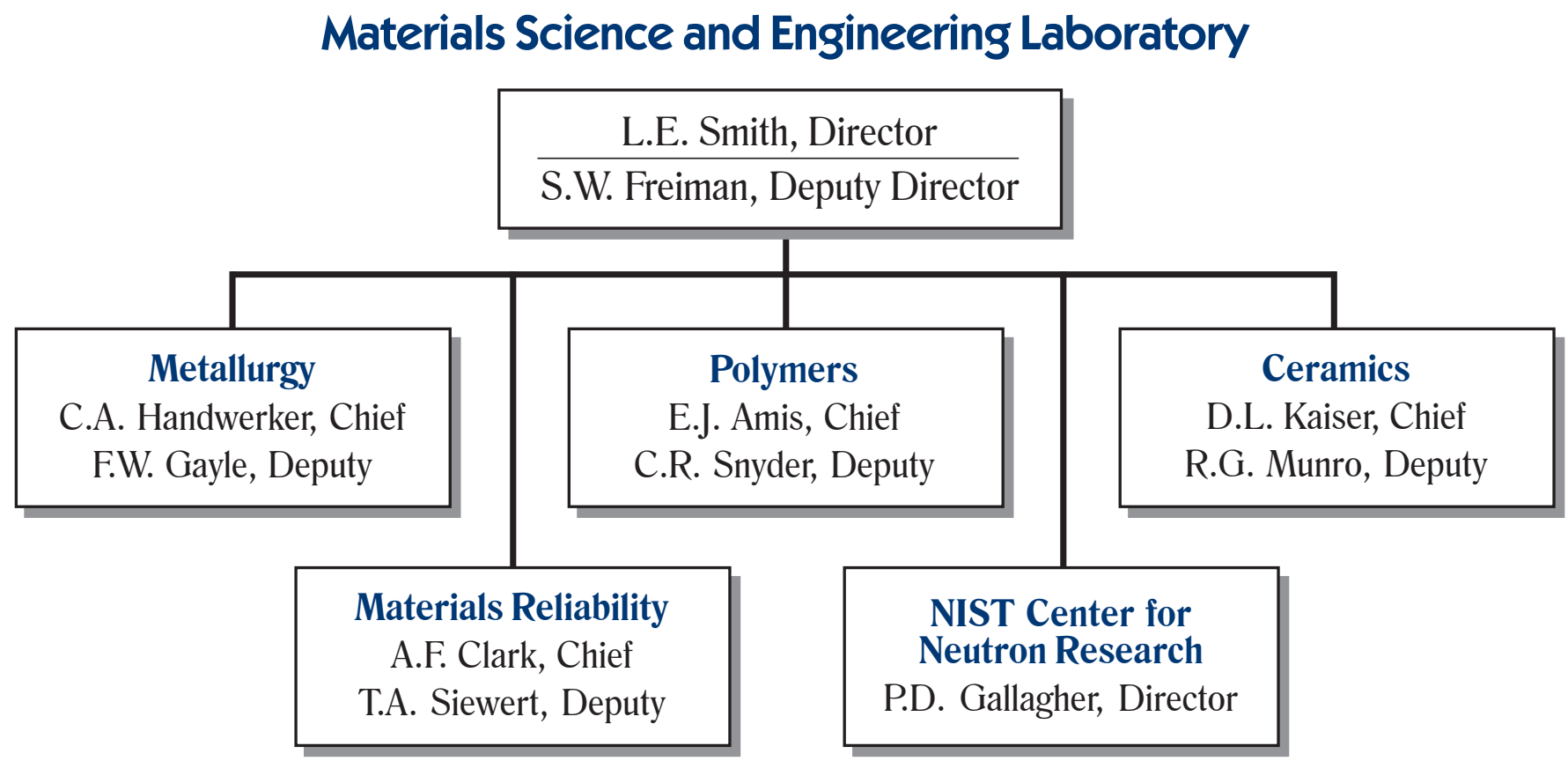

National Institute of Standards and Technology

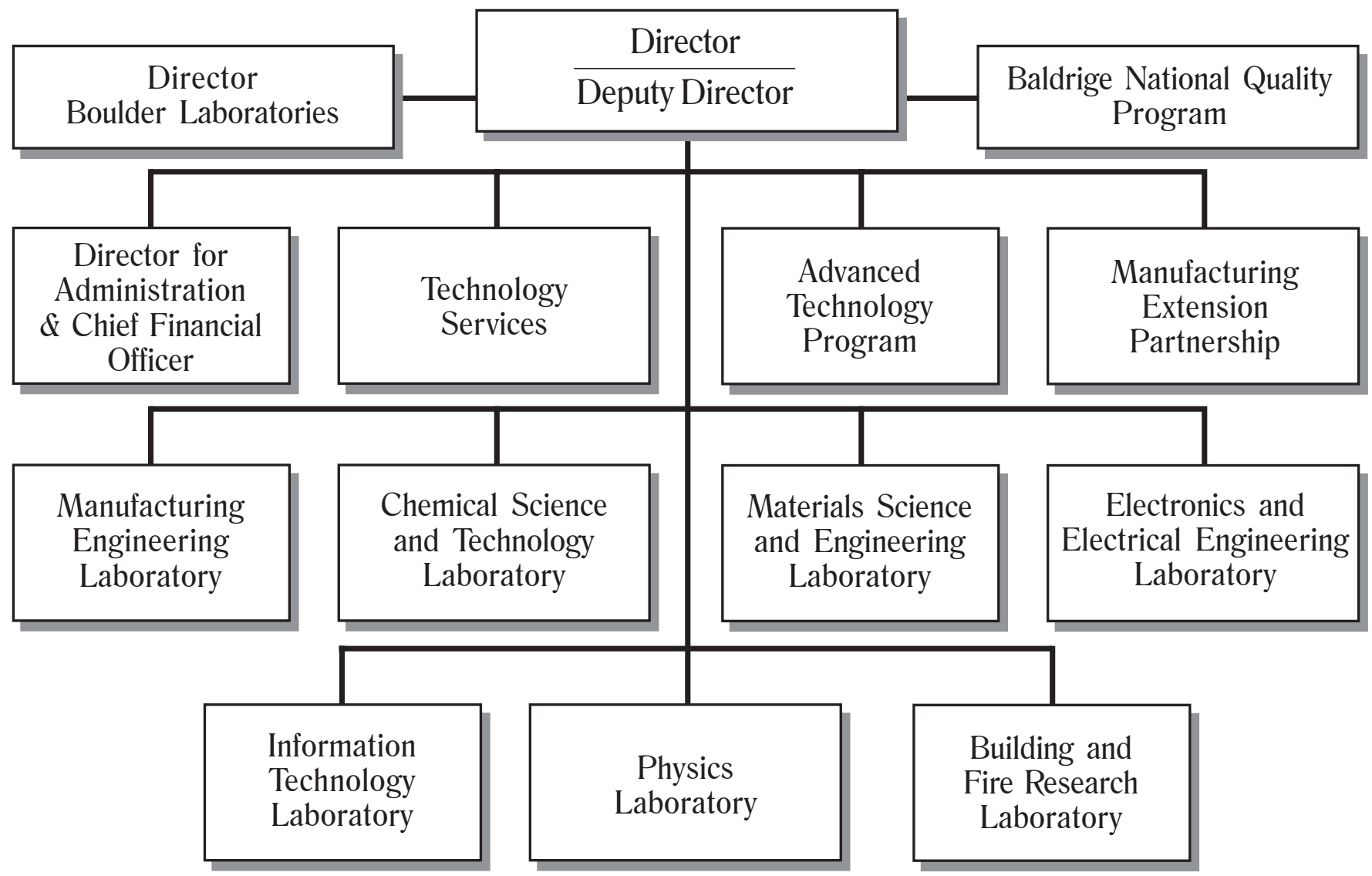




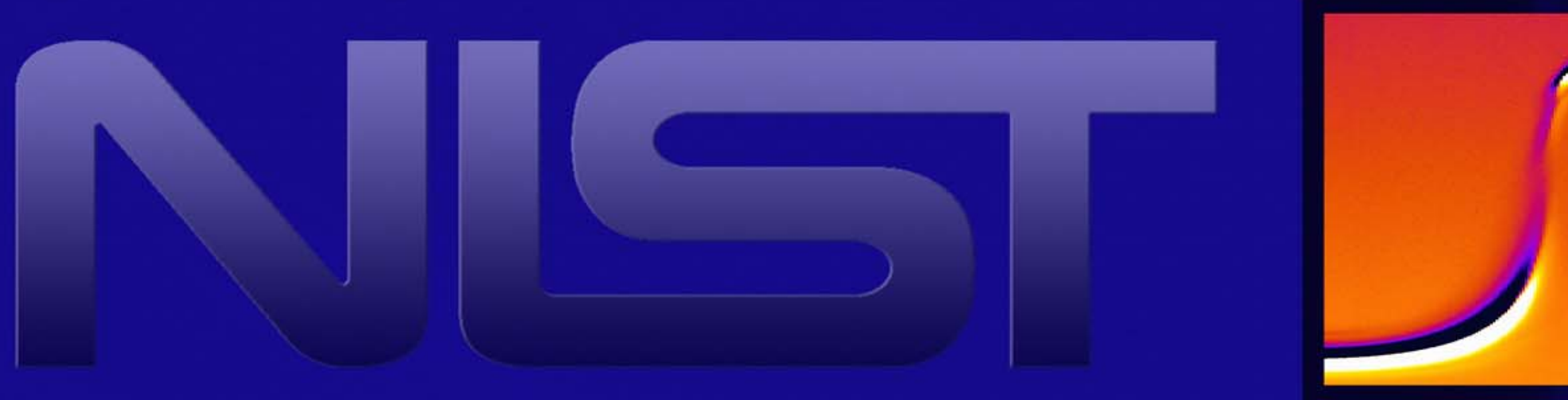